\title{
Multimodal Noncontact Tracking of Surgical Instruments
}

Tara Bracken, The University of Western Ontario

Supervisor: Dr. Michael Naish, The University of Western Ontario

A thesis submitted in partial fulfillment of the requirements for the Master of Engineering

Science degree in Mechanical and Materials Engineering

(C) Tara Bracken 2013

Follow this and additional works at: https://ir.lib.uwo.ca/etd

Part of the Electro-Mechanical Systems Commons

\section{Recommended Citation}

Bracken, Tara, "Multimodal Noncontact Tracking of Surgical Instruments" (2013). Electronic Thesis and Dissertation Repository. 1558.

https://ir.lib.uwo.ca/etd/1558

This Dissertation/Thesis is brought to you for free and open access by Scholarship@Western. It has been accepted for inclusion in Electronic Thesis and Dissertation Repository by an authorized administrator of Scholarship@Western. For more information, please contact wlswadmin@uwo.ca. 


\title{
Multimodal Noncontact Tracking of Surgical Instruments
}

(Thesis Format: Monograph)

by

\section{Tara Bracken}

\author{
Faculty of Engineering \\ Department of Mechanical and Materials Engineering
}

Submitted in partial fulfillment

of the requirements for the degree of

Master of Engineering Science

School of Graduate and Postdoctoral Studies

The University of Western Ontario

London, Ontario, Canada

(C) Tara Bracken, 2013 


\title{
Multimodal Noncontact Tracking of Surgical Instruments
}

\author{
Tara Bracken \\ M.E.Sc. Thesis, 2013 \\ Department of Mechanical and Materials Engineering \\ The University of Western Ontario
}

\begin{abstract}
For many procedures, open surgery is being replaced with minimally invasive surgical (MIS) techniques. The advantages of MIS include reduced operative trauma and fewer complications leading to faster patient recovery, better cosmetic results and shorter hospital stays.

As the demand for MIS procedures increases, effective surgical training tools must be developed to improve procedure efficiency and patient safety. Motion tracking of laparoscopic instruments can provide objective skills assessment for novices and experienced users. The most common approaches to noncontact motion capture are optical and electromagnetic (EM) tracking systems, though each approach has operational limitations. Optical trackers are prone to occlusion and the performance of EM trackers degrades in the presence of magnetic and ferromagnetic material. The cost of these systems also limits their availability for surgical training and clinical environments.

This thesis describes the development and validation of a novel, noncontact laparoscopic tracking system as an inexpensive alternative to current technology. This system is based on the fusion of inertial, magnetic and distance sensing to generate real-time, 6-DOF pose data. Orientation is estimated using a Kalman-filtered attitude-heading reference system (AHRS) and restricted motion at the trocar provides a datum from which position information can be recovered.

The Inertial and Range-Enhanced Surgical (IRES) Tracker was prototyped, then validated using a MIS training box and by comparison to an EM tracking system. Results of IRES tracker testing showed similar performance to an EM tracker with position error as low as $1.25 \mathrm{~mm}$ RMS and orientation error $<0.58^{\circ}$ RMS along each axis. The IRES tracker also displayed greater precision and superior magnetic interference rejection capabilities. At a fraction of the cost of current laparoscopic tracking methods, the IRES tracking system would provide an excellent alternative for use in surgical training and skills assessment.
\end{abstract}

Keywords: minimally invasive surgery (MIS), laparoscopic, 6-DOF instrument tracking, position and orientation, inertial measurement unit (IMU), attitude-heading reference system (AHRS) 


\section{Contents}

$\begin{array}{ll}\text { Abstract } & \text { ii }\end{array}$

$\begin{array}{ll}\text { Table of Contents } & \text { iii }\end{array}$

List of Figures

List of Tables $\quad$ viii

Nomenclature and Acronyms $\quad$ ix

1 Introduction $\quad 1$

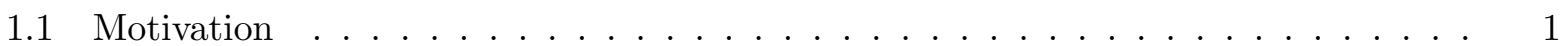

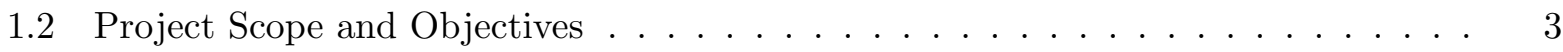

1.3 Overview of the Thesis $\ldots \ldots \ldots \ldots \ldots \ldots \ldots \ldots$

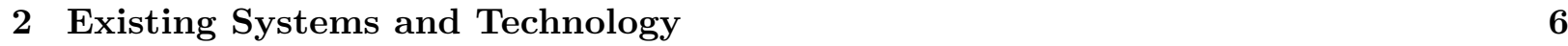

2.1 Mechanical Tracking Systems . . . . . . . . . . . . . . . . . . . . . 6

2.2 Electromagnetic $(\mathrm{EM})$ Tracking Systems . . . . . . . . . . . . . 8

2.3 Optical Tracking Systems _. . . . . . . . . . . . . . . . . . . . . . . 10

2.3 .1 SIMIS Vision Tracker f . . . . . . . . . . . . . . . . . . 11

2.4 Alternate Approaches to Instrument Tracking . . . . . . . . . . . . . . . 12

2.5 MEMS Technology . . . . . . . . . . . . . . . . . . . . . 13

2.6 Inertial Measurement Units $(\mathrm{IMU}) \ldots \ldots \ldots \ldots \ldots$

2.6.1 Inertial Navigation Systems (INS) f . . . . . . . . . . . . . . 14 
2.6.2 Attitude-Heading Reference Systems (AHRS) . . . . . . . . . . . . 16

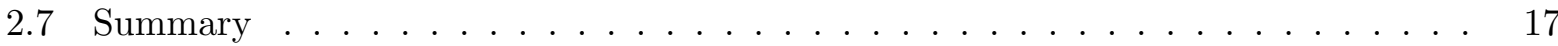

3 System Design $\quad 19$

3.1 Coordinate Frames and Conventions . . . . . . . . . . . . . . . . . . . . 19

3.2 Hardware System Design . . . . . . . . . . . . . . . . . . . . . . . . . 20

3.2 .1 INS Sensors . . . . . . . . . . . . . . . . . . . . . 21

3.2.1.1 Sensor Selection Case Study _. . . . . . . . . . . . . . . . 21

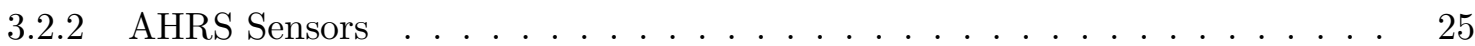

3.2 .2 .1 Phidgets 9 -DOF IMU . . . . . . . . . . . . . . . 28

3.2 .3 Hardware Configuration . . . . . . . . . . . . . . . . . . . . . . 30

3.2.3.1 Sharp IR Distance Sensor . . . . . . . . . . . . . . . . . . . . . . 31

3.3 Algorithm Development . . . . . . . . . . . . . . . . . . . 32

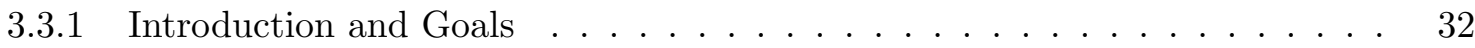

3.3 .2 Euler Angles and Quaternions _. . . . . . . . . . . . . . . . 34

3.3 .3 AHRS Sensor Fusion Algorithms . . . . . . . . . . . . . . . . . . 37

3.3.3.1 Complementary Filter . . . . . . . . . . . . . . 37

3.3.3.2 Linear Kalman Filter . . . . . . . . . . . . . . . . . . . 38

3.3.3.3 Model Validation . . . . . . . . . . . . . . . . . . . . . 41

3.3.3.4 Nonlinear Kalman Filter . . . . . . . . . . . . . . . . . . . . . . 41

3.3.4 A Quaternion-Based Linear Kalman Filter for Orientation Sensing . . . . . 44

3.3.5 Position Tracking . . . . . . . . . . . . . . . . . . . . . 48

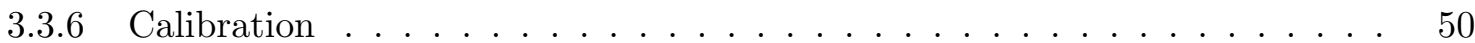

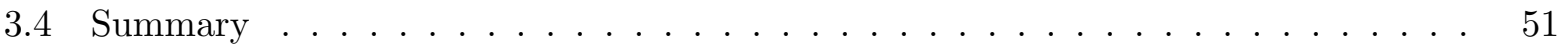

4 Testing and Validation $\quad 52$

4.1 Testing Methodology . . . . . . . . . . . . . . . . . . 52

4.1 .1 System Registration and Calibration . . . . . . . . . . . . . . . . . . 53

4.1 .2 Testing Procedure $\ldots \ldots \ldots \ldots \ldots \ldots \ldots$

4.1 .2 .1 Accuracy . . . . . . . . . . . . . . . 57 
4.1.2.2 Orientation Accuracy . . . . . . . . . . . . . . . 58

4.1 .2 .3 Precision . . . . . . . . . . . . . . . . 59

4.1.2.4 Repeatability . . . . . . . . . . . . . . . . . 59

4.1 .2 .5 Data Spread . . . . . . . . . . . . . . . . . 60

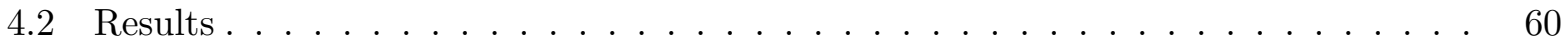

4.2 .1 Discussion . . . . . . . . . . . . . . . . . . . 60

4.2.1.1 Accuracy . . . . . . . . . . . . . . . . . 60

4.2.1.2 Orientation Accuracy . . . . . . . . . . . . . . 65

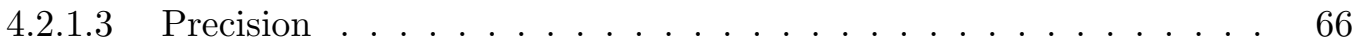

4.2.1.4 Repeatability ..................... 67

4.2.1.5 95th and 99th Percentile . . . . . . . . . . . . 68

4.2.1.6 Computational Performance _. . . . . . . . . . . . . 69

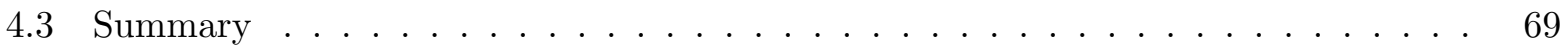

5 Conclusions and Recommendations $\quad 71$

5.1 Summary and Conclusions . . . . . . . . . . . . . . . . . . . . . . . 72

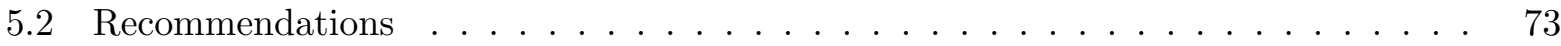

$\begin{array}{ll}\text { References } & \mathbf{7 6}\end{array}$

$\begin{array}{lr}\text { Vita } & 80\end{array}$ 


\section{List of Figures}

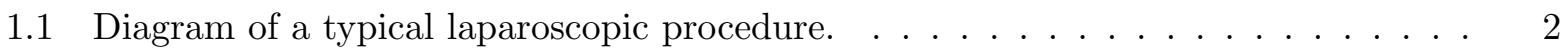

2.1 Diagram of a mechanical arm linking a reference location with target location. . . 7

2.2 Diagram of an electromagnetic tracking system with field generator and sensor probe. 9

2.3 Triangulation method for an optical tracking system. . . . . . . . . . . . 11

2.4 Image segmentation and analysis of a laparoscopic camera frame to recover 6-DOF pose information for the SIMIS vision tracker. . . . . . . . . . . . . . 12

$3.1 \mathrm{~N}, \mathrm{~L}$ and B coordinate frame conventions. . . . . . . . . . . . . . . 20

3.2 Movement of a laparoscopic instrument across the tissue layer. . . . . . . . . . . 30

3.3 Sharp GP2D120 distance to voltage output characteristics. . . . . . . . . . . . . 32

3.4 Laparoscopic instrument augmented with the IRES Tracker. . . . . . . . . . . . . . 33

3.5 Laparoscopic instrument operating in a training box. . . . . . . . . . . . . . . 33

3.6 Right-handed coordinate system defining Euler angles. . . . . . . . . . . . . 35

3.7 Four quaternion parameters representing orientation in 3 -space. . . . . . . . . . . . 36

3.8 Visual representation of the extended and unscented Kalman filters. . . . . . . . . 42

3.9 Diagram of system length variables for dimensional analysis. . . . . . . . . . . . 49

4.1 Instrument tip and calibration grid inside of the training box. . . . . . . . . . . . 54

4.2 Sample image captured from the laparoscopic camera during a 20-point test. . . . 54

4.3 Magnetometer calibration for hard and soft iron field distortions. . . . . . . . . . 56

4.4 Raw IRES tracker position information (left) and registered IRES tracker position

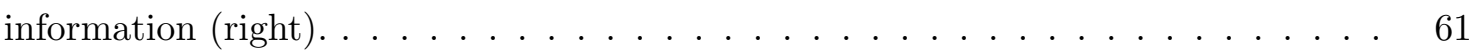


4.5 IRES tracker position estimates for a typical 20-point volume. . . . . . . . . . . 63

4.6 Electromagnetic tracker position estimates for a typical 20-point volume. . . . . . . 63

4.7 IRES tracker position estimates for a 60 -point volume. . . . . . . . . . . . . . . . 64

4.8 Electromagnetic tracker position estimates for a 60 -point volume. . . . . . . . . . 64

4.9 IRES tracker position estimates for a 20-point volume with time-varying electro-

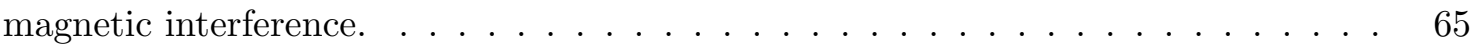




\section{List of Tables}

2.1 Mechanical Arm Tracking Systems. . . . . . . . . . . . . . . . . . . 8

2.2 Comparison of three electromagnetic tracking systems. . . . . . . . . . . . 9

2.3 Comparison of optical tracking systems with similar fields of measurement (FOM). 11

2.4 Accelerometer bias error expressed in $\mathrm{mg}\left(9.81 \times 10^{-3} \mathrm{~m} / \mathrm{s}^{2}\right) \ldots \ldots \ldots$

2.5 Accelerometer misalignment error. . . . . . . . . . . . . . . 15

2.6 Gyro angle random walk error. . . . . . . . . . . . . . . 16

3.1 Examples of commercially-available MEMS-based INS solutions. . . . . . . . . . . 24

3.2 Static error due to accelerometer bias. . . . . . . . . . . . . . . . 26

3.3 Static error due to accelerometer misalignment. . . . . . . . . . . . . . . . 26

3.4 Phidgets Spatial 1056 9-DOF IMU Specifications. . . . . . . . . . . . . . . . . 29

4.1 Summary of Results. . . . . . . . . . . . . . . . . . . . . . . . . . . . 61

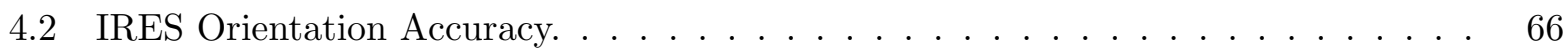

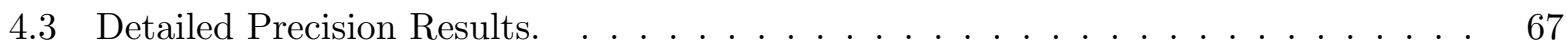

4.4 Computational Requirements for the MATLAB-based IRES Tracker. . . . . . . . . 69 


\section{Nomenclature and Acronyms}

\section{Latin Letters}

g Gravitational acceleration approximated as $9.81 \frac{\mathrm{m}}{\mathrm{s}^{2}}$

$g_{\mathrm{x}}, g_{\mathrm{y}}, g_{\mathrm{z}} \quad$ Gravitational acceleration measured along each axis of the accelerometer

F Transition state model

H Observation model

$h_{\mathrm{x}}, h_{\mathrm{y}} \quad$ Components of horizontal heading (yaw) angle

K Kalman gain

$l_{\mathrm{di} \mid \mathrm{rp}} \quad$ Distance between the reflective trocar disk and the trocar pivot point

$l_{\mathrm{IR} \mid \mathrm{di}} \quad$ Distance between the IR sensor and reflective trocar disk

$l_{\mathrm{IR} \mid \mathrm{ti}} \quad$ Length of the laparoscopic instrument from IR sensor to tip

$m_{\mathrm{x}}, m_{\mathrm{y}}, m_{\mathrm{z}} \quad$ Magnetic field readings along each axis of the magnetometer

$\mathbf{P}_{t \mid t-1} \quad$ Predicted (a priori) covariance matrix

$\mathbf{P}_{t \mid t} \quad$ Updated (a posteriori) covariance matrix

Q Process noise covariance matrix

$q_{1}, q_{2}, q_{3}, q_{4} \quad$ Components of a quaternion

R Observation noise covariance matrix

$\vec{w}_{\mathrm{x}}, \vec{w}_{\mathrm{y}}, \vec{w}_{\mathrm{z}} \quad$ Components of process noise

$w_{\mathrm{R}} \quad$ Observation covariance tuning parameter

$\vec{x}_{t \mid t \quad \quad \text { A posteriori } \text { state estimate }}$ 


$\begin{array}{ll}\vec{x}_{t \mid t-1} & \text { A priori state estimate } \\ \vec{x}_{t-1 \mid t-1} & \text { State estimate from previous iteration } \\ \vec{z}_{t \mid t} & \text { Observation vector }\end{array}$

\section{Greek Letters}

$\begin{array}{ll}\mu & \text { Micro }\left(10^{-6}\right) \\ \omega & \text { Angular velocity } \\ \phi & \text { Pitch Euler angle } \\ \pi & \mathrm{Pi} \\ \psi & \text { Yaw Euler angle } \\ \sigma & \text { Standard Deviation } \\ \sigma^{2} & \text { Variance } \\ \theta & \text { Roll Euler angle }\end{array}$

\section{Acronyms}

$\begin{array}{ll}\text { 2-D } & \text { Two-Dimensional } \\ 3 \text {-D } & \text { Three-Dimensional } \\ \text { ADC } & \text { Analog to Digital Converter } \\ \text { AHRS } & \text { Attitude Heading Reference System } \\ \text { CAD } & \text { Canadian Dollars } \\ \text { CMOS } & \text { Complementary Metal-Oxide-Semiconductor } \\ \text { CPU } & \text { Central Processing Unit } \\ \text { CSTAR } & \text { Canadian Surgical Technologies and Advanced Robotics } \\ \text { DOF } & \text { Degrees of Freedom } \\ \text { EKF } & \text { Extended Kalman Filter } \\ \text { EM } & \text { Electromagnetic }\end{array}$




\begin{tabular}{|c|c|}
\hline FOM & Field of Measurement \\
\hline FOV & Field Of View \\
\hline GPS & Global Positioning System \\
\hline IMU & Inertial Measurement Unit \\
\hline INS & Inertial Navigation System \\
\hline IR & Infrared \\
\hline IRES & Inertial and Range Enhanced Surgical \\
\hline $\mathrm{KF}$ & Kalman Filter \\
\hline LED & Light-Emitting Diode \\
\hline LKF & Linear Kalman Filter \\
\hline LS & Least-Squares \\
\hline LSB & Least Significant Bit \\
\hline MEMS & Microelectromechanical Systems \\
\hline MIS & Minimally Invasive Surgery \\
\hline MRI & Magnetic Resonance Imaging \\
\hline NED & North East Down \\
\hline $\mathrm{PC}$ & Personal Computer \\
\hline PPM & Parts Per Million \\
\hline QUEST & QUaternion ESTimator \\
\hline $\mathrm{RCM}$ & Remote Centre of Motion \\
\hline RMS & Root Mean Square \\
\hline RMSE & Root Mean Square Error \\
\hline SIMIS & Sensorized Instrument-based Minimally Invasive Surgical \\
\hline SPS & Samples Per Second \\
\hline UKF & Unscented Kalman Filter \\
\hline USB & Universal Serial Bus \\
\hline
\end{tabular}


USD United States Dollars 


\section{Chapter 1}

\section{Introduction}

It is expected that over the next 5 years, the United States market for laparoscopic devices will display steady growth, reaching an estimated value of $\$ 3.59$ billion by 2017 [1]. The demand for minimally invasive surgical (MIS) procedures is growing at a faster rate than open surgeries with much of the growth focused on single-port procedures. Some of the main benefits of MIS techniques include reduced operative trauma allowing for fewer complications, faster patient recovery and better cosmetic results. Recent improvements in MIS instrumentation and related technologies have made minimally invasive techniques accessible to surgeons in a broader range of specialties.

The fastest growing laparoscopic procedure is bowel resection followed by sleeve gastrecomies and hernia repairs. Other areas of growth include high-volume, single-port procedures such as cholecystectomies and appendectomies [1]. The best way to continue driving the adoption of MIS techniques from a technological standpoint is by making instrumentation simpler, more versatile, more robust and cheaper. A recent Millennium Research Group report on the future of laparoscopic devices indicated that an emphasis on cost cutting and the adoption of reusable and versatile products will result in faster market growth, especially within the hand instrument segment [1].

\subsection{Motivation}

A very important component of laparoscopic procedures involves instrument tracking. When the laparoscopic instrument is passed through a port, the surgeon loses information about the position 
and orientation of the device and must rely on a laparoscopic camera to provide a visual reference as shown in Figure 1.1. In many cases, the use of these cameras requires a second incision, which complicates the procedure and does not guarantee a continuous instrument tracking solution. These cameras are prone to occlusion by tissues and fluids and since they operate within an independent frame of reference, their viewpoint often proves disorienting to the surgeon. This increases the risk of accidental damage to nearby tissue, and as a result, more experienced surgeons are opting to perform high-volume procedures through a single-port with the use of alternative instrument tracking technology [1].

Effective surgical training and skills assessment can also help to improve procedure safety and efficiency. A tracking system can be used to record instrument motion and provide quantitative data for an objective analysis of the user's performance. This analysis may include an assessment of instrument path length, accuracy, changes in direction and smoothness of motion [2]. By comparing the motion profile of an experienced surgeon with that of a novice, surgical training can become faster and more effective.

The most common noncontact laparoscopic tracking systems are based on optical and electromagnetic (EM) technology. While both of these approaches provide high quality pose information, limitations exist that restrict their usefulness. Optical tracking systems are prone to occlusion and can become unreliable if the line-of-sight between the stereoscopic cameras and fiducial markers is

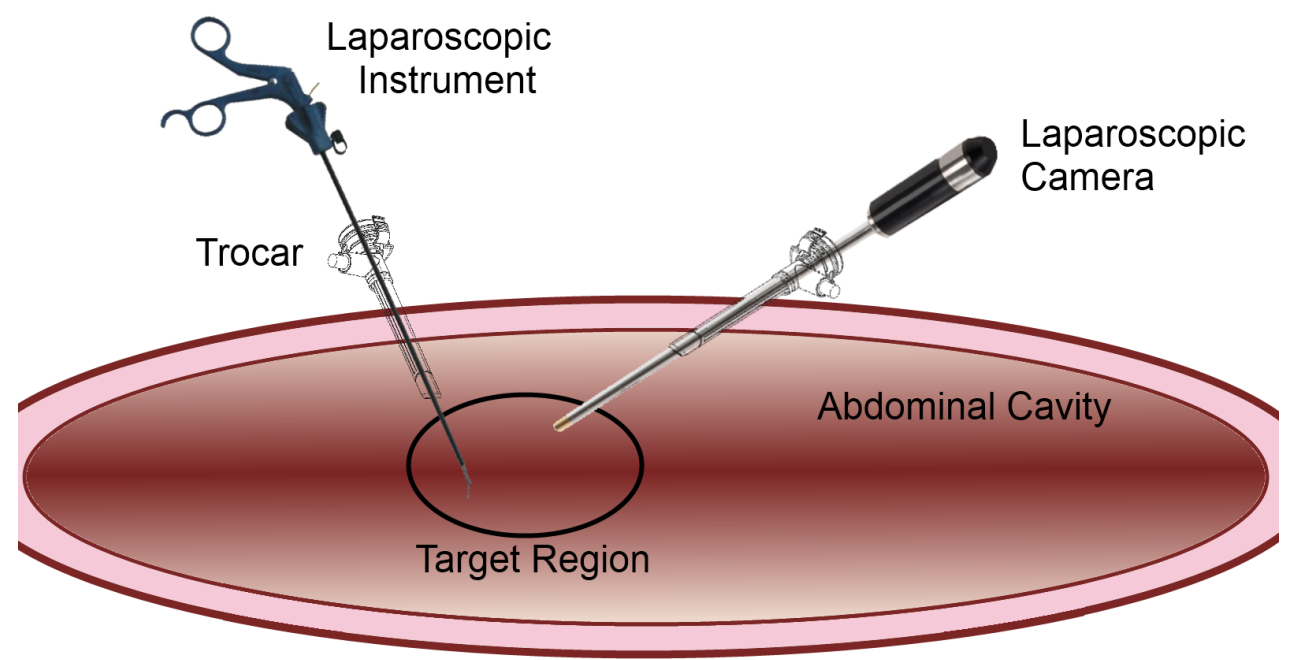

Figure 1.1: Diagram of a typical laparoscopic procedure. 
lost. EM tracking systems resolve position and/or orientation by sensing the strength and direction of an artificially generated magnetic field. These systems are not prone to occlusion; however, any magnetic fields or ferromagnetic material introduced into the operating environment will cause significant performance degradation. Additionally, both optical and EM technologies tend to be expensive, with costs that can be prohibitive for schools, clinics and hospitals.

Understanding that current tracking technologies are expensive and have limitations that restrict their versatility, the motivation behind this research is to explore alternative technologies and to develop an inexpensive, robust and versatile tracking solution. The goal is to improve accessibility to effective surgical training tools and to meet the growing demand within the laparoscopic device market.

\section{$1.2 \quad$ Project Scope and Objectives}

The demand for MIS procedures is expected to continue to grow for the foreseeable future and with that demand comes the need for effective surgical training and skills assessment. This thesis will investigate the current state of the art in medical device tracking and provide an analysis of the strengths and weaknesses of these approaches. By focusing on cost, versatility and robustness, existing technologies can be assessed to identify performance gaps that can be addressed with the development of a competing solution. Once the need for an alternate solution has been identified, specific project goals can be defined.

For the successful development of a new laparoscopic tracking solution, the following research objectives have been identified:

1. To design and develop a noncontact laparoscopic instrument tracking system based on the fusion of multiple sensing modalities.

2. To provide real-time 6 degree of freedom (DOF) tracking of instrument orientation and tip position. To ensure an acceptable level of performance, position accuracy should be comparable to modern EM tracking systems.

3. To demonstrate successful operation under conditions that cause alternative tracking systems 
to fail.

4. To develop a robust solution that can be easily adapted to a wide range of laparoscopic instruments.

5. To develop computational techniques and algorithms that are efficient enough to be implemented on embedded processors.

6. To provide an inexpensive approach to instrument tracking, thereby reducing the barrier to entry compared with standard medical tracking systems.

The most basic requirement for any laparoscopic tracking system is the ability to provide orientation and position information in real time in a format that can be easily interpreted. Noncontact tracking systems tend to be preferred by the user [3] because no physical connection between the system and the target device is required. This gives the surgeon full range of motion to plan an optimized procedure with minimal interference. These criteria must be maintained for any alternative tracking system.

That said, there are medical operating conditions under which the leading tracking systems cannot provide reliable pose information. It will be shown that through the fusion of existing sensing modalities, a new approach can be developed that provides extended sensing capability.

With the market push toward versatility, the developed solution should be adaptable to operate with a range of hand-held laparoscopic instruments. A tracking system that is inexpensive, robust and versatile will improve accessibility to the tools required for surgical training and skills assessment resulting in more capable surgeons, safer MIS procedures and improved patient care.

\subsection{Overview of the Thesis}

The structure of this thesis is summarized in the following outline:

Chapter 1: Introduction

This chapter presents the motivation behind this research, defines the project scope and outlines the research objectives. 
Chapter 2: Existing Systems and Technology

This chapter explores the capabilities and limitations of leading medical tracking technologies and investigates alternative approaches based on inertial sensing. Inertial Navigation Systems (INS) and Attitude-Heading Reference Systems (AHRS) are introduced to provide a foundation for the work conducted in Chapter 3.

\section{Chapter 3: System Design}

This chapter presents the hardware and software design and implementation of the Inertial and Range-Enhanced Surgical (IRES) tracking system. Hardware design includes sensor selection and the modification of a laparoscopic instrument to allow for the recovery of 6-DOF pose information. Software design explores methods for representing 3-D orientation and develops complementary and Kalman-based approaches to signal processing and sensor fusion. An implementation of the Linear Kalman Filter for AHRS sensor fusion is presented and augmented with distance information for real-time 6-DOF pose tracking.

\section{Chapter 4: Testing and Validation}

In this chapter, the IRES tracker is independently evaluated and compared with an EM tracking system. The testing methodology for both systems is presented with discussion of calibration procedures and the specific approaches used for data collection and analysis. All test results are presented and a thorough discussion of the system performance and capabilities is provided.

Chapter 5: Conclusions and Recommendations

This chapter concludes the thesis and provides a summary of the work and contributions made to the medical tracking community. Suggestions for improvement and recommendations for future work are also discussed. 


\section{Chapter 2}

\section{Existing Systems and Technology}

For much of the $20^{\text {th }}$ century, the only available approach to surgical localization and instrument tracking involved the use of mechanical jigs and stereotactic frames. These devices were often procedure-specific, cumbersome and unable to meet the needs of the surgeon, leading to new mechanical approaches being explored by the 1980s. Owing largely to advancements in computer and sensor technology, new generations of mechanical systems were able to provide a more general tracking solution with application to a broader range of surgical procedures.

Since the 1980s, a host of computer-aided tracking solutions have been developed including, but not limited to, mechanical, ultrasonic, fluoroscopic, optical and electromagnetic approaches. Of these, mechanical, optical and electromagnetic systems have become the most popular, with capabilities and limitations that make them uniquely suited to different types of procedures. In this chapter, these three technologies will be explored, the need for an alternate solution will be identified and the concept of inertial sensing using MEMS devices will be introduced to lay the foundation for the research and concepts presented in Chapters 3 and 4.

\subsection{Mechanical Tracking Systems}

The use of mechanical arms for surgical tracking was first introduced in the 1980s for the field of neurosurgery, where high localization accuracy was essential [4] [5]. Proving to be more robust and versatile than previous stereotactic approaches such as the Mayfield clamp, mechanical tracking 
systems sense orientation and position through direct mechanical linkages that connect a reference point with the target contact location, as illustrated in Figure 2.1. The mechanical arm contains rotary encoders within each joint to measure changes in position and orientation in the reference frame of the tracker. Mechanical tracking systems tend to exhibit very high accuracy and fast response times compared with noncontact tracking methods. A forward kinematic model based on the system geometry can be applied to achieve very fast refresh rates in the range of thousands of points per second. Despite these advantages, the use of mechanical tracking systems remains limited due to restrictions imposed on the user's range of motion, the inability to track multiple devices simultaneously and the difficulty of sterilizing the equipment for use in an operating room environment.

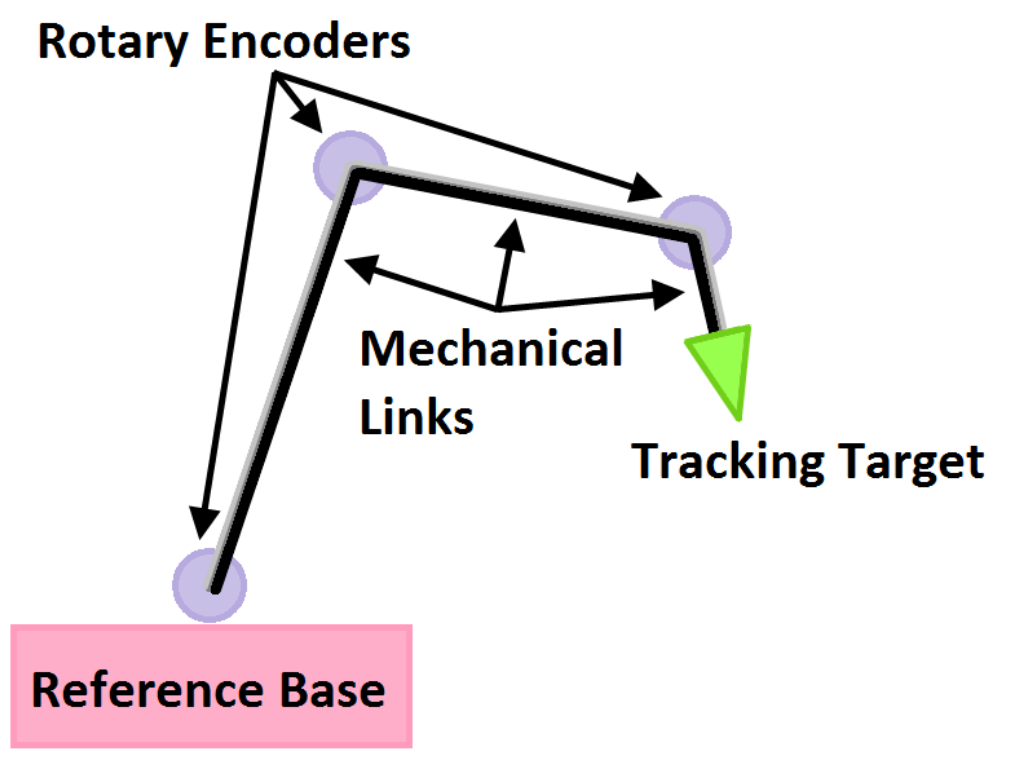

Figure 2.1: Diagram of a mechanical arm linking a reference location with target location.

Table 2.1 presents three popular mechanical tracking systems that have found use in the medical field over the past 20 years. Each new generation of system shows significant improvement in tracking performance, with the newest products achieving an accuracy and repeatability on the order of tens of microns at refresh rates in excess of 40,000 points per second [10]. When the need for ultra-high accuracy is paramount, no other available tracking method can approach this level of performance. 
Table 2.1: Mechanical Arm Tracking Systems.

\begin{tabular}{|l|l|l|l|}
\hline System & $\begin{array}{l}\text { Date } \\
\text { Released }\end{array}$ & Operating Sphere & Accuracy \\
\hline ISG Viewing Wand & 1994 & $640 \mathrm{~mm}$ radius & $\begin{array}{l}1.8 \mathrm{~mm}[6] \\
2.5-3.0 \mathrm{~mm}[7]\end{array}$ \\
\hline $\begin{array}{l}\text { FaroArm Bronze } \\
\text { Series Model 06 }\end{array}$ & 2004 & $1800 \mathrm{~mm}$ radius & $\begin{array}{l} \pm 0.15 \mathrm{~mm}[8] \\
\pm 0.43 \mathrm{~mm}(95 \%)[9] \\
\pm 0.66 \mathrm{~mm}(99 \%)[9]\end{array}$ \\
\hline FaroArm Fusion & 2009 & $1800 \mathrm{~mm}$ radius & $0.036 \mathrm{~mm} \mathrm{[10]}$ \\
\hline
\end{tabular}

For most medical device tracking applications, such as laparoscopic instrument tracking, the addition of a bulky mechanical arm and restrictions on the user's range of motion prove to be unacceptable constraints. There are many procedures for which a noncontact approach is required with the two most common options being electromagnetic and optical tracking.

\subsection{Electromagnetic (EM) Tracking Systems}

Electromagnetic tracking systems operate on the principle of sensing the strength of an electricallygenerated magnetic field. Field sensing is accomplished by sending current through three coils of wire contained in a small sensing probe where each coil is oriented orthogonally to the others. When current flows through a coil, it creates a second electromagnetic field that interacts with the stronger field created by the field generator. The strength of the resulting field at the coil location can then be recorded and through sequential activation of each coil and measurement of the magnetic field strength along each of three axes, position and orientation of the coil probe can be determined.

Among the more common EM medical tracking solutions are the LIBERTY Electromagnetic Motion Tracking System produced by Polhemus and the Aurora Electromagnetic Measurement System from Northern Digital Inc. (NDI). NDI recently acquired Ascension Technology Corporation, which produces the TrakSTAR Magnetic Tracker. Ascension also produced the microBIRD 6-DOF EM tracker that will be used as the reference system throughout this research. Though the microBIRD has been discontinued, the performance of this system remains comparable to current EM tracking solutions. Table 2.2 compares these three electromagnetic tracking systems. 


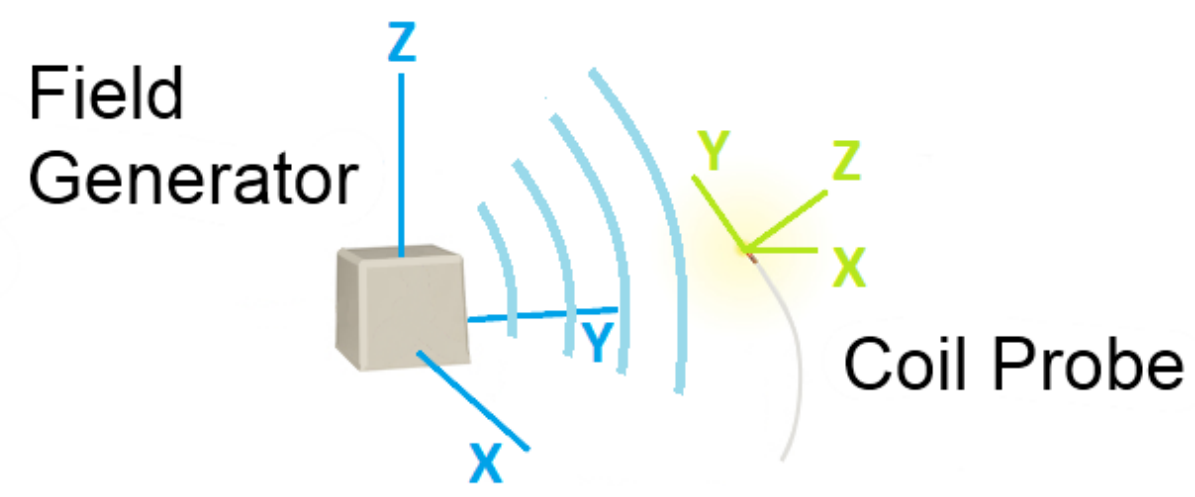

Figure 2.2: Diagram of an electromagnetic tracking system with field generator and sensor probe.

Table 2.2: Comparison of three electromagnetic tracking systems.

\begin{tabular}{|l|l|l|l|l|l|l|}
\hline \multirow{2}{*}{ System } & Radial Distance from & \multicolumn{2}{|l|}{ Position Accuracy } & Orientation Accuracy & \multirow{2}{*}{$\begin{array}{l}\text { Update } \\
\text { Rate }\end{array}$} \\
\cline { 3 - 6 } & Field Generator & RMS & $95 \%$ & RMS & $95 \%$ & $90 \mathrm{~Hz}$ \\
\hline $\begin{array}{l}\text { Ascension } \\
\text { microBIRD }\end{array}$ & $305 \mathrm{~mm}$ & $1.4 \mathrm{~mm}$ & - & $0.5^{\circ}$ & - & $120 \mathrm{~Hz}$ \\
\hline $\begin{array}{l}\text { Polhemus FAS- } \\
\text { TRAK [12] }\end{array}$ & - & $0.71 \mathrm{~mm}$ & - & $0.15^{\circ}$ & - & $40 \mathrm{~Hz}$ \\
\hline NDI Aurora [13] & $\begin{array}{l}250 \mathrm{~mm} \\
450 \mathrm{~mm}\end{array}$ & $\begin{array}{l}0.9 \mathrm{~mm} \\
1.6 \mathrm{~mm}\end{array}$ & $\begin{array}{l}2.0 \mathrm{~mm} \\
3.0 \mathrm{~mm}\end{array}$ & $\begin{array}{l}0.8^{\circ} \\
1.1^{\circ}\end{array}$ & $\begin{array}{l}1.5^{\circ} \\
1.7^{\circ}\end{array}$ & 40 \\
\hline
\end{tabular}

Capable of very high accuracy and low latency, EM technology has become the gold standard noncontact tracking solution, where accessibility or line-of-sight restrictions could cause competing technologies to fail.

One of the biggest drawbacks with electromagnetic tracking is that the operating environment must be free from magnetic and ferromagnetic material. Anything that generates or perturbs magnetic fields will degrade system performance. Additionally, electromagnetic trackers have a limited operating volume with accuracy and precision degrading as the radial distance from the field generator increases. Finally, EM tracking solutions are expensive, often costing tens of thousands of dollars. 


\subsection{Optical Tracking Systems}

Optical tracking is an alternate approach that addresses the limitations of EM tracking. An optical tracker operates by detecting unique features in images and relating the position of these features to the coordinate frame of the camera. These unique features, or fiducial markers, are affixed to the object to be tracked and can take the form of uniquely shaped markers, coloured or reflective patches (passive markers) or light-emitting tags such as LED markers (active markers). Fiducial markers that are easy to detect (have the greatest signal-to-noise ratio) will yield the most accurate and repeatable results.

To recover 3-D depth information, an optical tracking system typically uses two or more cameras mounted at known positions from each other. Images are simultaneously captured and the disparity between fiducial marker locations in each frame allows the 3-D position of each marker to be triangulated, as shown in Figure 2.3. By using two or more fiducial markers at different locations, a 3-D vector can be constructed and orientation information can be recovered. To obtain 6-DOF pose information using this technique, a minimum of three fiducial markers are typically used.

Some of the more common stereoscopic optical systems used for medical device tracking include the Polaris Spectra by NDI and the MicronTracker 3 by Claron Technology. Table 2.3 presents a comparison of the specifications for both systems.

From Table 2.3, it can be seen that these systems operate with an accuracy several times better than EM tracking solutions across a larger operating volume. Optical trackers can also operate near ferromagnetic material and in magnetically noisy environments, such as near magnetic resonance imaging (MRI) machines. The trade-off when capturing and analyzing camera images is that fast measurement rates are not easily achieved and lag is often noticeable.

The biggest disadvantage of optical tracking is object occlusion. If the line-of-sight between the camera and fiducial markers is lost, pose information cannot be computed. In a dynamic operating room environment, maintaining line-of-sight is not always possible and procedures must be designed with fiducial marker visibility in mind. This can restrict a surgeon's range of motion and complicate the surgical process. Since EM trackers do not require a line-of-sight, their use is often preferable to optical approaches. 


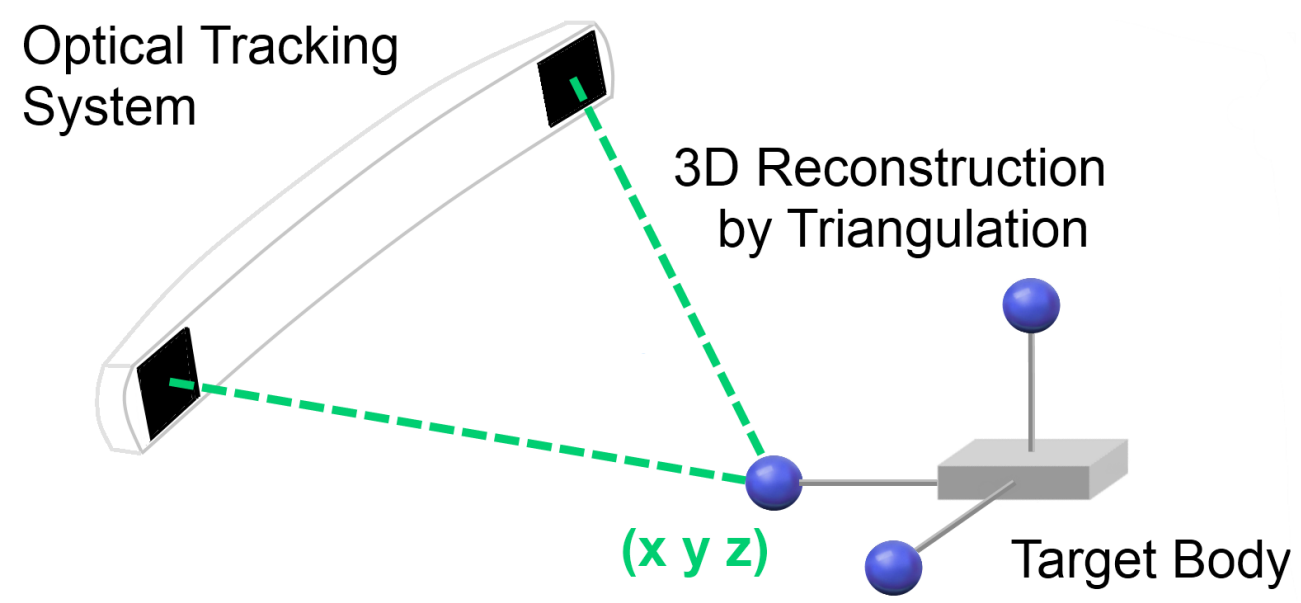

Figure 2.3: Triangulation method for an optical tracking system.

Table 2.3: Comparison of optical tracking systems with similar fields of measurement (FOM).

\begin{tabular}{|l|l|l|l|l|}
\hline System & $\begin{array}{l}\text { FOM, spherical section } \\
(\text { radius } \times \text { width } \times \text { height })\end{array}$ & $\begin{array}{l}\text { Position Accuracy } \\
(\text { RMS })\end{array}$ & $\begin{array}{l}\text { Update } \\
\text { Rate }\end{array}$ & Lag \\
\hline $\begin{array}{l}\text { Claron Technology [14] } \\
\text { MicronTracker 3 (H3-60) }\end{array}$ & $240 \times 200 \times 160 \mathrm{~cm}$ & $0.20 \mathrm{~mm}$ & $16 \mathrm{~Hz}$ & $60 \mathrm{~ms}$ \\
\hline $\begin{array}{l}\text { NDI Passive Polaris } \\
\text { Spectra [15] }\end{array}$ & $240 \times 157 \times 131 \mathrm{~cm}$ & $0.25 \mathrm{~mm}$ & $60 \mathrm{~Hz}$ & - \\
\hline
\end{tabular}

\subsubsection{SIMIS Vision Tracker}

While stereoscopic camera systems are the most common form of vision-based tracking, there are other methods that may be used to recover position and orientation from a camera system. A paper published by Tonet et al. in 2007 [16] presented one such alternative, whereby a single laparoscopic camera could be used to recover position as well as azimuth and elevation angles through image segmentation and shape-based image processing. This method exploited the perspective of a cylindrical instrument shaft captured in a camera frame allowing for the recovery of 5-DOF pose through segmented shape analysis.

Through continued work by Andrew Lyle at Canadian Surgical Technologies and Advanced Robotics (CSTAR), the work by Tonet et al. was augmented with roll angle estimation through the addition of two unique fiducial markers separated by 180 degrees about the cylindrical shaft of the instrument [17]. Image segmentation and processing of these markers mapped onto a cylindrical surface allowed for the recovery of instrument roll. Figure 2.4 shows an example image captured 
by the laparoscopic camera with image processing overlay to recover 6-DOF pose information.

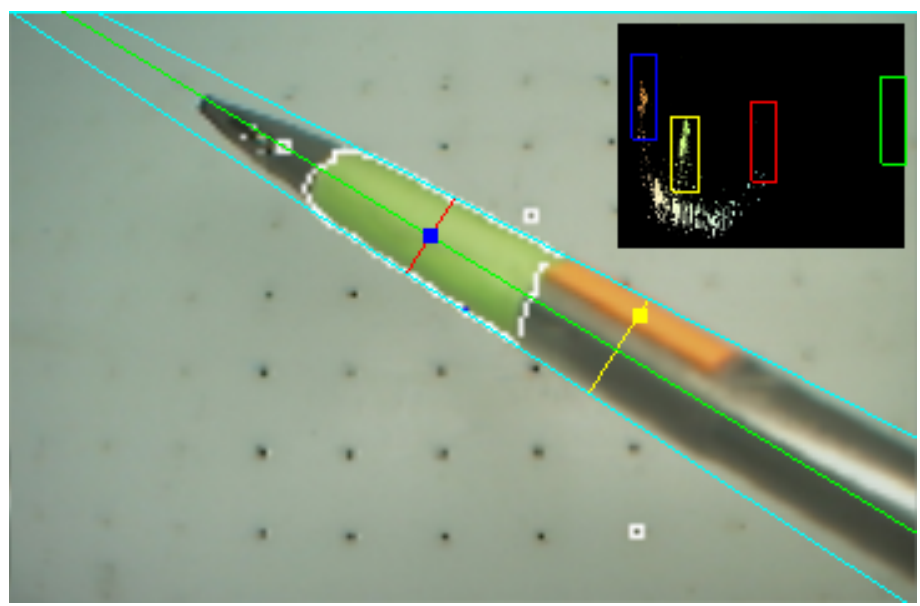

Figure 2.4: Image segmentation and analysis of a laparoscopic camera frame to recover 6-DOF pose information for the SIMIS vision tracker.

Independent testing of the SIMIS vision tracker involved a comparison of pose estimates against a mechanical jig, providing an absolute position and orientation reference. Results showed average positional RMS error of $7.4 \pm 0.6 \mathrm{~mm}$ with the newly-developed roll angle estimate displaying RMS error of $7.9 \pm 1.8^{\circ}$. Furthermore, the time required for image capture and analysis was not constant, with sampling rates fluctuating between 12-30 frames per second. Since the SIMIS vision tracker remains a project under development, it is expected that the performance of this system will improve. It should also be noted that, while the performance of this approach is inferior to other tracking systems, its main advantage is that it works using only a single camera - a camera that is often already present during MIS procedures.

\subsection{Alternate Approaches to Instrument Tracking}

Additional approaches exist to solve the problem of position and orientation estimation for medical devices. A wealth of published literature can be found that explores alternative approaches such as the use of ultrasound and fluoroscopics, yet the two most common approaches remain the use of optical and electromagnetic tracking.

Since any optical or image-based tracking system is susceptible to occlusion and EM trackers 
are susceptible to field distortions from magnetic and ferromagnetic material, neither approach represents a robust and reliable solution in all cases. In the following sections, alternate approaches that make use of MEMS-based inertial sensing are explored, as this technology has the potential to operate in harsh environments and provide significant cost savings over existing solutions.

\subsection{MEMS Technology}

Microelectromechanical systems (MEMS) is a technology involving the miniaturization of mechanical and electromechanical devices and structures. Before the advent of MEMS, inertial sensing could only be accomplished using high-precision mechanical gyros and accelerometers. These systems tended to be bulky and very expensive. MEMS devices, in contrast, typically range from 20 micrometres to 1 millimetre in size and often incorporate an on-board processing unit and other input/output components onto a single chip. Since MEMS devices can themselves be so small, the size of a commercial MEMS device package is often much larger than necessary to facilitate fabrication and the integration of these devices into other systems. MEMS micro-fabrication processes closely mirror those of semiconductor device fabrication, allowing rapid advances in the industry over the past few decades. This has caused a shift away from the older mechanical systems towards these new generations of small, inexpensive and often high performance sensors. Some of the more common types of MEMS devices used in sensing applications include pressure sensors, microphones, magnetometers and inertial sensors such as accelerometers and gyroscopes.

\subsection{Inertial Measurement Units (IMU)}

Used primarily as a navigation tool, an inertial measurement unit (IMU) is a broad term used to describe a package of electronic sensors that, at minimum, include a set of accelerometers and gyroscopes. These sensors are used to provide a 3-D measurement of inertial force (gravitational and/or specific force) and angular rate of rotation. An inertial navigation system (INS) is one type of IMU that uses accelerometers and gyroscopes to calculate velocity and position based on dead reckoning, while an attitude-heading reference system (AHRS) is a second class of IMU. Unlike an INS, an AHRS typically uses a collection of lower grade MEMS sensors and is augmented 
with a three-axis magnetometer to provide roll, pitch and yaw orientation information. An AHRS is, by definition, not capable of calculating position information. Both of these systems will be investigated in the following two sections.

\subsubsection{Inertial Navigation Systems (INS)}

At the outset of this research, the motivation and research goals were quite different. Initially, the problem of having a MIS instrument leave the field of view (FOV) of a laparoscopic camera was identified. It was noted that for basic laparoscopic motions such as suturing, the instrument was never out of the FOV of the camera for more than a few seconds, but when it was, the surgeon was effectively working blind with no visual reference or pose information. Thus, the initial motivation of this research was to develop a low-cost system that could be used to track the pose of the instrument for these few crucial seconds until the instrument could re-enter the FOV of the laparoscopic camera. By fusing an existing laparoscopic vision tracking system such as the SIMIS vision tracker with another technology, the aim was to create an inexpensive, continuous tracking solution.

An inertial navigation system is a navigation aid that uses accelerometers and gyroscopes to continually calculate the pose and velocity of a body without the need for an external reference. This process is known as dead reckoning. An initial pose and velocity must be provided to the INS, after which the orientation is tracked by integrating the angular rates of the gyroscopes at each time step. With an accurate estimate of INS orientation with respect to the Earth frame of reference, gravitational effects can be removed and inertial forces acting on the accelerometers can be integrated to track changes in velocity and position. Since an INS has no need for an external reference, it can be designed to be highly immune to environmental factors such as magnetic fields and operate under a wide range of conditions.

The biggest problem encountered when using inertial sensing systems for dead reckoning is drift. Small errors, primarily introduced by the accelerometers and gyroscopes at each time step, are integrated to become velocity and positional errors that cause accuracy degradation with each subsequent iteration. The longer an INS is left running, the more divergent the INS solution becomes from the true state. 
Drift is the result of all system and sensor errors within an INS. Beyond factors such as noise performance and quantization error of the analog-to-digital converter (ADC), inertial sensors are most commonly prone to bias (zero-value offset), scaling and misalignment error. In an accelerometer, these sources of error will misreport the inertial forces acting on the system, causing integration errors in the output velocity and position. Based on INS analysis methods developed by Tarrant et al. [18] and Collin et al. [19], sensor quality can be assessed by examining the positional drift due to specific sources of error. Table 2.4 presents the horizontal drift that can be expected for navigation-, tactical-, industrial- and automotive-grade accelerometers due to bias error. Similarly, Table 2.5 presents the effect of accelerometer misalignment as it relates to horizontal positional error. The equations used to generate this data will be discussed in more detail in Section 3.2.1.1.

Table 2.4: Accelerometer bias error expressed in $\mathrm{mg}\left(9.81 \times 10^{-3} \mathrm{~m} / \mathrm{s}^{2}\right)$.

\begin{tabular}{|l|l|l|l|l|l|}
\hline & \multirow{2}{*}{ Accelerometer } & \multicolumn{4}{|c|}{ Horizontal Positional Error } \\
\cline { 3 - 6 } Grade & Bias Error $(\mathrm{mg})$ & $1 \mathrm{~s}$ & $10 \mathrm{~s}$ & $60 \mathrm{~s}$ & $1 \mathrm{hr}$ \\
\hline Navigation & 0.025 & $0.13 \mathrm{~mm}$ & $12 \mathrm{~mm}$ & $0.44 \mathrm{~m}$ & $1.6 \mathrm{~km}$ \\
\hline Tactical & 0.3 & $1.5 \mathrm{~mm}$ & $150 \mathrm{~mm}$ & $5.3 \mathrm{~km}$ & $19 \mathrm{~km}$ \\
\hline Industrial & 3 & $15 \mathrm{~mm}$ & $1.5 \mathrm{~m}$ & $53 \mathrm{~m}$ & $190 \mathrm{~km}$ \\
\hline Automotive & 125 & $620 \mathrm{~mm}$ & $60 \mathrm{~m}$ & $2.2 \mathrm{~km}$ & $7900 \mathrm{~km}$ \\
\hline
\end{tabular}

Table 2.5: Accelerometer misalignment error.

\begin{tabular}{|l|l|l|l|l|l|}
\hline & Accelerometer & \multicolumn{4}{|c|}{ Horizontal Positional Error } \\
\cline { 3 - 6 } Grade & Misalignment $\left(^{\circ}\right)$ & $1 \mathrm{~s}$ & $10 \mathrm{~s}$ & $60 \mathrm{~s}$ & $1 \mathrm{hr}$ \\
\hline Navigation & 0.05 & $4.3 \mathrm{~mm}$ & $0.43 \mathrm{~m}$ & $15 \mathrm{~m}$ & $57 \mathrm{~km}$ \\
\hline Tactical & 0.1 & $8.6 \mathrm{~mm}$ & $0.86 \mathrm{~m}$ & $31 \mathrm{~m}$ & $110 \mathrm{~km}$ \\
\hline Industrial & 0.5 & $43 \mathrm{~mm}$ & $4.3 \mathrm{~m}$ & $150 \mathrm{~m}$ & $570 \mathrm{~km}$ \\
\hline Automotive & 1 & $86 \mathrm{~mm}$ & $8.6 \mathrm{~m}$ & $310 \mathrm{~m}$ & $1100 \mathrm{~km}$ \\
\hline
\end{tabular}

Gyroscopes measure rates of angular rotation in degrees per second or radians per second. In an INS, the sum of these integrated rates at each time step is used to update an orientation estimate of the sensing platform; this estimate can be used to rotate accelerometer measurements into the Earth frame for integration into velocity and position estimates across Earth's surface. While gyro error will directly affect the orientation estimate and subsequently the position estimate of the INS, 
Table 2.6: Gyro angle random walk error.

\begin{tabular}{|l|l|l|l|l|l|}
\hline & Gyro Angle Random & \multicolumn{4}{|c|}{ Horizontal Positional Error } \\
\cline { 3 - 6 } Grade & Walk (ARW) $(\circ / \sqrt{h r})$ & $1 \mathrm{~s}$ & $10 \mathrm{~s}$ & $60 \mathrm{~s}$ & $1 \mathrm{hr}$ \\
\hline Navigation & 0.002 & $0.01 \mathrm{~mm}$ & $0.1 \mathrm{~mm}$ & $1.3 \mathrm{~mm}$ & $620 \mathrm{~m}$ \\
\hline Tactical & 0.07 & $0.1 \mathrm{~mm}$ & $3.2 \mathrm{~mm}$ & $46 \mathrm{~mm}$ & $22 \mathrm{~km}$ \\
\hline Industrial & 3 & $10 \mathrm{~mm}$ & $0.23 \mathrm{~m}$ & $3.3 \mathrm{~m}$ & $1500 \mathrm{~km}$ \\
\hline Automotive & 5 & $20 \mathrm{~mm}$ & $0.45 \mathrm{~m}$ & $6.6 \mathrm{~m}$ & $3100 \mathrm{~km}$ \\
\hline
\end{tabular}

integration of gyro signals containing white noise with a Gaussian distribution also has a special effect on angle estimates, known as gyro angle random walk (ARW). By multiplying the ARW with the root of the integration time step, the 1-sigma walk angle can be determined for any gyro and is often the parameter used to assess gyro quality. Based on the work by Nebot et al. [20], INS drift due to gyro ARW was calculated and presented in Table 2.6. If navigation-grade accelerometers and gyroscopes are used, ARW can be one of the leading causes of INS drift; however, for lower quality sensors, accelerometer bias error quickly dominates.

The data in this section provides a foundation for the INS sensor selection case study presented in Chapter 3.

\subsubsection{Attitude-Heading Reference Systems (AHRS)}

Since an attitude-heading reference system tends to use lower quality commercial- or automotivegrade sensors, its operation as a navigation aid is fundamentally different than that of an INS and is limited to providing orientation information. An AHRS operates by using a three-axis accelerometer to sense the direction of Earth's gravity vector and a three-axis magnetometer to sense the direction of Earth's magnetic field. With these two reference vectors, roll, pitch and yaw angles of the sensing platform with respect to the Earth frame can be computed. A sensor fusion algorithm is required to generate these orientation estimates, often requiring that the system include a chip capable of on-board digital signal processing.

Accelerometers sense all accelerations including vibration and noise. With a body in motion, the accelerometer is often unable to provide a clean estimate of the gravitational vector due to the presence of other inertial forces. Since magnetometer data must be rotated into a frame parallel 
to the Earth's surface before the compass heading (yaw angle) can be computed, accelerometer data must be used to determine this rotation. This means that heading estimates are susceptible to sources of error from both the accelerometer and magnetometer.

While gyroscopes are not technically required to compute orientation, the addition of angular rate sensing has a profound effect on the performance and reliability of an AHRS. Knowing that the integration of angular rates provides a second means of determining orientation, these estimates can be used to smooth out estimates provided by the other sensors. By using gyros to track the orientation of the sensing platform with respect to the downward gravitational vector, corrected magnetometer readings can provide a much cleaner estimate of the yaw angle, free from accelerometer influence. Furthermore, since gyros are prone to random walk and will drift over time, small accelerometer and magnetometer influence can be used to correct any accumulated angular error, thereby guaranteeing that orientation estimates remain drift free. A well-designed sensor fusion algorithm can provide a robust and efficient orientation estimate.

Work by Ren et al. [21] [22] investigated the use of an AHRS to track the orientation of laparoscopic instruments and found that inexpensive MEMS devices could achieve orientation accuracy error below $1^{\circ}$ [23]. This work also investigated the fusion of an AHRS with an EM tracker to provide 6-DOF pose information in much the same way that global positioning system (GPS) data can be fused with an INS to improve the performance of the navigation system [24] [25].

The work presented in this thesis differs from the work by Ren et al. in a number of important ways. The algorithm developed in Chapter 3 applies the concept of an AHRS augmented with distance sensing to track the orientation and position of a laparoscopic instrument without the need for a second reference tracking system. As a result, the modified laparoscopic instrument and augmented sensor fusion algorithm can be considered to be the most significant contributions of this work.

\subsection{Summary}

In this chapter, leading medical tracking technologies were presented; specifically, mechanical, optical and electromagnetic tracking systems were investigated. The advantages and limitations 
of each approach were discussed and the need for an alternative tracking method was identified. Inertial navigation systems and attitude-heading reference systems were also presented as these technologies introduce the concept of inertial sensing and lay the foundation for the work presented in Chapter 3. 


\section{Chapter 3}

\section{System Design}

\subsection{Coordinate Frames and Conventions}

To begin this discussion, the problem of inertial sensing will be simplified by assuming a flat Earth model. Since this research is confined to orientation and position sensing across less than a few metres, this assumption is acceptable. As shown in Figure 3.1, the first coordinate frame will be a local level coordinate frame where one axis is parallel to the Earth's surface in the direction of magnetic north, with a second perpendicular axis pointing to the east. Following the right-handed convention, the third axis of this system points downward to complete the north-east-down (NED) coordinate system ( $N$ frame).

From the $N$ frame, the local level, wander azimuth frame ( $L$-frame) can be defined with $x$ and $y$ axes parallel to the Earth's surface and the $z$ axis pointing downward to complete a right-handed frame. This coordinate frame is free to rotate about the $z$ axis and the $x$ and $y$ axes may not be aligned with the north and east directions as in the $N$ frame. In this way, the azimuth angle is said to wander and the frame's orientation is nonsingular. A strap-down magnetometer sensing the Earth's magnetic field must be rotated into the $L$ frame before it can be used for compassing to sense heading angle.

The body-fixed frame ( $B$ frame) is one that is fixed to the inertial sensing platform. The $B$ frame is free to rotate in 3 -space as the sensing platform rotates with respect to the $N$ and $L$ frames. 


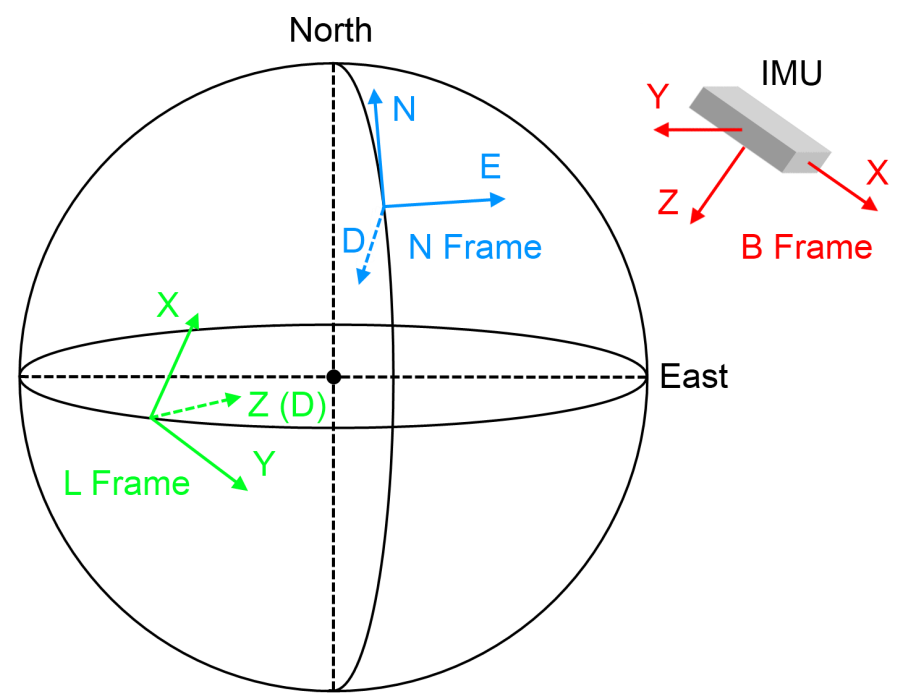

Figure 3.1: N, L and B coordinate frame conventions.

Finally, the way the sensors are mounted to the platform leads to small misalignment errors between the axes of each sensor $\left(S_{n}\right.$ frame) and the $B$ frame. Proper calibration of the inertial sensing package will correct for these misalignment errors so that the $S_{n}$ frames are aligned with the $B$ frame. In the absence of misalignment error, the sensors can be considered to be in the $B$ frame.

\subsection{Hardware System Design}

In this section, an inertial measurement unit containing accelerometers, magnetometers and gyroscopes will be selected and augmented with a distance sensor to complete a hardware platform from which 6-DOF pose information of a laparoscopic instrument can be derived. Final pose estimate quality is highly dependent on sensor performance and this section discusses the sensor selection process with quantitative analyses. A case study is presented for INS sensor selection which may become useful for future work involving inertial sensing. Much of this discussion will remain equally applicable to an AHRS. Finally, sensor selection for an AHRS is conducted and the hardware implementation is presented. 


\subsubsection{INS Sensors}

Theoretically, the concept of an INS represents the ideal tracking solution. This self-contained system does not require external input to determine position and orientation making it immune to environmental interference. Applied to medical device tracking, this idealized INS would not suffer occlusion and could operate in magnetically noisy environments.

Acknowledging that real-world INS experience drift, this research project was initially focused on examining the viability of using an INS coupled with another tracking technology to provide 6-DOF pose information. By minimizing reliance on external input, the goal was to drastically improve interference rejection while guaranteeing a drift-free solution. Results of this investigation concluded that current-generation INS are not yet practical for unaided laparoscopic instrument tracking.

Through the design and selection of sensors for an INS, major sources of accelerometer and gyroscope error can be investigated. These concepts will remain largely applicable to the final AHRS implementation presented in this thesis and, as sensor performance continues to increase, it may soon become possible to implement an INS for medical device tracking.

\subsubsection{Sensor Selection Case Study}

This case study will explore the integration of an INS with a vision-based tracking system. The SIMIS vision tracker presented in Chapter 2 is an experimental 6-DOF laparoscopic instrument tracking system that uses a single laparoscopic camera. The narrow field of view of this camera means the laparoscopic instrument often leaves the camera frame when performing routine procedures. To solve this problem, an INS will be used to track the instrument during these "blackout" periods where no pose information is available from SIMIS. These periods may last 2-3 seconds during which time the INS must not exceed a positional error of $1 \mathrm{~cm}$. Through numerical analysis and comparison to existing INS, sensor specifications will be determined that limit drift performance to $\leq 1 \mathrm{~cm}$ in 3 seconds.

Since drift performance (positional error) of an INS is the result of any and all errors introduced by the sensors, a useful INS requires very high-performance components. To characterize this 
performance, mathematical relationships must be defined that relate the main sources of sensor error to the resulting drift.

Accelerometer Bias The single largest source of positional drift for a MEMS-based INS is due to accelerometer offset errors. Positional error, $d_{\mathrm{error}}$, can be modeled as a function of accelerometer bias, $a_{\mathrm{b}}$, by the following kinematic equation:

$$
d_{\text {error }}=\frac{1}{2} \cdot g \cdot a_{\mathrm{b}} \cdot \Delta t^{2}
$$

where, $g$ is the acceleration due to gravity and $t$ is time in seconds. Substituting $d_{\text {error }}=0.01 \mathrm{~m}$, $t=3 \mathrm{~s}$ and $g=9.81 \frac{\mathrm{m}}{\mathrm{s}^{2}}$, we can rearrange to find that $a_{\mathrm{b}}=2.3 \times 10^{-4} \mathrm{~g}$. If the magnitude of cumulative offset error from each axis exceeds $230 \mu \mathrm{g}$, the integrated positional drift will become unmanageable.

Accelerometer Axis Misalignment Another source of accelerometer error is due to axis misalignment. Even if there were no other source of orientation error and the sensing platform ( $B$ frame) was perfectly aligned with the $N$ frame, accelerometer axis misalignment, $a_{\mathrm{m}}$, in the $S$ frame would misrepresent the true inertial forces experienced along each body axis. This misalignment can cause sensing of off-axis inertial forces and lead to positional error modeled by the following equation:

$$
d_{\text {error }}=\frac{1}{2} \cdot g \cdot \sin \left(a_{\mathrm{m}}\right) \cdot \Delta t^{2}
$$

By substituting the same parameters as in Equation 3.1, it can be determined that $a_{\mathrm{m}}=0.013^{\circ}$. If the magnitude of cumulative offset error from each axis exceeds $0.013^{\circ}$, it can be expected that the resulting drift will not satisfy the design criteria.

Gyro Random Walk The other leading source of INS drift is due to gyro angle random walk, $\dot{\theta}_{\mathrm{RW}}$. Often represented in terms of $\% / \sqrt{\mathrm{hr}}$ or $\% / \mathrm{min}, \dot{\theta}_{\mathrm{RW}}$ is the rate of accumulated orientation error caused by integrating gyro angular rate signals containing white noise. The relationship between positional error and gyro random walk can be described by the following equation [20]: 


$$
d_{\text {error }}=\frac{1}{6} \cdot g \cdot \sin \left(\dot{\theta}_{\mathrm{RW}}\right) \cdot \Delta t^{3} \text {. }
$$

Substituting the same parameters as those used with Equation 3.1 yields a maximum gyro angular walk of $0.013 \%$ s or $47^{\circ} / \mathrm{hr}$.

Some additional sources of sensor error include temperature drift, gyro offset and misalignment, scaling errors and nonlinear signal response. The three main sources of error presented in detail here represent the most significant contributors to INS drift. It must be noted, however, that these error sources are not independent from one another. The following parameters present a second scenario in which each sensor error contributes equal thirds to $1 \mathrm{~cm}$ total drift in 3 seconds:

$$
\begin{array}{lll}
a_{\mathrm{b}} & =44 \mu \mathrm{g} & \text { along each of } 3 \text { axes } \\
a_{\mathrm{m}} & =0.0025^{\circ} & \text { along each of } 3 \text { axes } \\
\dot{\theta}_{\mathrm{RW}} & =15.6^{\circ} / \mathrm{hr} &
\end{array}
$$

In this case, error is evenly distributed between each axis of the 3-axis sensors and the resulting drift directions are collinear — an unlikely scenario though not impossible. Since a real system presents additional sources of error, these values are only meant to quantify a range around which the target INS sensors must perform.

A proper INS calibration routine is able to estimate and correct for factors such as axis misalignment, scaling factors and, at the time of initialization, sensor offsets, which can greatly improve the performance of the system. Accelerometers, for example, are particularly susceptible to temperature drift that can affect the zero-g output signal; however, by including temperature monitoring, a relationship for this thermally-coupled offset can be estimated. Even after careful calibration and modeling of sensor error, a perfect estimate is never possible and performance can only be improved through the use of higher quality sensors at a higher cost.

Considering some of the target sensor parameters that have been quantified, a survey of existing systems reveals that these specifications are not easily satisfied. Table 3.1 presents a sample of MEMS-based INS ranging from commercial through to tactical-grade. The cheapest of these systems costs several thousand dollars. Only the most expensive, tactical-grade INS, the STIM300, provides acceptable sensor performance. While the STIM300 is one of the smallest INS in its 
Table 3.1: Examples of commercially-available MEMS-based INS solutions.

\begin{tabular}{|c|c|c|c|}
\hline & $\begin{array}{ll}\text { VectorNav } & \text { VN-200 } \\
\text { INS/GPS [26] } & \\
\end{array}$ & $\begin{array}{ll}\text { SBG Systems IG- } \\
500 \mathrm{~N}[27]\end{array}$ & $\begin{array}{ll}\text { Sensonor } & \text { STIM300 } \\
{[28]} & \\
\end{array}$ \\
\hline \multicolumn{4}{|l|}{ Accelerometer } \\
\hline $\begin{array}{l}\text { Range } \\
\text { Resolution } \\
\text { Bias Instability } \\
\text { Noise Density } \\
\text { Bandwidth } \\
\text { Alignment Error }\end{array}$ & $\begin{array}{l} \pm 8 \mathrm{~g} \\
0.4 \mathrm{mg} / \sqrt{\mathrm{Hz}} \\
260 \mathrm{~Hz} \\
\pm 0.05^{\circ}\end{array}$ & $\begin{array}{l} \pm 5 \mathrm{~g} \\
\pm 0.06 \mathrm{mg} \\
0.25 \mathrm{mg} / \sqrt{\mathrm{Hz}} \\
250 \mathrm{~Hz} \\
<0.05^{\circ}\end{array}$ & $\begin{array}{l} \pm 10 \mathrm{~g} \\
1.9 \mu \mathrm{g} \\
0.05 \mathrm{mg}\end{array}$ \\
\hline \multicolumn{4}{|l|}{ Gyroscope } \\
\hline $\begin{array}{l}\text { Range } \\
\text { Resolution } \\
\text { Random Walk } \\
\text { Noise Density } \\
\text { Bandwidth } \\
\text { Alignment Error }\end{array}$ & $\begin{array}{l} \pm 500^{\circ} / \mathrm{s} \\
288^{\circ} / \mathrm{hr} \\
0.005(\% / \mathrm{s}) / \sqrt{\mathrm{Hz}} \\
256 \mathrm{~Hz} \\
\pm 0.05^{\circ}\end{array}$ & $\begin{array}{l} \pm 300^{\circ} / \mathrm{s} \\
20 \% / \mathrm{hr} \\
0.05(\% / \mathrm{s}) / \sqrt{\mathrm{Hz}} \\
240 \mathrm{~Hz} \\
<0.05^{\circ}\end{array}$ & $\begin{array}{l} \pm 400^{\circ} / \mathrm{s} \\
0.22^{\circ} / \mathrm{hr} \\
9^{\circ} / \mathrm{hr}\end{array}$ \\
\hline \multicolumn{4}{|l|}{ System } \\
\hline $\begin{array}{l}\text { Output Rate } \\
\text { Dimensions } \\
\text { Weight } \\
\text { Price }\end{array}$ & $\begin{array}{l}200 \mathrm{~Hz} \\
22 \times 24 \times 3 \mathrm{~mm} \\
3 \text { grams }(45 \text { grams } \\
\text { mounted on board }) \\
\$ \$\end{array}$ & $\begin{array}{l}100 \mathrm{~Hz}(10,000 \mathrm{~Hz} \\
\text { internal sampling }) \\
27 \times 30 \times 14 \mathrm{~mm} \\
10 \text { grams } \\
\$ \$ \$\end{array}$ & $\begin{array}{l}2000 \mathrm{~Hz} \\
44.8 \times 38.6 \times 21.5 \mathrm{~mm} \\
55 \text { grams } \\
\$ \$ \$ \$\end{array}$ \\
\hline
\end{tabular}

class, it is still too large and too heavy to mount on a laparoscopic instrument, making INS-based solutions impractical for the task of unaided instrument tracking.

This case study examined the use of a low-cost, instrument-mounted, INS package to augment the function of a vision-based tracking system and provide reliable pose information for up to 3 seconds. This analysis has shown that nothing short of a tactical-grade system will meet these objectives and at a price which rivals commercially-available medical tracking systems, the use of INS can not be justified. An alternate approach must be explored. 


\subsubsection{AHRS Sensors}

Inertial sensing packages can also be found in the form of an Attitude-Heading Reference System (AHRS). These systems provide orientation sensing of the body with respect to Earth's $N$ frame and are not capable of tracking position. The AHRS and INS both contain accelerometers and gyroscopes; however, unlike an INS, AHRS are almost always augmented with magnetometers for compassing based on Earth's magnetic field. An AHRS uses triads of each sensor to measure the downward gravity vector and direction of the Earth's magnetic field to generate an orientation estimate. Since these vectors are stable, a properly designed AHRS will not experience unbounded drift even over long periods of time. Furthermore, the AHRS need not be as precisely calibrated as an INS, meaning that much cheaper and lower-grade sensors may be used, while still maintaining an accurate and precise orientation estimate.

To accomplish AHRS orientation sensing, sensor data must be captured and processed (usually with an on-board microcontroller). The digital signal processing (DSP) algorithms of an AHRS largely determine the quality of the output estimate. By applying proper signal filtering methods, even industrial-grade sensors can be used to estimate 3-DOF orientation with an accuracy better than $1^{\circ}[23]$. Furthermore, this performance can be maintained even if the accelerometer and magnetometer signals are very noisy.

Accelerometers While the use of high-quality sensors is not necessary to achieve good AHRS performance, several sensor parameters are important and must be considered. Since a 3-axis accelerometer is used to provide an estimate of roll and pitch, sensor offsets and misalignment will still affect the accuracy of these estimates. Table 3.2 shows the effect of static accelerometer bias on roll and pitch errors for different sensor grades. Similarly, Table 3.3 shows the effect of accelerometer misalignment on roll and pitch errors.

Tables 3.2 and 3.3 present a worst-case scenario for sensors that have not been calibrated. A simple calibration routine for an automotive-grade accelerometer can reduce the pitch and roll errors by as much as two orders of magnitude, resulting in attitude errors below $0.1^{\circ}$. While accelerometer misalignment is easily corrected and can be considered constant for the life of the mounted sensor, accelerometer bias may experience deviation due to temperature changes, long- 
Table 3.2: Static error due to accelerometer bias.

\begin{tabular}{|l|l|l|}
\hline Grade & $\begin{array}{l}\text { Accelerometer Bias Error } \\
(\mathrm{mg})\left(9.81 \times 10^{-3} \mathrm{~m} / \mathrm{s}^{2}\right)\end{array}$ & $\begin{array}{l}\text { Pitch/Roll Error } \\
\left(^{\circ}\right)\end{array}$ \\
\hline Navigation & 0.025 & 0.0014 \\
Tactical & 0.3 & 0.017 \\
Industrial & 3 & 0.17 \\
Automotive & 125 & 7.2 \\
\hline
\end{tabular}

Table 3.3: Static error due to accelerometer misalignment.

\begin{tabular}{|l|l|}
\hline Grade & $\begin{array}{l}\text { Accelerometer Axis Alignment } \\
\left({ }^{\circ}\right)\end{array}$ \\
\hline Navigation & 0.0029 \\
Tactical & 0.0057 \\
Industrial & 0.057 \\
Automotive & 0.57 \\
\hline
\end{tabular}

term drift or other factors not considered in the sensor model. Of these factors, temperature effects for automotive and industrial-grade accelerometers represent the largest source of post-calibrated bias deviation and should be compensated for if highly accurate roll/pitch estimates are required.

Magnetometers Similarly, the quality and calibration of a 3-axis magnetometer directly affects heading accuracy. Magnetometers typically measure the strength of magnetic fields in units of gauss $(\mathrm{G})$ and at its surface, the Earth's magnetic field is $0.31-0.58$ gauss depending on location. If heading accuracy within $1^{\circ}$ is desired, the magnetometer output along a single axis must not deviate by more than $1.7 \%$ of its true value. For a local field strength of 0.54 gauss, this constrains axis error to no more than 9 milligauss. Magnetometers, like other sensors, are susceptible to offsets, scaling error (nonlinearity), misalignment as well as temperature effects. They also tend to exhibit higher levels of output noise than a similar grade accelerometer. It is not uncommon for an industrial grade magnetometer to perform compassing with an accuracy no better than $2-3^{\circ}$ RMS.

To explore magnetometer error, let us consider the Honeywell HMC6052 integrated compass sensor. From an analysis of this sensor's data sheet [29], it can be seen why heading errors $>1^{\circ}$ RMS are not uncommon for this grade of sensor. With a field sensitivity of $0.5 \mathrm{~V} /$ gauss, a noise 
floor of $1 \mathrm{mV}$ is equivalent to 2 milligauss. A linearity error of $0.4 \%$ across the full scale of this sensor ( -2.0 gauss to 2.0 gauss) is equivalent to 16 milligauss across the full scale. If the Earth's magnetic field is measured to be between -0.54 gauss and 0.54 gauss, this linearity error drops to 4.3 milligauss. A temperature sensitivity of $-2700 \mathrm{PPM} /{ }^{\circ} \mathrm{C}$ across a change of only $2^{\circ} \mathrm{C}$ can change the field sensitivity to $0.497 \mathrm{~V} /$ gauss, resulting in an error of 3 milligauss. Summing the errors of just these three sources, the possible error along a single axis is 9.3 milligauss. Considering both axes of this magnetometer, this results in a possible heading error of up to $1.4^{\circ}$. Finally, it is important to realize that magnetometers are sensitive to all magnetic fields and all perturbations of these fields. Current-carrying conductors will create their own interfering magnetic fields and any nearby ferromagnetic materials will distort the direction of these fields. In any practical application, it is important to realize that field distortions and magnetic noise will severely limit the accuracy of a magnetometer, regardless of the quality or price of the device.

Gyroscopes Since accelerometers and magnetometers can provide an attitude and heading reference, gyroscopes are not required for static orientation estimation; however, for real-world applications involving systems in motion, gyroscopes serve a very important function.

Accelerometers measure all accelerations, such that a body experiencing changes in velocity and subject to vibration will not be able to accurately calculate roll and pitch angles. The 6-12 $\mathrm{Hz}$ hand tremor induced on a hand-held accelerometer can perturb roll and pitch estimates by as much as $10^{\circ}$. Furthermore, although magnetometers are not susceptible to inertial force or vibration, their derived heading estimates are susceptible. A magnetometer mounted in the body frame will capture a magnetic field vector that must be rotated into the $L$ frame before it can be used for compassing. This can only be accomplished by using the accelerometer's roll and pitch estimates. As a result, heading accuracy is susceptible to inertial disturbances, sources of error and interference from both the accelerometers and magnetometers. This susceptibility can be dampened through the use of gyroscopes.

Angular rates from the gyroscopes can be integrated to provide a second, short-term orientation estimate for the AHRS based on previous estimates. Since gyroscopes exhibit random walk, this orientation estimate will degrade; however, over short periods of time, these orientation estimates 
tend to be very accurate. By fusing gyro orientation estimates with the noisier accelerometer and magnetometer estimates, an AHRS can exhibit drift-free performance as well as remain highly immune to environmental factors, vibration and noise. The simplest way to fuse these orientation estimates is known as a complementary filter; other filtering methods, such as the Kalman filter, offer better and more robust performance.

Since the accuracy of an AHRS is largely dependent on the sensor fusion algorithm being used, final system performance cannot be easily predicted based purely on theoretical analysis. Even properly calibrated automotive-grade sensors can achieve performance better than a fraction of a degree, which is acceptable for 3-DOF orientation tracking of a laparoscopic instrument. At this point, the factors driving sensor selection largely become size, weight and cost. The best way to minimize these factors is to mount the sensor on the instrument and move the data processing off-chip, saving the weight and power consumption of an on-board DSP.

Most AHRS are designed for vehicle motions and autonomous navigation and, as such, there are few applications in medical instrument tracking. As a result, an off-the-shelf AHRS may not provide the granularity of control and tunability required for a medical environment. To address this shortcoming, a customized AHRS algorithm must be developed.

\subsubsection{Phidgets 9-DOF IMU}

During the sensor selection and hardware design phases of this research, the sensing platform went through a number of iterations. In the first iteration, an Analog Devices ADXL345 3-axis digital accelerometer, a Honeywell HMC6043 3-axis magnetometer and two Inversense IDG-300 gyroscopes were mounted on a platform controlled by a microcontroller. A software layer was written to efficiently poll all of the sensors and an embedded AHRS algorithm was developed. Even after following proper system design practices and thorough calibration, the system did not exhibit satisfactory performance. The sampling rates were too slow and the IDG-300 gyros exhibited levels of drift higher than specified in the data sheet. The resulting gyro orientation estimates were less accurate than required and this system was abandoned in favour of better components.

After a thorough investigation of commercially available sensors, the STMicroelectronics LIS344 
accelerometer and the STMicroelectronics LPR510 gyroscope had been identified as the bestperforming devices in this class. At the same time, it was discovered that a Canadian-based company, Phidgets Inc., had just released the Spatial 1056 9-DOF IMU containing these sensors at a retail price of $\$ 150 \mathrm{CAD}$. This product included a 16 -bit $\mathrm{ADC}$, an external reference voltage chip and an on-board Atmel microcontroller capable of control and transmission over USB 2.0 at up to 250 samples per second. Finally, this device included a built-in magnetometer self recalibration routine to ensure that heading estimates would remain as accurate as possible, even over extended periods of use. The Spatial 1056 was purchased and used for the duration of this thesis with great success. Table 3.4 provides an overview of the specifications of the Phidgets Spatial 9-DOF IMU.

Table 3.4: Phidgets Spatial 1056 9-DOF IMU Specifications.

\begin{tabular}{|l|l|}
\hline Board & Phidgets Spatial 1056 9-DOF Sensor Module \\
\hline Current Consumption & $45 \mathrm{~mA}$ \\
Max Sample Speed & $4 \mathrm{~ms} / \mathrm{sample}$ \\
ADC Resolution & 16 -bit \\
Interface & USB 2.0 \\
Dimensions & $1.2^{\prime \prime} \times 1.4^{\prime \prime} \times 0.218^{\prime \prime}(30.5 \mathrm{~mm} \times 35.6 \mathrm{~mm} \times 5.54 \mathrm{~mm})$ \\
\hline Accelerometer & STMicroelectronics LIS344ALH \\
\hline Measurement Resolution & $228 \mu \mathrm{g}$ \\
Bandwidth & $110 \mathrm{~Hz}$ \\
Measurement Range & $\pm 5 \mathrm{~g}$ \\
Noise Performance & $300 \mu \mathrm{g}(x$ axis $), 300 \mu \mathrm{g}(y$ axis $), 500 \mu \mathrm{g}(z$ axis $)$ \\
Error Through & $2 \mathrm{mg}$ \\
Range $\left(360^{\circ}\right)$ & $\mathbf{S T M i c r o e l e c t r o n i c s ~ L P R 5 1 0 A L ~ ( 2 - A x i s ) ~}+$ \\
\hline Gyroscope & Y510AH (1-Axis) \\
\hline Full Scale Range & $\pm 400^{\circ} / \mathrm{s}$ \\
Resolution & $0.02^{\circ} / \mathrm{s}$ \\
Drift & $4{ }^{\circ} / \mathrm{min}$ \\
\hline Magnetometer $($ Compass $)$ & Honeywell HMC6042 (2-Axis) + (1-Axis) sen- \\
\hline sor \\
\hline Resolution & $400 \mu \mathrm{G}$ \\
Offset from North & $2^{\circ}$ \\
\hline
\end{tabular}




\subsubsection{Hardware Configuration}

Proper sensor selection and a well-designed AHRS algorithm can be used to generate accurate orientation estimates but a complete instrument tracking system must be able to resolve tip location as well as orientation.

The environment within which a laparoscopic instrument operates was examined and Figure 3.2 illustrates typical movement of a laparoscopic instrument across the tissue interface. Due to the nature of port-based procedures, tangential motion in the tissue plane is largely constrained leaving the instrument free to rotate about its long axis. Any translation of the port in the tissue plane will cause tissue deformation as the result of forces applied by the surgeon. Since tissue is an elastic material, deformation will cause the tissue to exert a reactionary force tending to return the instrument to a location of minimal deformation. This point represents a local energy minimum and it is reasonable to assume that for a typical laparoscopic procedure, this point will act primarily as a pivot as shown in Figure 3.2.

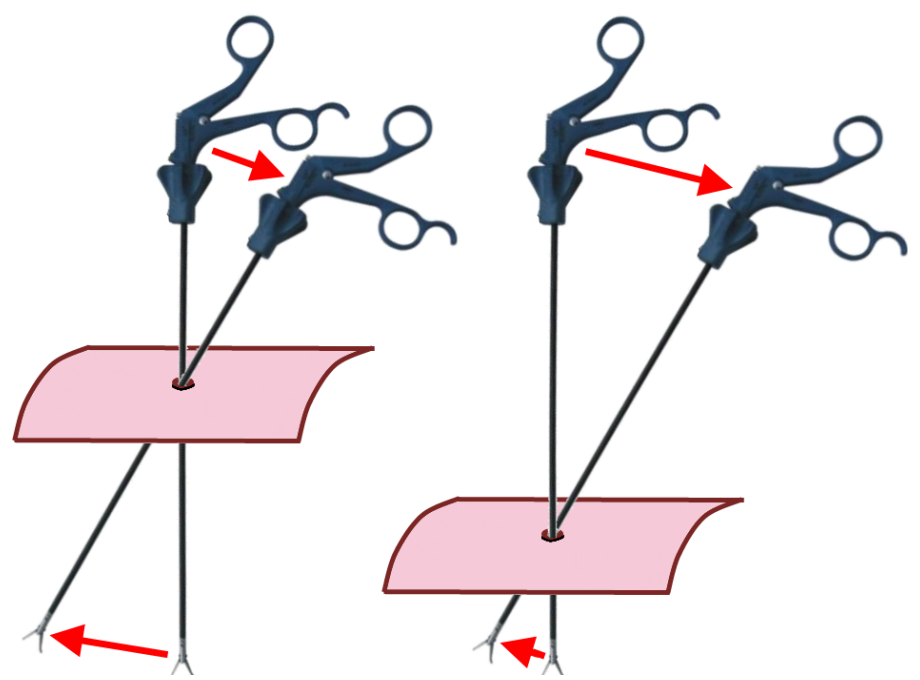

Figure 3.2: Movement of a laparoscopic instrument across the tissue layer.

Making the assumption that a point exists through which the position of the trocar is constrained, this point can be used as a datum from which further position information can be derived. Furthermore, in a surgical training environment, conditions can be controlled to ensure the validity of this assumption. As illustrated in Figure 3.2, for an instrument of fixed length, the ratio of 
length on each side of the pivot controls the scale of motion of the instrument. Since the orientation of a rigid body is constant at every point, by tracking the length of the instrument from pivot to tip, this length can be projected into 3-D Cartesian coordinates using the pivot as a datum.

A distance sensor can be used to determine this pivot-to-tip distance. To minimize interference with the normal operation of the instrument and ensure the full range of motion, the distance sensor must be mounted external to the patient.

\subsubsection{Sharp IR Distance Sensor}

Since all laparoscopic instruments must pass through a trocar, the distance from the trocar to instrument handle can be used to track the depth of an instrument with fixed length. Infrared (IR) distance sensors are low-cost devices capable of providing accurate, noncontact range measurements within a typical distance of 4 to 200 or more $\mathrm{cm}$. The Sharp GP2D120 IR sensor [30] is an analog device with a range of 4-30 cm and a response time of $39 \mathrm{~ms}$, allowing for a refresh rate of 26 samples per second. Given its size, weight and negligible cost, it is well suited for real-time tracking of instrument depth.

The Sharp GP2D120 IR sensor has a nonlinear distance to voltage relationship, as shown in Figure 3.3. Using the sensor's data sheet [30] and a $5 \mathrm{~V}$ power supply, this sensor can be expected to output $2.7 \mathrm{~V}$ at $4 \mathrm{~cm}$ and as little as $0.4 \mathrm{~V}$ at $30 \mathrm{~cm}$. An Atmel Mega328 with 10-bit, $5 \mathrm{~V}$ analog to digital converter (ADC) is capable of an average resolution of $0.05 \mathrm{~cm}$ per LSB with a close range resolution as high as $0.01 \mathrm{~cm}$ per LSB and far range resolution as low as $0.2 \mathrm{~cm}$ per LSB. Due to the geometry of the selected laparoscopic instrument and trocar, range measurements cannot exceed $23 \mathrm{~cm}$ and the resolution in the working volume of the training box was found to be better than $0.1 \mathrm{~cm}$ per LSB.

Combining the Sharp IR sensor with the Phidgets IMU completes the hardware design of a system capable of tracking orientation and position. Figure 3.4 shows an implementation of the sensor-augmented laparoscopic grasping instrument. It is worth noting that the IR sensor is capable of useful distance sensing without the use of a reflective disc but due to the contoured head of the trocar, reliable millimetre accuracy was only possible once the disc was attached. Used to simulate tissue elasticity, a silicone insert has also been included, as this represents the point 


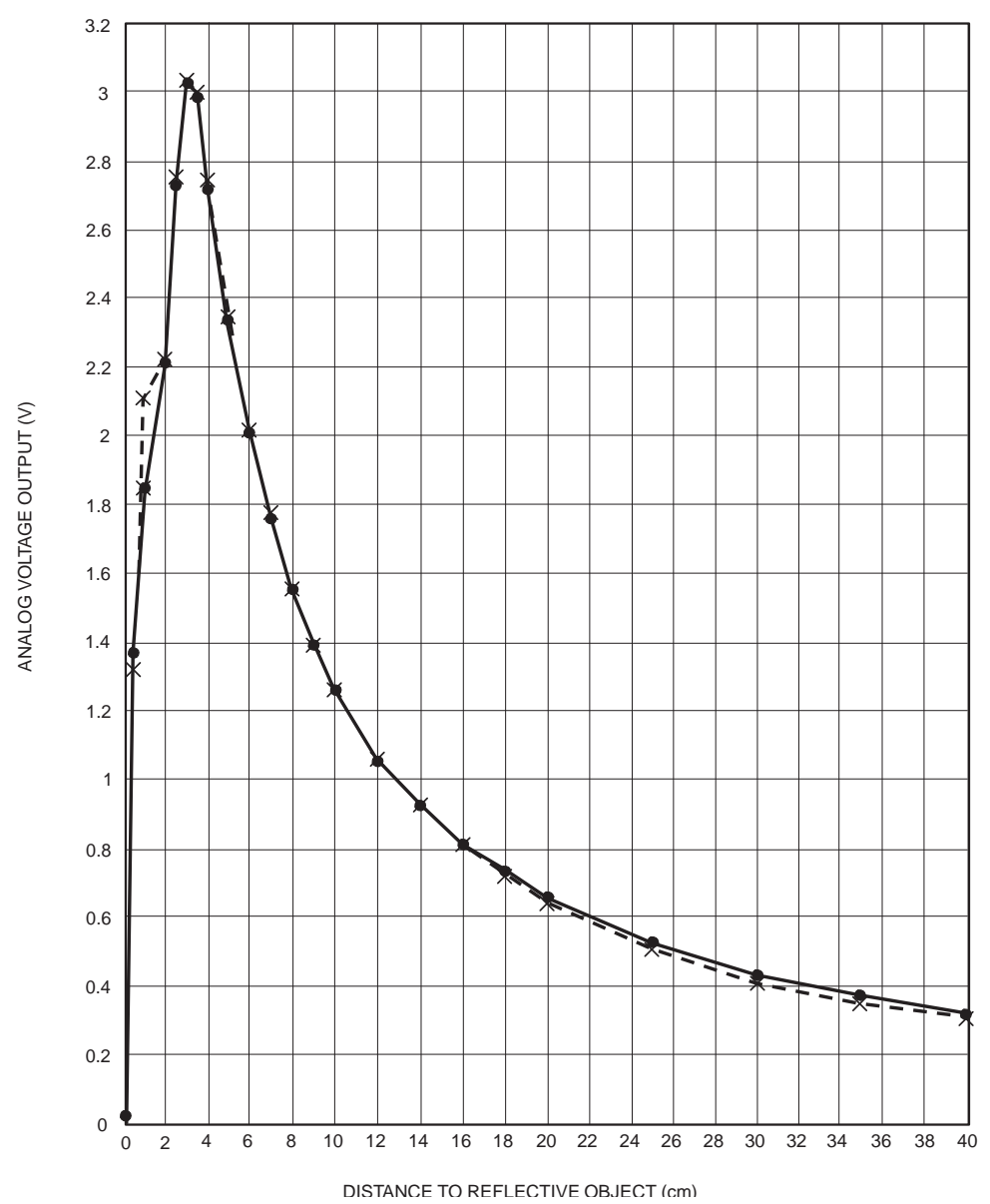

Figure 3.3: Sharp GP2D120 distance to voltage output characteristics.

about which the instrument is expected to pivot. Figure 3.5 shows the instrument operating in the simulated environment of a laparoscopic training box.

This device has been called the Inertial and Range-Enhanced Surgical (IRES) Tracker.

\subsection{Algorithm Development}

\subsubsection{Introduction and Goals}

Following sensor selection and hardware development, attention must be focused on software design. This tracking system relies on the fusion of multiple sensing modalities to deliver real-time tracking performance that is both accurate and reliable. To date, no research has been found that makes use of this combination of inertial and range sensing to track laparoscopic instruments. 


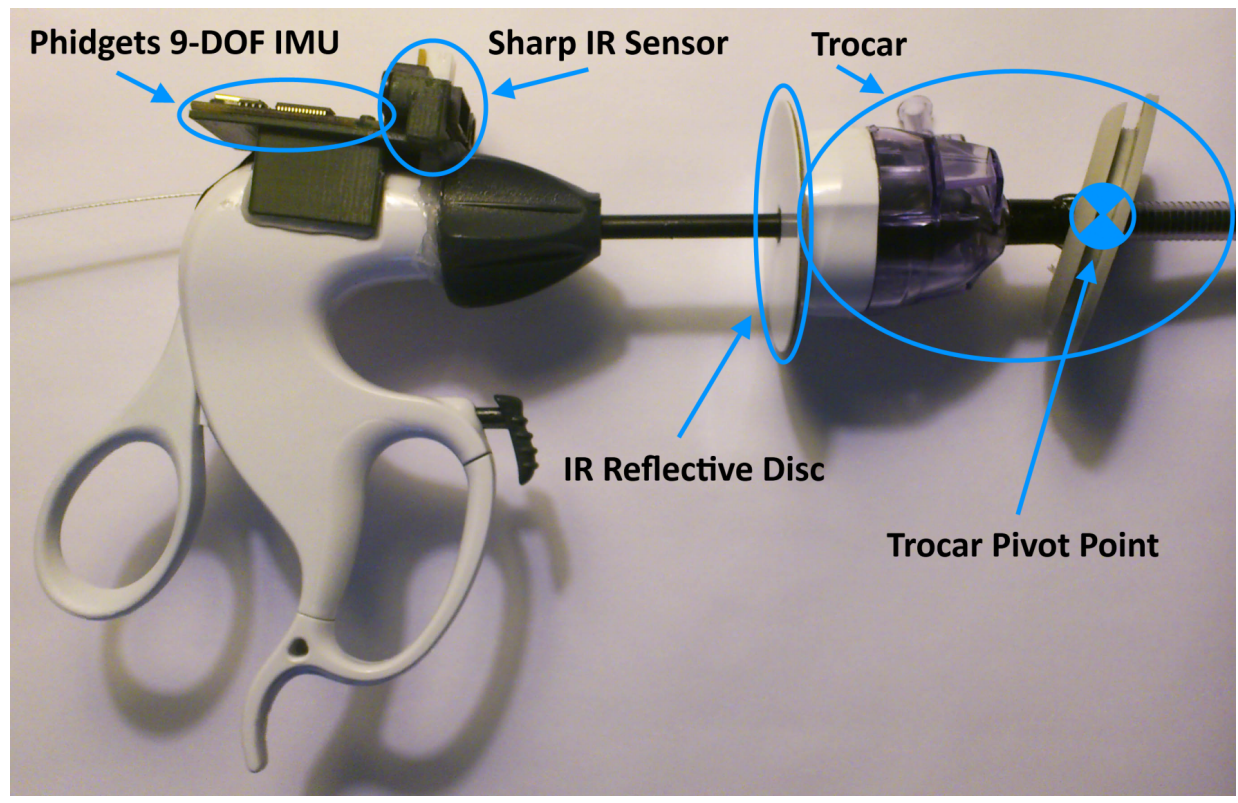

Figure 3.4: Laparoscopic instrument augmented with the IRES Tracker.

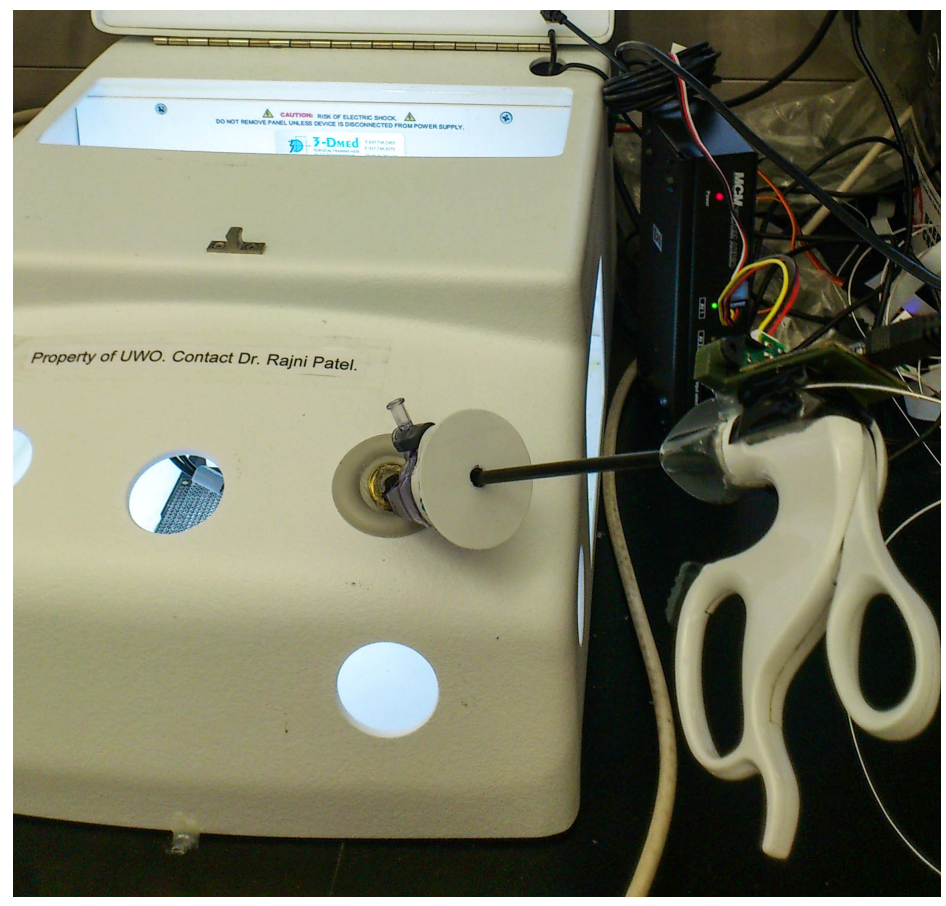

Figure 3.5: Laparoscopic instrument operating in a training box. 
While AHRS are widely found in applications such as head tracking and vehicle navigation, these systems tend to be ill-suited for medical tracking purposes where unstable magnetic fields and hand tremor may be present. Orientation sensing also represents an incomplete solution when full pose information is required. An algorithm must be developed to fuse inertial and distance sensing modalities to provide a complete laparoscopic tracking solution.

The main goal of the IRES tracking software is to provide continuous, real-time 6-DOF pose information (orientation and position). It should exhibit reasonable noise and interference rejection characteristics while maintaining an accuracy and speed comparable to that of modern electromagnetic tracking systems. If these goals can be achieved, the IRES tracker will be a viable alternative to existing motion tracking technologies.

\subsubsection{Euler Angles and Quaternions}

Before an investigation of sensor fusion algorithms can be discussed, it is important to look at the ways in which orientation is defined. The most common way to express the orientation of a rigid body is through the use of Euler angles comprising three rotations about the axes of a coordinate system in Euclidean space. These angles, typically defined as $\theta, \phi$ and $\psi$, can be expressed in radians or degrees. In body orientation and navigation situations, these angles are more commonly expressed as roll $(\theta)$, pitch $(\phi)$ and yaw $(\psi)$. Figure 3.6 follows the right-hand convention for defining positive roll, pitch and yaw.

A stationary accelerometer can be used to calculate roll and pitch angles by measuring the components of gravitational acceleration acting along each axis $\left(g_{\mathrm{x}}, g_{\mathrm{y}}, g_{\mathrm{z}}\right)$ as shown in Equations 3.4 and 3.5 :

$$
\begin{gathered}
\theta_{\text {roll }}=\tan ^{-1}\left(\frac{-g_{\mathrm{x}}}{g_{\mathrm{z}}}\right), \text { where }-\pi \leq \theta_{\text {roll }} \leq \pi, \\
\phi_{\text {pitch }}=\tan ^{-1}\left(\frac{g_{\mathrm{y}}}{\sqrt{g_{\mathrm{x}}^{2}+g_{\mathrm{z}}^{2}}}\right), \text { where }-\frac{\pi}{2} \leq \phi_{\text {pitch }} \leq \frac{\pi}{2},
\end{gathered}
$$

From here, magnetometer readings $\left(m_{\mathrm{x}}, m_{\mathrm{y}}, m_{\mathrm{z}}\right)$ can be tilt-compensated by inversely applying the roll and pitch angles to rotate the magnetometer into the local level, wander azimuth frame 


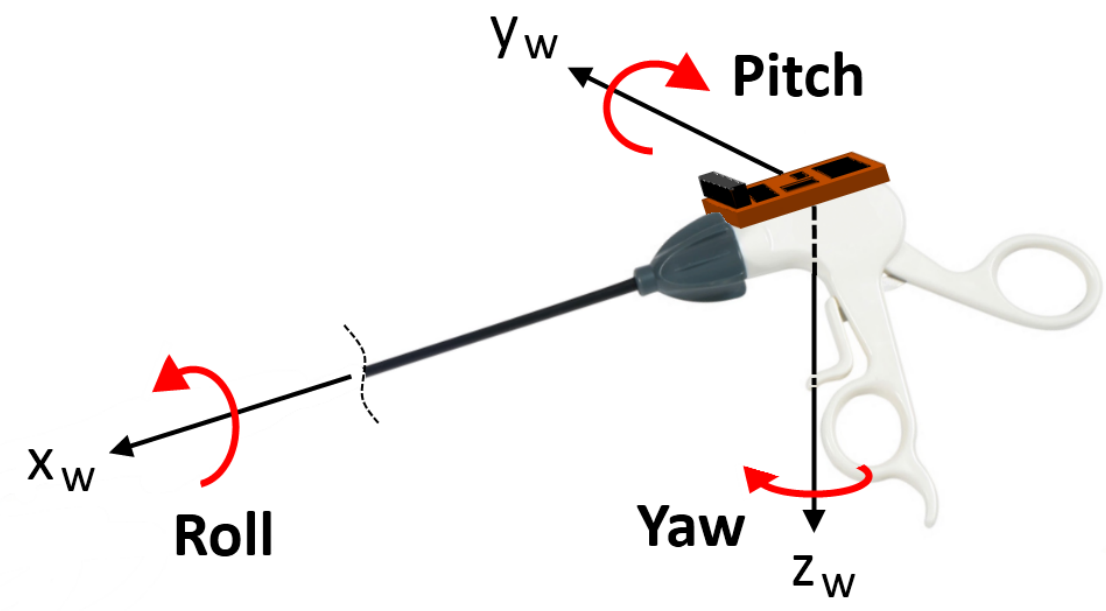

Figure 3.6: Right-handed coordinate system defining Euler angles.

( $L$ frame). The components of the yaw angle can then be calculated using Equations 3.6 and 3.7 and the yaw angle can be determined from Equation 3.8:

$$
\begin{gathered}
h_{\mathrm{x}}=m_{\mathrm{x}} \cos \left(\phi_{\text {pitch }}\right)+m_{\mathrm{z}} \sin \left(\phi_{\text {pitch }}\right), \\
h_{\mathrm{y}}=m_{\mathrm{x}} \sin \left(\theta_{\text {roll }}\right) \sin \left(\phi_{\text {pitch }}\right)+m_{\mathrm{y}} \cos \left(\theta_{\text {roll }}\right)-m_{\mathrm{z}} \sin \left(\theta_{\text {roll }}\right) \cos \left(\phi_{\text {pitch }}\right), \\
\psi_{\text {yaw }}=\tan ^{-1}\left(\frac{h_{\mathrm{y}}}{h_{\mathrm{x}}}\right), \text { where }-\pi \leq \psi_{\text {yaw }} \leq \pi .
\end{gathered}
$$

While the use of Euler angles is elegant in its simplicity, this method does not provide a continuous solution across the entire computational domain. At roll angles of $\pm \frac{\pi}{2}, g_{\mathrm{z}}$ approaches zero, resulting in an undefined condition in Equation 3.4 known as gimbal lock. Similarly, at pitch angles of $\pm \frac{\pi}{2}$, the denominator of Equation 3.5 approaches zero leading to a similarly undefined condition. For Eulerian AHRS, these singularities often cause the algorithm to freeze or crash unless the software is designed to handle division by zero, in which case, orientation estimates will become highly erratic and unreliable. Since a laparoscopic instrument cannot be restricted in its range of motion, singularities are likely to occur when using an Eulerian approach and another 
method must be adopted.

Quaternions Quaternions are a mathematical notation used to represent singularity-free orientation and rotation. A quaternion is a four-parameter representation comprised of a vector, $\left[q_{2}, q_{3}, q_{4}\right]$, and the magnitude of rotation about this vector, $q_{1}$, as shown in Figure 3.7. It can be used to represent orientation and rotation in the form $\mathbf{q}=q_{1}+q_{2} \mathrm{i}+q_{3} \mathrm{j}+q_{4} \mathrm{k}$ where $q_{1}$ is the real component and $\left[q_{2}, q_{3}, q_{4}\right]$ is the imaginary component.

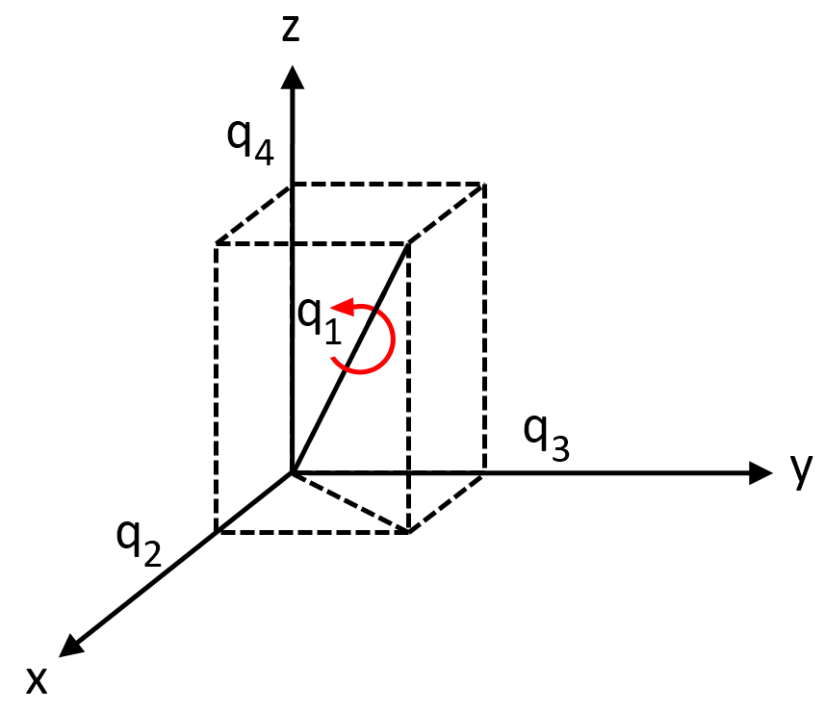

Figure 3.7: Four quaternion parameters representing orientation in 3-space.

A unit quaternion has a length of 1 and every unit quaternion represents a 3-dimensional rotation. Two consecutive rotation quaternions can be combined into a single equivalent quaternion by applying quaternion multiplication $\mathbf{q}^{\prime}=\mathbf{q}_{\mathbf{2}} \mathbf{q}_{\mathbf{1}}$, where $\mathbf{q}^{\prime}$ corresponds to the rotation of $\mathbf{q}_{\mathbf{1}}$ followed by $\mathbf{q}_{\mathbf{2}}$. Note that quaternion multiplication is non-commutative, meaning $\mathbf{q}_{2} \mathbf{q}_{\mathbf{1}} \neq \mathbf{q}_{\mathbf{1}} \mathbf{q}_{\mathbf{2}}$.

Quaternions can also be used to rotate an arbitrary vector $\mathbf{v}=\left[v_{\mathrm{x}}, v_{\mathrm{y}}, v_{\mathrm{z}}\right]$ by applying the quaternion rotation and its conjugate of the form $\mathbf{v}^{\prime}=\mathbf{q v q ^ { * }}$. The quaternion conjugate is defined as $\mathbf{q}^{*}=q_{1}-q_{2} \mathrm{i}-q_{3} \mathrm{j}-q_{4} \mathrm{k}$ and, for a unit quaternion, this is equal to its inverse as shown in Equation 3.9:

$$
\mathbf{q}^{-\mathbf{1}}=\frac{\mathbf{q}^{*}}{|\mathbf{q}|^{2}}=\mathbf{q}^{*}(\text { if }|\mathbf{q}|=1)
$$


The quaternion inverse makes it possible to divide quaternions and, with these basic relationships established, a quaternion-based AHRS can be developed. Quaternion-based approaches can often be less computationally demanding than equivalent Eulerian methods since only basic mathematical operations are used and no trigonometric functions are required.

\subsubsection{AHRS Sensor Fusion Algorithms}

As discussed in Section 3.2.2, an accelerometer and magnetometer-based AHRS is highly susceptible to noise and interference from inertial and magnetic sources, requiring the use of gyroscopes to provide accurate measurements. By updating the orientation estimate on the basis of integrated gyroscope input, the system becomes far less prone to interference. By feeding input from the accelerometers and magnetometers into the filtering algorithm, the system can provide a drift-free orientation estimate. In this section, the manner by which orientation estimates from these sensing modalities are fused is discussed.

\subsubsection{Complementary Filter}

One of the simplest AHRS sensor fusion algorithms is known as the complementary filter. In this filter, weight factors which sums to 1 are applied to the gyro and accelerometer/magnetometer inputs in order to govern how strongly the orientation estimate relies on each source for computing orientation at the current time step.

A quaternion representation of gyro angular rates can be constructed as shown in Equation 3.10 , where $\mathbf{q}_{\mathbf{t}-\mathbf{1}}$ is the quaternion estimate from the previous iteration and $\omega_{t}=\left[0, \omega_{\mathbf{x}}, \omega_{\mathrm{y}}, \omega_{\mathbf{z}}\right]^{\mathrm{T}}$ is a vector of gyro angular rates at the current time step. The gyro-predicted orientation quaternion can then be defined by Equation 3.11, where $\delta t$ is the elapsed time since the last iteration.

$$
\begin{gathered}
\dot{\mathbf{q}}_{t}=\frac{1}{2} \mathbf{q}_{t-1} * \omega_{t} \\
\mathbf{q}_{t \mid t-1}=\mathbf{q}_{t-1}+\dot{\mathbf{q}}_{t} \cdot \delta t
\end{gathered}
$$

A second orientation quaternion can be constructed from accelerometer and magnetometer 
data by solving an optimization problem [31] [32]. The most common approaches make use of the Gradient Descent method [33], the Gauss-Newton method [34] and the QUEST (QUaternion ESTimator) algorithm [35]. The QUEST algorithm, in particular, is an approach to orientation quaternion estimation from accelerometer and magnetometer data that avoids the computational cost of trigonometric functions and is non-iterative, unlike the Gradient Descent and Gauss-Newton methods. Furthermore, a singularity avoidance method introduced by Yun et al. [35] allows the QUEST algorithm to track through all orientations, thereby providing a more stable orientation estimate.

Once the predicted quaternion, $\mathbf{q}_{t \mid t-1}$, and measurement quaternion, $\mathbf{q}_{t}$, has been constructed, the complementary filter can be used to create a fused estimate using Equation 3.12, where $w_{\mathrm{cf}}$ is the filter gain, $0 \leq w_{\mathrm{cf}} \leq 1$. The resulting updated quaternion can then be fed into the next iteration of the complementary filter.

$$
\hat{\mathbf{q}}_{t}=w_{\mathrm{cf}} \cdot \mathbf{q}_{t \mid t-1}+\left(1-w_{\mathrm{cf}}\right) \cdot \mathbf{q}_{t}
$$

Since the complementary filter only requires the previously computed orientation estimate, this filter is ideal for embedded applications with limited memory and continues to be one of the most popular algorithms for orientation-based inertial sensing where speed and efficiency are critical.

\subsubsection{Linear Kalman Filter}

The complementary filter is a fixed-gain filter and does not consider what effect noise has on signal corruption. If two or more signals are fed into a complementary filter, the output will be a fixedratio combination of the input signals. By contrast, a Kalman filter considers the noise statistics of these input signals and can update the weight factors in an optimal manner to significantly improve filter performance [36]. The Kalman filter is one of the more popular approaches to AHRS that considers the statistics of signals corrupted by noise.

Named after Rudolf Kalman, the Linear Kalman Filter (LKF) uses a series of noisy measurements, often in the time domain, to estimate unknown variables with greater precision than can 
be achieved from single measurements. The Kalman filter is a two-step recursive filter that uses noisy input signals to estimate the underlying system state in a statically optimal manner. In the first step, a process model is used to generate a prediction of the current state vector and estimate the reliability of this prediction. In the second step, an observation is made of the underlying system state that has been corrupted by unknown error and noise. The prediction and observation uncertainties are compared and used to compute weight factors that favour the more reliable estimate. This statistical approach to computing filter gain results in a more accurate estimate of the underlying system.

A general form of the linear Kalman filter will now be presented in more detail.

Prediction Step The linear Kalman filter relies on a linear process model that mathematically defines the evolution of the state based on the true underlying process. This model is defined as:

$$
\vec{x}_{t \mid t-1}=\mathbf{F}_{t} \vec{x}_{t-1 \mid t-1}+\mathbf{B}_{t} u_{t}
$$

where the a priori state estimate, $\vec{x}_{t \mid t-1}$, is a prediction based on the previous state, $\vec{x}_{t-1 \mid t-1}$, and some new information introduced by the control vector, $u_{t} . \mathbf{F}_{t}$ is the state transition model and $\mathbf{B}_{t}$ is the control-input model (used to extract information from the control vector in the appropriate form for the linear model). Note that, not all Kalman filter implementations make use of a control-input model.

In addition to state prediction, an estimate must also be made about the prediction covariance:

$$
\mathbf{P}_{t \mid t-1}=\mathbf{F}_{t} \mathbf{P}_{t-1 \mid t-1} \mathbf{F}_{t}^{\mathrm{T}}+\mathbf{Q}_{t}
$$

The a priori state covariance, $\mathbf{P}_{t \mid t-1}$, is based on the updated covariance from the previous iteration and the addition of a process noise term, $\mathbf{Q}_{t}$. With the a priori state estimate and covariance estimate, the algorithm may evolve in one of two ways. If measurement input is rare or unpredictable, the filter may continue evolving based on the prediction model or if measurement input is available, an update may be performed. In this manner, the two stages of this algorithm may be run asynchronously. 
Update Step In order for the Kalman filter to correct for errors in the prediction model, an observation or measurement of the true underlying process must be made. The difference between the a priori state estimate and this observation is known as the innovation, $\tilde{y}_{t}$, as shown in Equation 3.15. $\mathbf{H}_{t}$ is the observation model which maps the state vector into the observation space:

$$
\tilde{y}_{t}=\overrightarrow{z_{t}}-\mathbf{H}_{t} \vec{x}_{t \mid t-1}
$$

Similarly, the innovation covariance can be determined as a sum of the predicted covariance and measurement noise, $\mathbf{R}$. Covariance is simply a measure of reliability of the system state; as such, the innovation covariance, $\mathbf{S}_{t}$, is a measure of reliability of the innovation:

$$
\mathbf{S}_{t}=\mathbf{H}_{t} \mathbf{P}_{t \mid t-1} \mathbf{H}_{t}^{\mathrm{T}}+\mathbf{R}
$$

With the innovation covariance and prediction covariance, the Kalman gain, $\mathbf{K}_{t}$, can be determined using Equation 3.17:

$$
\mathbf{K}_{t}=\mathbf{P}_{t \mid t-1} \mathbf{H}_{t}^{\mathrm{T}} \mathbf{S}_{t}^{-1}
$$

The Kalman gain is used to control how much innovation is introduced into the a posteriori state estimate. If the innovation covariance is high, the measurement is noisy and the Kalman gain will rely more heavily on the a priori estimate from the process model. The updated a posteriori state estimate can be calculated using Equation 3.18:

$$
\vec{x}_{t \mid t}=\vec{x}_{t \mid t-1}-\mathbf{K}_{t} \tilde{y}_{t}
$$

The updated state estimate represents the desired Kalman filter. The updated covariance matrix, $\mathbf{P}_{t \mid t}$, can be determined and used as input to the next iteration: 


$$
\mathbf{P}_{t \mid t}=\left(I-\mathbf{K}_{t} \mathbf{H}_{t}\right) \mathbf{P}_{t \mid t-1},
$$

where, $I$ is the identity matrix. When the filter moves to the next iteration, $\mathbf{P}_{t \mid t}$ becomes $\mathbf{P}_{t-1 \mid t-1}$ and is used to calculate the new prediction covariance, $\mathbf{P}_{t \mid t-1}$.

Since the prediction and update steps can be performed asynchronously, the state may continue to evolve based on prediction. This allows for continuous orientation estimates even if measurement updates are rare or slow due to computational burden.

\subsubsection{Model Validation}

If the linear process model is accurate and the process and measurement variances are zero mean with a Gaussian distribution, the updated state estimate, $\vec{x}_{t \mid t}$ can be expected to match the predicted state, $\vec{x}_{t \mid t-1}$. In this case, the innovation, $\tilde{y}_{t}$, should be zero and the expectation (E) can be defined as:

$$
\mathbf{E}\left[\vec{x}_{t}-\vec{x}_{t \mid t}\right]=\mathbf{E}\left[\vec{x}_{t}-\vec{x}_{t \mid t-1}\right]=0
$$

$$
\mathbf{E}\left[\tilde{y}_{t}\right]=0
$$

Observing the difference between the predicted and updated states can therefore give a measure of how well the process model is functioning for tracking the system.

\subsubsection{Nonlinear Kalman Filter}

Kalman's first work [37] involved a filter designed for linear dynamic systems where input signal variance followed a zero-mean and Gaussian distribution. Since an underlying process may often be nonlinear, filter tracking may use nonlinear process and observation models. For this reason, extensions of the Kalman filter were devised. The two most common Kalman filters for nonlinear systems are the Extended Kalman Filter (EKF) and the Unscented Kalman Filter (UKF). These 


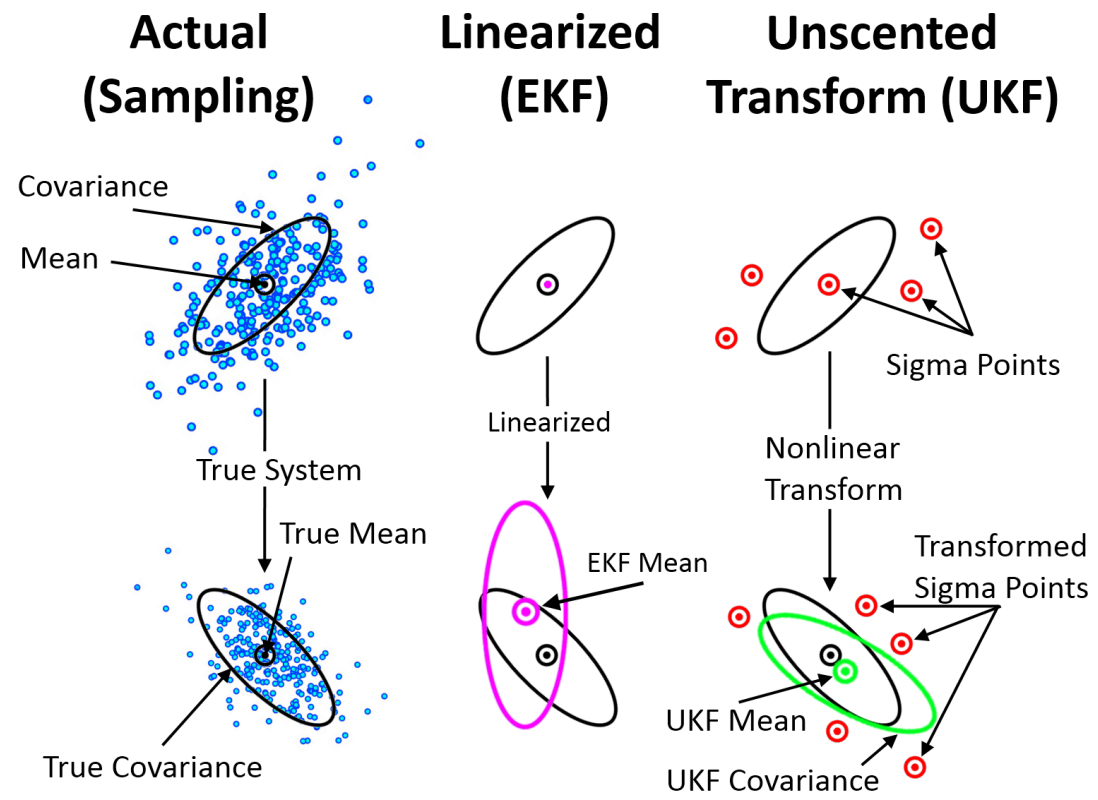

Figure 3.8: Visual representation of the extended and unscented Kalman filters.

filters are able to track second-order and higher statistical moments of a distribution resulting in more accurate state estimates for nonlinear processes.

The EKF operates by linearizing the nonlinear models through the use of Jacobians and a transition model. Once linearized, the filter can operate in a manner similar to the linear Kalman filter. The process of linearization is non-trivial and the transition models must be well defined. This often means an EKF is customized for a specific application and large, unexpected input deviations can cause the filter to become unstable. Nonetheless, the EKF remains popular due to its efficiency and performance with nonlinear tracking.

First introduced in 2004 [38], the Unscented Kalman Filter was designed to overcome the limitations of the EKF by eliminating the need to calculate Jacobians and define transition models. By defining a set of sigma points distributed about the mean of the state vector and propagating these points through the nonlinear models, the resulting distribution can be analyzed to recover the statistics of the process. In this manner, second-order (and higher) statistical moments of an undefined process can be recovered and used to update the Kalman gain. The single biggest advantage of the UKF is its ability to operate directly on a nonlinear system without the setup and customization required for the EKF. It has been found that the UKF is also capable of faster 
convergence and greater stability over the EKF for navigation and orientation-sensing applications [39].

The biggest drawback of the UKF is computational load. For an $n$-dimensional state vector, the standard UKF requires the use of $2 n+1$ sigma points. This means that instead of running the process model once for each iteration as with the LKF, the models must be rerun for each point and the computational load increases by a factor of $2 n+1$. For real-time tracking performance using current-generation computer hardware, the computational demand of the UKF can often be a limiting factor.

Many inertial systems are based on nonlinear dynamic models that make them best suited for a nonlinear filtering algorithm. Furthermore, the use of quaternions often requires a nonlinear approach since operations such as quaternion multiplication can cause the state vector to evolve in a nonlinear fashion.

Applying the UKF to an INS and AHRS During a phase of this research, a UKF was designed and implemented to track not only position, velocity and orientation of a MEMS-based INS, but also sensor calibration parameters such as zero offsets, scaling factors and magnetometer calibration parameters. The aim of this phase of research was to create a filter capable of tracking the dominant sources of error inherent to lower-grade MEMS devices with the goal of improving sensor performance for INS applications. This area of research was based on the work by Shin et al. [40], Grewal et al. [41] and Crassidis et al. [42].

The implementation of this UKF-based algorithm required the use of a 38-element state vector. With $2 n+1$ sigma points, this meant performing the Cholesky decomposition of matrices with several thousand elements for each iteration. Running this filter as a MATLAB script on an Intel Core i5 $750 \mathrm{CPU}$ (MATLAB single-core support) resulted in the computation of 4 samples per second. By switching to the Spherical Simplex Unscented Transform [43], the number of sigma points could be reduced to $n+2$, bringing computational performance up to around 7 iterations per second. Since a typical INS requires performance upwards of 1000 iterations per second, this approach was abandoned. It was determined that computational complexity exceeded currentgeneration hardware capabilities. 
Though a UKF-based INS was found to be impractical, use of the UKF for a quaternion-based AHRS presented a much simpler design goal and it was expected that such a system could be capable of real-time performance.

The result of this UKF-based AHRS implementation was a system capable of 23 samples per second on the same Intel Core i5 750 CPU. While this represented a significant step toward acceptable real-time performance, computational requirements remained non-trivial. It was determined that what little orientation tracking improvement could be achieved by nonlinear filtering could not be justified when compared with the computational demand. A simpler filtering method was required.

\subsubsection{A Quaternion-Based Linear Kalman Filter for Orientation Sensing}

Many quaternion-based AHRS algorithms require the use of nonlinear filtering; however, the computational complexity of nonlinear Kalman-based methods proved to be more demanding than desired. Considering the developed system should ultimately be capable of deployment on embedded devices, a greater emphasis was placed on filter efficiency. The LKF provided a balance between computational efficiency and robust tracking performance.

The challenge became the design of a linear, quaternion-based process model for use with the LKF. Based on the work of Comotti et al. [36], one such algorithm was developed.

The simplest approach is to define a state vector as four elements of a quaternion to track the orientation of the sensing platform. The state vector can be defined as follows:

$$
\vec{x}=\left[\begin{array}{l}
q_{1} \\
q_{2} \\
q_{3} \\
q_{4}
\end{array}\right] .
$$

From here, the operation of the filter is divided into two stages, as discussed in Section 3.3.3.2.

1. Prediction Step: Evolution of the state vector from the previous update is predicted for the current time step using a process model known as the state evolution matrix, F. Integrated 
angular rates from the gyros are used to generate this a priori estimate.

2. Update Step: An observation, $\vec{z}_{t}$, is used to update the a priori state estimate by calculating the covariance between the predicted state and the observation and applying the Kalman gain to generate an updated a posteriori estimate. The measurement is captured from accelerometer and magnetometer inputs, which provide a noisy observation of the underlying system. Finally, the updated covariance is calculated and passed along with the updated state vector into the next iteration of the filter.

Prediction Implementation As shown in Equation 3.10, angular velocity input can be converted into quaternion form:

$$
\dot{q}_{t}=\frac{1}{2} \cdot \vec{x}_{t-1 \mid t-1} \otimes \vec{\omega}
$$

Integrating 3.23 using the iteration time step, $\delta t$, provides a rotation quaternion that can be used to generate the a priori state estimate, $\vec{x}_{t \mid t-1}$ :

$$
\vec{x}_{t \mid t-1}=\vec{x}_{t-1 \mid t-1}+\dot{q}_{t} \cdot \delta t=\mathbf{F}_{t} \cdot \vec{x}_{t-1 \mid t-1} \cdot
$$

The state transition model, $\mathbf{F}_{t}$, is a combined form of Equation 3.23 and 3.24, which uses gyro sensor inputs to evolve the state prediction:

$$
\mathbf{F}_{t}=\left[\begin{array}{cccc}
1 & -\frac{1}{2} \cdot \omega_{\mathrm{x}} \cdot \delta t & -\frac{1}{2} \cdot \omega_{\mathrm{y}} \cdot \delta t & -\frac{1}{2} \cdot \omega_{\mathrm{z}} \cdot \delta t \\
\frac{1}{2} \cdot \omega_{\mathrm{x}} \cdot \delta t & 1 & \frac{1}{2} \cdot \omega_{\mathrm{z}} \cdot \delta t & -\frac{1}{2} \cdot \omega_{\mathrm{y}} \cdot \delta t \\
\frac{1}{2} \cdot \omega_{\mathrm{y}} \cdot \delta t & -\frac{1}{2} \cdot \omega_{\mathrm{z}} \cdot \delta t & 1 & \frac{1}{2} \cdot \omega_{\mathrm{z}} \cdot \delta t \\
\frac{1}{2} \cdot \omega_{\mathrm{z}} \cdot \delta t & \frac{1}{2} \cdot \omega_{\mathrm{y}} \cdot \delta t & -\frac{1}{2} \cdot \omega_{\mathrm{x}} \cdot \delta t & 1
\end{array}\right]
$$


To create a model of the process noise, $\vec{w}$, the variance of static signals along each gyro axis can be used to create the process covariance matrix, $\mathbf{Q}_{t}$, as shown in Equation 3.26:

$$
\mathbf{Q}_{t}=\mathbf{E}\left[\left[\begin{array}{c}
-\omega_{\mathrm{x}}-\omega_{\mathrm{y}}-\omega_{\mathrm{z}} \\
\omega_{\mathrm{x}}-\omega_{\mathrm{y}}+\omega_{\mathrm{z}} \\
\omega_{\mathrm{x}}+\omega_{\mathrm{y}}-\omega_{\mathrm{z}} \\
-\omega_{\mathrm{x}}+\omega_{\mathrm{y}}+\omega_{\mathrm{z}}
\end{array}\right] \cdot\left[\begin{array}{c}
-\omega_{\mathrm{x}}-\omega_{\mathrm{y}}-\omega_{\mathrm{z}} \\
\omega_{\mathrm{x}}-\omega_{\mathrm{y}}+\omega_{\mathrm{z}} \\
\omega_{\mathrm{x}}+\omega_{\mathrm{y}}-\omega_{\mathrm{z}}^{\mathrm{T}} \\
-\omega_{\mathrm{x}}+\omega_{\mathrm{y}}+\omega_{\mathrm{z}}
\end{array}\right]\right]^{\mathrm{s}}
$$

Assuming that the gyro offsets have been corrected or are negligible, the expected variance of a gyro signal should be $\mathbf{E}\left[\omega_{i}\right]=0$. Between axes, it should likewise be $\mathbf{E}\left[\omega_{i} \cdot \omega_{j}\right]=0$. Process covariance can therefore be updated to reflect the signal variance along each axis:

$$
\mathbf{Q}_{t}=\left[\begin{array}{cccc}
\sigma_{\mathrm{x}}^{2}+\sigma_{\mathrm{y}}^{2}+\sigma_{\mathrm{z}}^{2} & -\sigma_{\mathrm{x}}^{2}+\sigma_{\mathrm{y}}^{2}-\sigma_{\mathrm{z}}^{2} & -\sigma_{\mathrm{x}}^{2}-\sigma_{\mathrm{y}}^{2}+\sigma_{\mathrm{z}}^{2} & \sigma_{\mathrm{x}}^{2}-\sigma_{\mathrm{y}}^{2}-\sigma_{\mathrm{z}}^{2} \\
-\sigma_{\mathrm{x}}^{2}+\sigma_{\mathrm{y}}^{2}-\sigma_{\mathrm{z}}^{2} & \sigma_{\mathrm{x}}^{2}+\sigma_{\mathrm{y}}^{2}+\sigma_{\mathrm{z}}^{2} & \sigma_{\mathrm{x}}^{2}-\sigma_{\mathrm{y}}^{2}-\sigma_{\mathrm{z}}^{2} & -\sigma_{\mathrm{x}}^{2}-\sigma_{\mathrm{y}}^{2}+\sigma_{\mathrm{z}}^{2} \\
-\sigma_{\mathrm{x}}^{2}-\sigma_{\mathrm{y}}^{2}+\sigma_{\mathrm{z}}^{2} & \sigma_{\mathrm{x}}^{2}-\sigma_{\mathrm{y}}^{2}-\sigma_{\mathrm{z}}^{2} & \sigma_{\mathrm{x}}^{2}+\sigma_{\mathrm{y}}^{2}+\sigma_{\mathrm{z}}^{2} & -\sigma_{\mathrm{x}}^{2}+\sigma_{\mathrm{y}}^{2}-\sigma_{\mathrm{z}}^{2} \\
\sigma_{\mathrm{x}}^{2}-\sigma_{\mathrm{y}}^{2}-\sigma_{\mathrm{z}}^{2} & -\sigma_{\mathrm{x}}^{2}-\sigma_{\mathrm{y}}^{2}+\sigma_{\mathrm{z}}^{2} & -\sigma_{\mathrm{x}}^{2}+\sigma_{\mathrm{y}}^{2}-\sigma_{\mathrm{z}}^{2} & \sigma_{\mathrm{x}}^{2}+\sigma_{\mathrm{y}}^{2}+\sigma_{\mathrm{z}}^{2}
\end{array}\right]
$$

The a priori state estimate, $\vec{x}_{t \mid t-1}$, can be defined using Equation 3.28 , where $\vec{x}_{t-1 \mid t-1}$ is the a posteriori estimate from the previous filter iteration:

$$
\vec{x}_{t \mid t-1}=\mathbf{F}_{t} \cdot \vec{x}_{t-1 \mid t-1} .
$$

Finally, a measure of the prediction covariance, $\mathbf{P}_{t \mid t-1}$, is determined using Equation 3.29:

$$
\mathbf{P}_{t \mid t-1}=\left(\mathbf{F}_{t} \cdot \mathbf{Q}_{t} \cdot \mathbf{F}_{t}^{\mathrm{T}}\right)+\mathbf{Q}_{t}
$$


Generating an Observation In this filter, the observation, $\vec{z}_{t}$, consists of an orientation quaternion based on measurements from accelerometers and magnetometers. As described in the work by Madgwick [31] [32], the computation of an orientation quaternion using these sensors often becomes an optimization problem. To minimize error and generate this quaternion, the Gradient Descent method, Gauss-Newton method [36] or some formulation of the QUEST algorithm [35] are most commonly used. For simplicity, the Gradient Descent method was implemented to generate the observation.

Since this filter's state space and observation space are both quaternions representing the orientation of the sensing platform, the observation model, $\mathbf{H}_{t}$, which maps the state vector into the observation space is simply a time-invariant identity matrix. No conversion or remapping is required:

$$
\mathbf{H}=I_{4 \times 4} .
$$

Update Implementation In this stage, the predicted state vector, $\vec{x}_{t \mid t-1}$, is updated by input from the observation, after which the Kalman filter gain, $\mathbf{K}_{t}$, can be calculated by combining Equations 3.16 and 3.17 to produce Equation 3.31:

$$
\mathbf{K}_{t}=\mathbf{P}_{t \mid t-1} \cdot \mathbf{H} \cdot\left(\mathbf{H} \cdot \mathbf{P}_{t \mid t-1} \cdot \mathbf{H}^{\mathrm{T}}+\mathbf{R}\right)^{-1}
$$

Normally, the observation covariance matrix, $\mathbf{R}$, contains terms reflecting the variance of the observation signal. In this case, the accelerometer and magnetometer variance is obfuscated by the method used to generate the observation quaternion and $\mathbf{R}$ must be tuned manually. $\mathbf{R}$ is often represented by an identity matrix multiplied by a constant, where $w_{\mathrm{R}}=0.001$ is a good starting parameter:

$$
\mathbf{R}=I_{4 \times 4} \cdot w_{\mathrm{R}} .
$$


The advantage of manually selecting $w_{\mathrm{R}}$ is that filter performance can be tuned for greater noise rejection from the observation model. If vibration or oscillating magnetic fields are causing interference, increasing $w_{\mathrm{R}}$ tells the filter that the observation signal contains greater variance and should be considered less reliable. The result is a more stable orientation estimate with the Kalman gain favouring the predicted state estimate.

Finally, the a posteriori state estimate, $\vec{x}_{t \mid t}$, can be computed by combining Equations 3.15 and 3.18 to give Equation, 3.33 where $\left(\vec{z}_{t}-\vec{x}_{t \mid t-1}\right)$ is the innovation between the observation and a priori estimate:

$$
\vec{x}_{t \mid t}=\vec{x}_{t \mid t-1}+\mathbf{K}_{t} \cdot\left(\vec{z}_{t}-\vec{x}_{t \mid t-1}\right)
$$

This completes the quaternion-based orientation tracking portion of the algorithm.

\subsubsection{Position Tracking}

Knowledge of instrument orientation can now be used to recover positional information at the tip of the instrument. To accomplish positional tracking, some measure of the axial distance of the instrument with respect to the pivot point of the trocar must be made. This distance tracking was achieved by using the SHARP GP2D120 4-30 cm IR sensor attached to the instrument handle with an optically reflective disk mounted to the face of the trocar. Since the IR sensor output exhibits an exponential relationship to distance, a linearizing equation must be derived based on experimentation. If the IR sensor signal is noisy, additional filtering may be applied to the signal by either modifying the Kalman filter to include an additional state parameter or by applying a simpler filtering method such as a moving average filter.

Since instrument orientation is used to project the length of the instrument from its pivot point to the tip in 3-D Cartesian coordinates, the geometry of the instrument is of critical importance to obtain accurate positional information. Referring to the instrument diagram in Figure 3.9, the total length of the instrument from IR sensor to tip is $l_{\mathrm{IR} \mid \mathrm{t} i}$, the length from the IR sensor to the 
reflective disk is $l_{\mathrm{IR} \mid \mathrm{di}}$ and the length from the reflective disk to the pivot point (remote centre of motion) of the trocar is $l_{\mathrm{di} \mid \mathrm{rp}}$. The desired length from the pivot point to the tip, $l_{\mathrm{rp} \mid \mathrm{ti}}$, can then be defined as:

$$
l_{\mathrm{rp} \mid \mathrm{ti}}=l_{\mathrm{IR} \mid \mathrm{ti}}-l_{\mathrm{IR} \mid \mathrm{di}}-l_{\mathrm{di} \mid \mathrm{rp}} .
$$

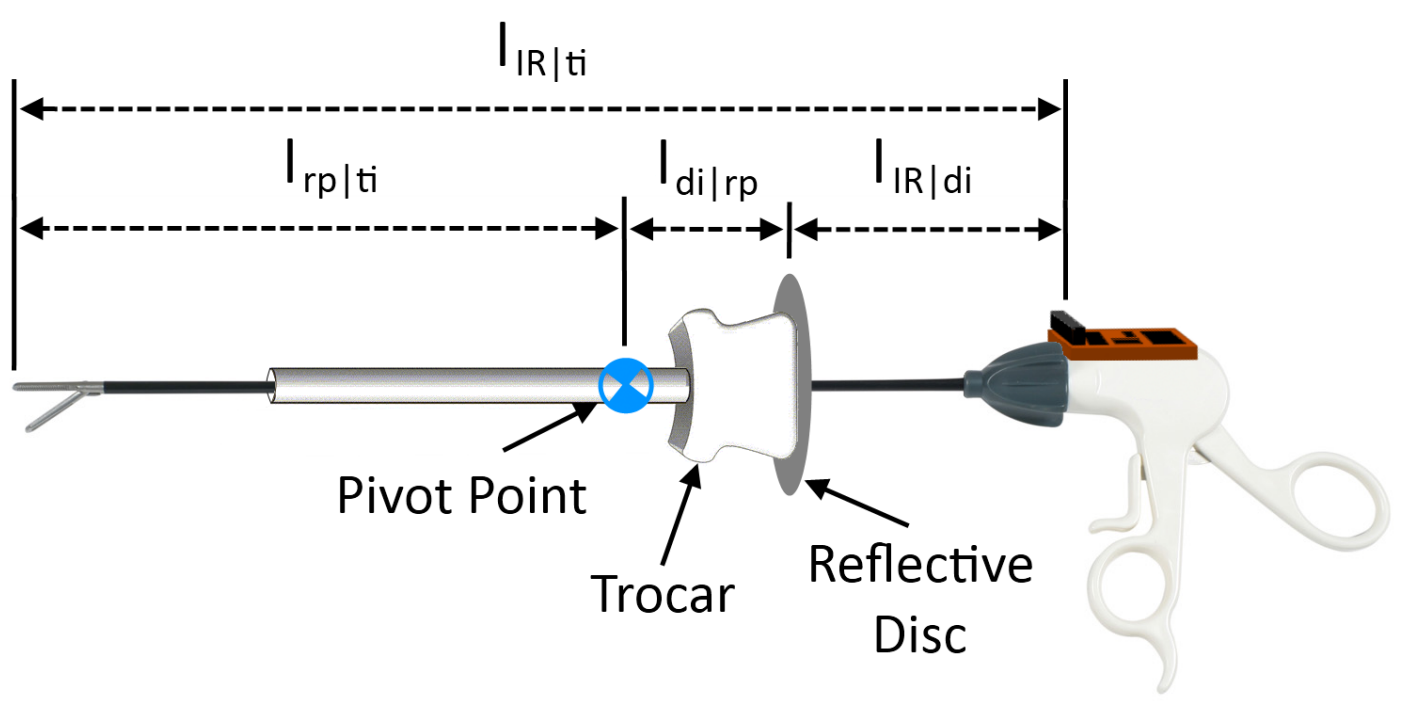

Figure 3.9: Diagram of system length variables for dimensional analysis.

For a rigid instrument and trocar, $\left(l_{\mathrm{IR} \mid \mathrm{ti}}-l_{\mathrm{di} \mid \mathrm{rp}}\right)$ is a constant. To initialize this algorithm, an approximation for $l_{\mathrm{IR} \mid \mathrm{ti}}$ and $l_{\mathrm{di} \mid \mathrm{rp}}$ must be made. If further calibration is performed to transform pose information from the IRES tracking frame into another frame and scaling is a parameter of the calibration routine, the accuracy of these instrument lengths is not critical. Calibration scaling will correct for measurement error at this stage.

By defining the instrument's pivot point as the origin of the IRES tracker $(0,0,0)$, the tip position can be mapped into Cartesian space by first converting the orientation quaternion $q_{t \mid t}=$ $\left[q_{1}, q_{2}, q_{3}, q_{4}\right]^{\mathrm{T}}$ into Euler angles using Equations 3.35 and 3.36, and then by applying Equations $3.38,3.39$ and 3.40 :

$$
\psi_{\text {yaw }}=\operatorname{atan} 2\left(\frac{2 \cdot\left(q_{1} \cdot q_{2}+q_{3} \cdot q_{4}\right)}{1-2 \cdot\left(q_{2} \cdot q_{2}+q_{3} \cdot q_{3}\right)}\right)
$$




$$
\begin{gathered}
\phi_{\text {pitch }}=\arcsin \left(2 \cdot\left(q_{1} \cdot q_{3}-q_{4} \cdot q_{2}\right)\right), \\
\theta_{\text {roll }}=\operatorname{atan} 2\left(\frac{2 \cdot\left(q_{1} \cdot q_{4}+q_{2} \cdot q_{3}\right)}{1-2 \cdot\left(q_{3} \cdot q_{3}+q_{4} \cdot q_{4}\right)}\right),
\end{gathered}
$$

$$
\begin{gathered}
x=l_{\mathrm{rp} \mid \mathrm{ti}} \cdot \sin \left(\psi_{\text {yaw }}\right) \cdot \cos \left(\phi_{\text {pitch }}\right), \\
y=-l_{\mathrm{rp} \mid \mathrm{ti}} \cdot \cos \left(\psi_{\text {yaw }}\right) \cdot \cos \left(\phi_{\text {pitch }}\right), \\
z=l_{\mathrm{rp} \mid \mathrm{ti}} \cdot \sin \left(\phi_{\text {pitch }}\right) .
\end{gathered}
$$

This concludes the IRES tracking algorithm designed to provide real-time 6-DOF orientation and position information.

\subsubsection{Calibration}

By default, the IRES tracker provides pose information in a coordinate frame that may need to be transformed into a second working frame. One of the easiest ways to determine this transform is to capture point pairs in both the source frame and the target frame and use these pairs to determine the transform. A method such as Horn's closed-form solution to the least squares problem [44] [45] can be implemented using as few as three pairs of points, making it a very robust solution for quick calibration. More points will produce a more accurate transformation. Horn's efficient quaternionbased method can solve for rotation, translation and optionally, the scaling that best maps the source data into the target frame.

Provided that the length of the instrument does not change, initialization of the tracking system using only approximated length values will not cause performance degradation when the calibration routine includes a scaling parameter. This reduces the time required to initialize the IRES tracker.

Once $n$ point pairs have been captured $(n \geq 3)$ and Horn's method has been used to determine the rigid transformation matrix, this transform can be returned to the IRES tracking algorithm 
so that pose information is correctly registered to the target environment.

\subsection{Summary}

In this chapter, the hardware and software design of an inertial and range-enhanced 6-DOF laparoscopic tracking system was presented. Hardware development involved using an AHRS to determine device orientation and an IR sensor to track the axial position of the instrument with respect to the trocar. Assuming that a point exists at the trocar-tissue interface where motion of the trocar is limited to rotation, AHRS orientation estimates can be used to project instrument depth into Cartesian coordinates.

Software development explored the use of complementary and Kalman-based AHRS algorithms for the fusion of accelerometer, gyroscope and magnetometer data. Linear and nonlinear Kalman filtering algorithms were discussed and the implementation of a quaternion-based linear Kalman filter was presented. Finally, the algorithm was augmented with distance information to calculate position at the instrument tip.

In the following chapter, performance of the IRES tracking system will be assessed and compared to an EM tracking system. 


\section{Chapter 4}

\section{Testing and Validation}

In this chapter, the performance of the IRES tracker is investigated and compared to an electromagnetic tracking system. Due to its high accuracy and the ability to operate without a line-of-sight, an electromagnetic tracker is considered the gold standard for medical instrument tracking, making it an ideal benchmark with which to test and validate the IRES tracking system.

\subsection{Testing Methodology}

Testing of the IRES and electromagnetic tracking systems follows an identical experimental method, based on a comparison of tip location estimates against an absolute reference. Pose information for each system is captured across 2-D planes stacked to create 3-D volumes of data. Accuracy, precision, repeatability, data spread, and other metrics are used to quantify and compare performance. An investigation of IRES tracker robustness in the presence of electromagnetic interference is also included.

The absolute reference system consists of a $2.00^{\prime \prime} \times 3.00^{\prime \prime}(50.8 \mathrm{~mm} \times 76.2 \mathrm{~mm})$ perforated board with $0.100^{\prime \prime} \pm 0.007^{\prime \prime}(2.54 \mathrm{~mm} \pm 0.18 \mathrm{~mm})$ hole spacing raised in $1.00^{\prime \prime}(25.4 \mathrm{~mm})$ increments using $1.00^{\prime \prime} \times 2.00^{\prime \prime} \times 3.00^{\prime \prime} \pm 0.001^{\prime \prime}(25.4 \mathrm{~mm} \times 50.8 \mathrm{~mm} \times 76.2 \mathrm{~mm} \pm 0.03 \mathrm{~mm})$ steel machinist blocks A pattern of 20 evenly spaced holes with a separation of $0.5^{\prime \prime}(12.7 \mathrm{~mm})$ on the perforated board defines the test plane. The machinist blocks raise the test plane to $1.00^{\prime \prime}$ (25.4 mm) and 2.00" (50.8 $\mathrm{mm}$ ) from the lower interior surface of the training box to produce 60 absolute reference locations. 
A tapered pin attached to the tip of the instrument is used to mechanically align the instrument to the centre of each hole, thereby ensuring accurate positioning at each location. Figure 4.1 shows the positive axes of the absolute reference system and placement of the system inside the training box. In this figure, the instrument tip is at location $\left(1.00^{\prime \prime}, 1.00^{\prime \prime}, 1.00^{\prime \prime}\right)$ or $(25.4 \mathrm{~mm}, 25.4 \mathrm{~mm}$, $25.4 \mathrm{~mm}$ ) in the absolute reference coordinate frame or working frame of the training box.

Three different cases were explored using 20, 40 and 60 points, based on the typical working volumes for laparoscopic procedures. A CMOS camera mounted inside of the training box simulates the FOV of a laparoscopic camera. Figure 4.2 shows a sample frame from this camera during a 20-point test representing planar XY tracking across the centre region of the image (approximately $40 \%$ of the viewable area). The 40-point case represents tracking within a volume of $82 \mathrm{~cm}^{3}$ that, covers $60 \%$ of the camera's FOV at $\mathrm{Z}=1.00^{\prime \prime}(\mathrm{Z}=25.4 \mathrm{~mm})$. At $164 \mathrm{~cm}^{3}$, the 60 -point case is so large that it exceeds the field of view of the camera.

Test planes are stacked in such a way that increasing height in the $\mathrm{Z}$ direction forces the instrument to swing through larger azimuth and elevation angles to reach every point. This is especially relevant for the IRES tracker, as it is expected that sharper instrument angles will cause greater deformation of the silicone pivot point. Since position estimation is based on the assumption that the pivot is a fixed datum, deformation at this point is expected to test this assumption.

\subsubsection{System Registration and Calibration}

IRES and electromagnetic tracking data must be transformed into the coordinate frame of the reference blocks before data analysis can be conducted. Position estimates at each reference point are collected and processed using Horn's method [44] to determine the rigid transformation (rotation, translation and scaling) that best registers each system to this common frame. Applying this transformation to all future system output completes the registration process.

In close proximity to lab equipment, power supplies and electromagnetic tracking systems, the local magnetic field around the IRES tracker may not align with magnetic north. This will offset orientation estimates from the $N$ frame; however, as long as the local magnetic field remains directionally stable, the system can be expected to function normally. This condition also 


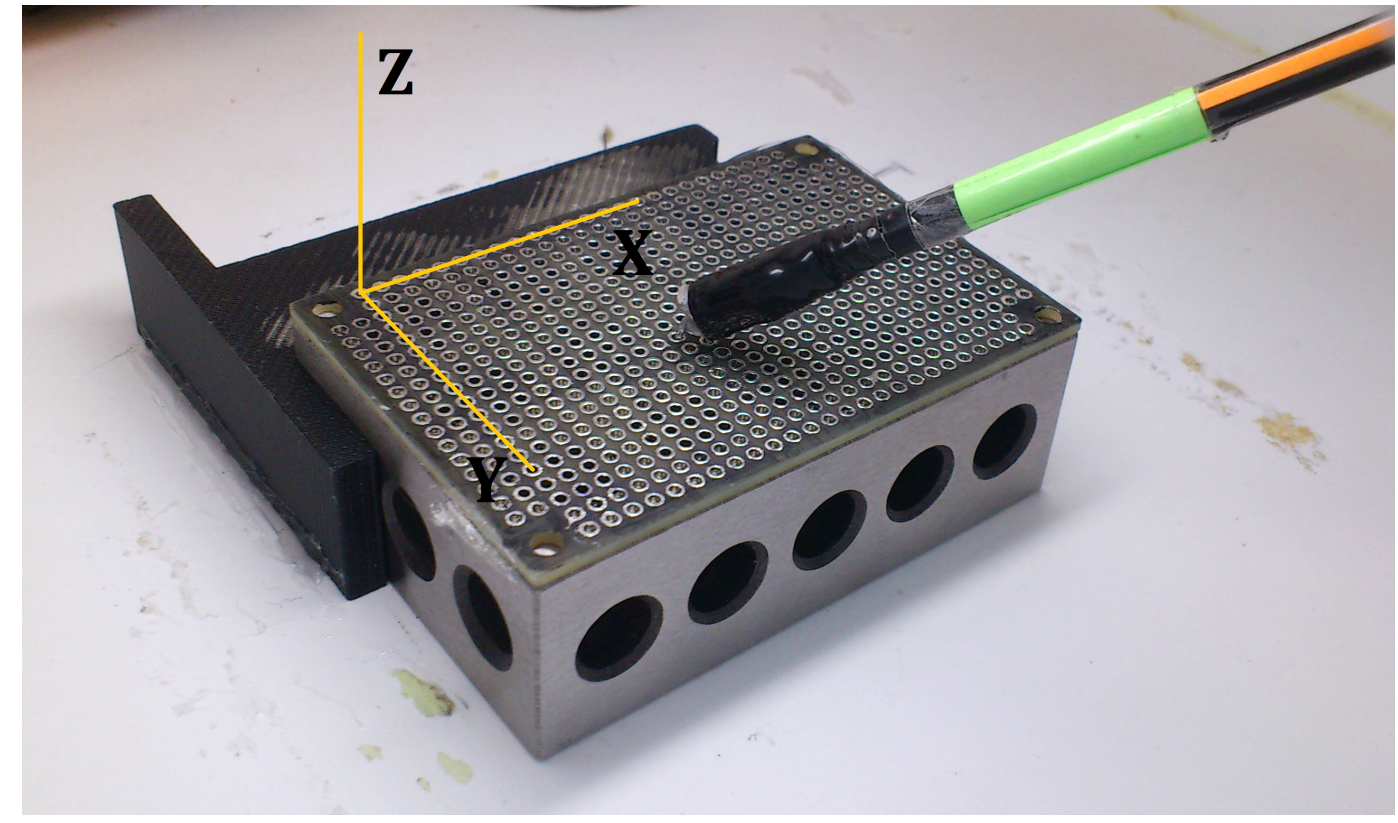

Figure 4.1: Instrument tip and calibration grid inside of the training box.

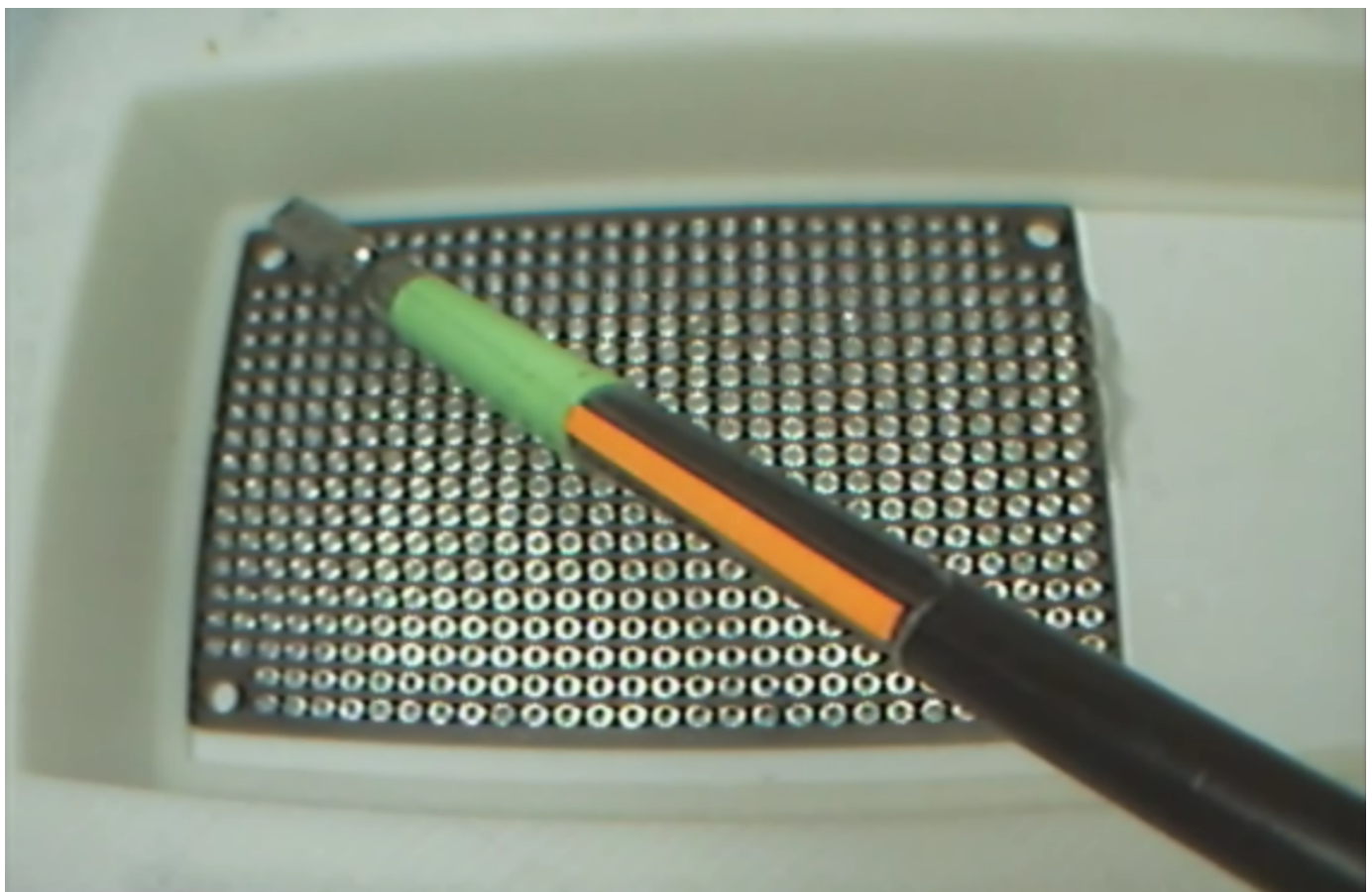

Figure 4.2: Sample image captured from the laparoscopic camera during a 20-point test. 
holds for electromagnetic tracking systems that are sensitive to perturbations of the generated electromagnetic field.

Since system testing occurred over the span of several months in a dynamic lab environment, it became standard procedure to register both systems prior to each data collection trial. Furthermore, each registration was confined to points within the volume of that test. Registration using data within a 20 -point volume would be more localized than registration over a 60 -point volume. It was expected that data fit to fewer points would result in higher local accuracy when compared with a system registered across a larger volume, where global accuracy would improve at the cost of local accuracy. This methodology matches the registration process of most tracking systems; calibration must be performed prior to use and the best registration is one that uses points across the expected working volume of the procedure.

When the IRES tracker is first turned on, the device must be held stationary for a few seconds while the IMU corrects for sensor offsets and performs an internal magnetometer calibration. Similarly, the Ascension microBIRD 6-DOF electromagnetic tracking system requires up to 60 seconds after power up for output readings to stabilize.

A more rigorous magnetometer calibration routine, designed to correct for hard and soft iron distortions, is also recommended if the IRES tracker has been mounted to a new instrument. To calibrate for hard and soft iron effects, one method requires that the magnetometer be rotated to build a sphere of measurements as shown in Figure 4.3. Correction parameters are then determined and applied to fix sphere offset (hard iron effects) and deformation (soft iron effects). In Figure 4.3, the blue sphere represents uncalibrated data and the green sphere is corrected data. This calibration routine allows for accurate magnetic field sensing even in the presence of ferromagnetic material or permanent magnets attached to the instrument body.

\subsubsection{Testing Procedure}

After registration and calibration of the tracking system, the test procedure involves placing the instrument tip at each location on the absolute reference plane and holding it for a few seconds allowing 50-150 position estimates to be recorded. Keyboard input is used to signal when the instrument is being held at each location to facilitate post-capture analysis. Once the desired point 


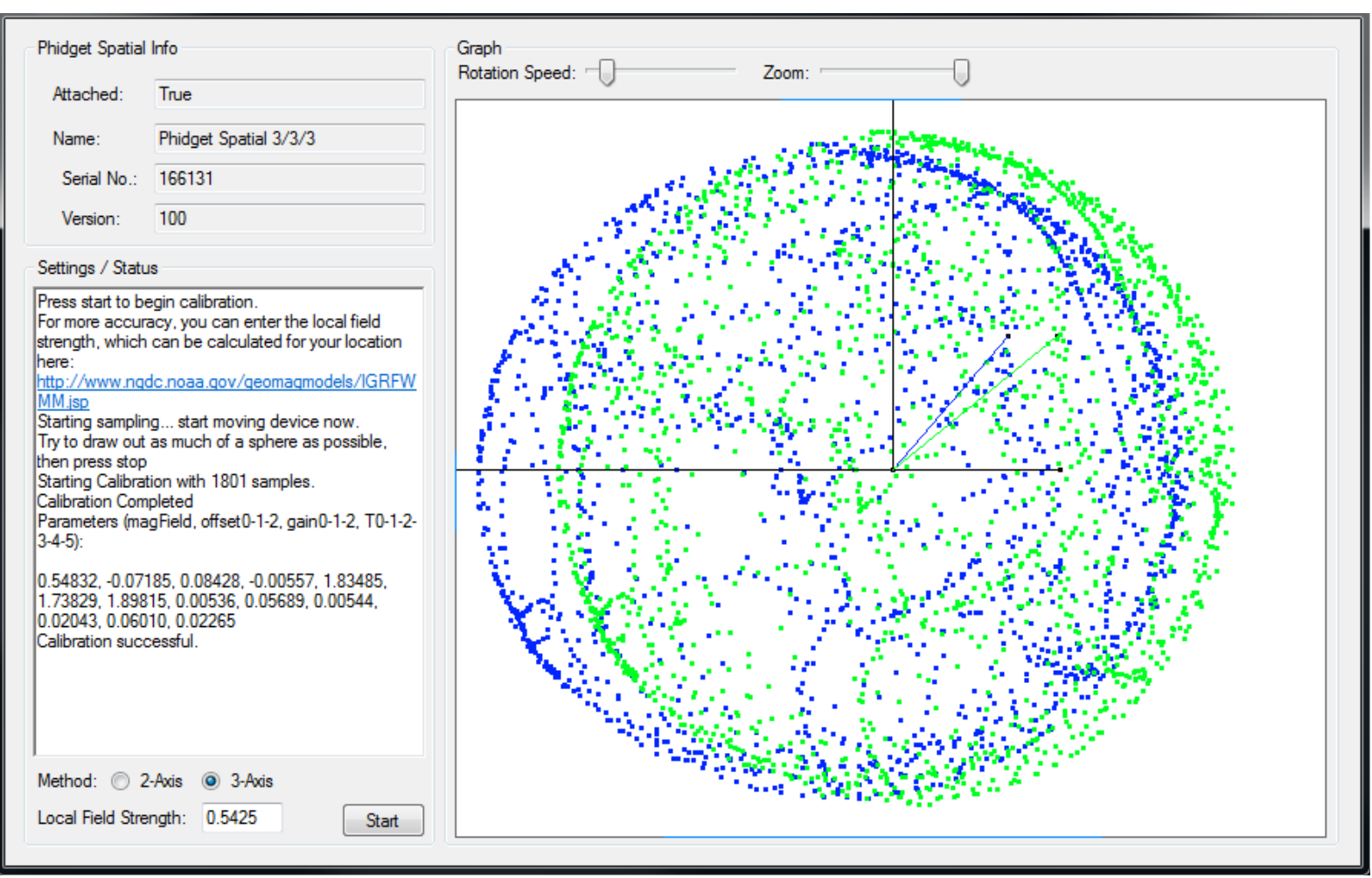

Figure 4.3: Magnetometer calibration for hard and soft iron field distortions.

volume has been captured using the blocks to raise the reference plane by $1^{\prime \prime}(25.4 \mathrm{~mm})$ and $2^{\prime \prime}$ $(50.8 \mathrm{~mm})$ in the case of 40 and 60 point volumes, a log file is saved and the system may be turned off. Since data analysis relies on ordered sets of points, it is important that the instrument tip follow the same pattern when capturing data.

In addition to testing the IRES and electromagnetic trackers using 20, 40 and 60 point volumes, the electromagnetic tracker introduces time-varying magnetic fields into the sensing environment, allowing for a third scenario to be examined. The three system scenarios are:

1. IRES Tracker: In this case, the IRES tracker operates in a magnetically stable environment with the electromagnetic tracker disabled. Any magnetic interference is due to nearby lab equipment. This represents a realistic test scenario.

2. IRES Tracker (with interference): In this case, the IRES tracker operates within the working volume of an operational electromagnetic tracker. Field oscillations are present and system accuracy is expected to degrade. This case is designed to test the magnetic interference 
rejection capabilities of the Kalman filter, as well as quantify system performance under a worst-case scenario.

3. Electromagnetic Tracker: In this case, the electromagnetic tracker operates normally. Since the IRES tracker does not affect electromagnetic tracking performance, it is disabled only as a precaution. This provides a baseline for comparison and analysis between these two systems.

\subsubsection{Accuracy}

Accuracy is a measure of trueness. An accurate 6-DOF instrument tracking system can be expected to provide the correct tip location and orientation after registration to the working frame. If the mean of a distribution of position estimates is close to the true location, the system can be considered accurate even if the distribution has high variance.

The testing methodology employed in this research provides a very thorough investigation of system accuracy. Data captured by either system at each point within the 20-, 40- and 60-point volumes is a distribution of position estimates. Thus, for a 60-point test, 60 estimates of system accuracy across a large working volume can be obtained for comparison with the absolute positional reference. Root mean square error (RMSE), also known as standard error, is a statistical measure of the magnitude of the error between the sensed position and true position and can be calculated as the square root of the variance. Equation 4.1 calculates RMSE for $n$ data points, where the true value is represented by $\hat{x}_{i}$ and each measured sample is $x_{i}$.

$$
\mathrm{RMSE}=\sqrt{\frac{1}{n} \sum_{i=1}^{n}\left(\hat{x}_{i}-x_{i}\right)^{2}}
$$

For points in 3-D Cartesian coordinates, the magnitude of the difference between the estimated and true values was used in place of $\left(\hat{x}_{i}-x_{i}\right)$, such that RMSE is a measure of the standard distance error between the point sets. 


\subsubsection{Orientation Accuracy}

This investigation does not focus on orientation-specific tests because position estimates of the IRES tracker are dependent on orientation estimates. If the IRES tracker displays good positional accuracy, orientation accuracy must also be good.

Due to the Kalman filtering approach, orientation error will most likely be introduced by accelerometer and magnetometer offsets which skew orientation measurements. Since roll and pitch estimates are both calculated from a single 3-axis accelerometer, it can be expected that the magnitude of sensor offset resulting in orientation error across dozens of trials will be similar for both axes. A testing approach that examines pitch and yaw error will be sufficient to quantify orientation accuracy of the IRES tracker.

As shown in Equations 3.38, 3.39 and 3.40 of Section 3.3.5, IRES position estimates are generated from pitch and yaw angles so positional error can be used to determine orientation error as shown in Equations 4.2 and 4.3 or 4.4, where $l_{\mathrm{rp} \mid \mathrm{ti}}$ is the distance from the pivot to instrument tip:

$$
\begin{gathered}
E \phi_{\mathrm{pitch}}=\sin ^{-1}\left(\frac{E_{\mathrm{z}}}{l_{\mathrm{rp} \mid \mathrm{ti}}}\right) \\
E \psi_{\text {yaw }}=\sin ^{-1}\left(\frac{E_{\mathrm{x}}}{l_{\mathrm{rp} \mid \mathrm{ti}} \cdot \cos \left(\phi_{\mathrm{pitch}}\right)}\right), \\
E \psi_{\text {yaw }}=\cos ^{-1}\left(\frac{E_{\mathrm{y}}}{-l_{\mathrm{rp} \mid \mathrm{ti}} \cdot \cos \left(\phi_{\mathrm{pitch}}\right)}\right) .
\end{gathered}
$$

This method relies on the assumption that all positional error is due to orientation error. Since the silicone pivot is free to deform, this condition cannot be guaranteed and points where force is exerted on the pivot must be excluded from this analysis. This can be achieved through observation of force applied at the instrument tip to hold it at a reference point. If any force is required, a reaction force exists at the pivot which may cause deformation and this point must be 
excluded from the dataset.

\subsubsection{Precision}

Precision is a measure of the quality of data as it relates to exactness. In the case of positional tracking, a precise system would display a narrow spread of estimated values, whereas an imprecise system would exhibit a much wider spread. A system can be precise but if the mean of the data values does not closely correlate with the true value, the system can still be inaccurate.

The precision of both the IRES and electromagnetic trackers can be quantified as the spread of captured points at any absolute reference location. For a 60-point volume, this means that each position distribution can be used to determine variance. The square root of the average variance across the working volume, also known as standard deviation, was calculated using Equation 4.5, where $x_{i}$ is each sample in the set and $\bar{x}$ is the mean of this sample distribution given by Equation 4.6:

$$
\begin{gathered}
\sigma=\sqrt{\frac{1}{n} \sum_{i=1}^{n}\left(x_{i}-\bar{x}\right)^{2}}, \\
\bar{x}=\frac{1}{n} \sum_{i=1}^{n} x_{i} .
\end{gathered}
$$

\subsubsection{Repeatability}

Repeatability is an indication of measurement stability across successive runs. A tracking system that is repeatable will give the same position estimate when returned to the same location across a number of trials.

Repeatability was assessed by performing the 20-, 40- and 60-point tests across several days with numerous implementations of the registration process and comparing the standard deviation at each point. If, for example, five trials were performed using the IRES tracker where a 60-point volume was captured, the means of each position distribution were used to determine the standard deviation at this point. Averaging these 60 standard deviations gives a global indication of the positional repeatability of the system. 


\subsubsection{Data Spread}

Quantifying accuracy by using RMSE is an easy way to present and compare results, but this approach obfuscates the true data spread from maximum to minimum value. Since these systems both operate at a fast refresh rate, a single outlying position estimate isn't very meaningful, so rather than report the 100th percentile, the 95th and 99th percentiles will be reported. The increase in spread from $95 \%$ to $99 \%$ provides a further measure of the magnitude and frequency of outlying values.

To quantify data spread, each position measurement from every test at each location in the volume was pooled and the scalar distance from this point to its corresponding absolute reference was determined. These distances were then ordered and the 95th and 99th percentile values were reported. Approximately 1.2 million data points were used to determine these percentile values and no data was omitted.

\subsection{Results}

Summary results are presented in Table 4.1. Provided as a visual aid, Figure 4.4 presents a 60-point test captured by the IRES tracker. Pre-registered points are on the left and registered IRES tracking output is on the right. The regular green grid pattern indicates absolute reference locations.

\subsubsection{Discussion}

The following discussion provides a detailed analysis of the data presented in Table 4.1.

\subsubsection{Accuracy}

The most notable result is that the IRES tracking system, free from electromagnetic tracker interference, displays an accuracy that is equal to, and better than, the electromagnetic tracker. For the 20- and 40-point volumes, system accuracy is virtually identical, with improved accuracy going to the IRES tracker as the volume increases to 60 points. As discussed in Section 4.1.1, these results also show the effect of a system registration process that is dependent on the size of 
Table 4.1: Summary of Results.

\begin{tabular}{|l|l|l|l|l|l|l|}
\hline 20-Point Volume & $\begin{array}{l}\text { Number } \\
\text { of Trials }\end{array}$ & $\begin{array}{l}\text { Accuracy } \\
(\text { RMSE })(\mathrm{mm})\end{array}$ & $\begin{array}{l}\text { Precision } \\
(\sigma)(\mathrm{mm})\end{array}$ & $\begin{array}{l}\text { Repeatability } \\
(\sigma)(\mathrm{mm})\end{array}$ & $\begin{array}{l}95 \% \\
(\mathrm{~mm})\end{array}$ & $\begin{array}{l}99 \% \\
(\mathrm{~mm})\end{array}$ \\
\hline IRES Tracker & 6 & 1.25 & 0.37 & 0.57 & 3.18 & 3.90 \\
\hline $\begin{array}{l}\text { IRES Tracker (with } \\
\text { interference) }\end{array}$ & 10 & 1.63 & 0.70 & 0.92 & 4.23 & 5.26 \\
\hline $\begin{array}{l}\text { microBIRD 6-DOF } \\
\text { Magnetic Tracker }\end{array}$ & 5 & 1.25 & 0.87 & 0.77 & 2.19 & 3.10 \\
\hline \hline 40-Point Volume & $\begin{array}{l}\text { Number } \\
\text { of Trials }\end{array}$ & $\begin{array}{l}\text { Accuracy } \\
(\text { RMSE) }(\mathrm{mm})\end{array}$ & $\begin{array}{l}\text { Precision } \\
(\sigma)(\mathrm{mm})\end{array}$ & $\begin{array}{l}\text { Repeatability } \\
(\sigma)(\mathrm{mm})\end{array}$ & $\begin{array}{l}95 \% \\
(\mathrm{~mm})\end{array}$ & $\begin{array}{l}99 \% \\
(\mathrm{~mm})\end{array}$ \\
\hline IRES Tracker & 6 & 1.74 & 0.38 & 0.79 & 4.89 & 5.75 \\
\hline $\begin{array}{l}\text { IRES Tracker (with } \\
\text { interference) }\end{array}$ & 7 & 2.02 & 0.74 & 0.99 & 5.95 & 7.47 \\
\hline $\begin{array}{l}\text { microBIRD 6-DOF } \\
\text { Magnetic Tracker }\end{array}$ & 2 & 1.73 & 0.93 & 0.73 & 4.01 & 4.63 \\
\hline \hline 60-Point Volume & $\begin{array}{l}\text { Number } \\
\text { of Trials }\end{array}$ & $\begin{array}{l}\text { Accuracy } \\
(\mathrm{RMSE})(\mathrm{mm})\end{array}$ & $\begin{array}{l}\text { Precision } \\
(\sigma)(\mathrm{mm})\end{array}$ & $\begin{array}{l}\text { Repeatability } \\
(\sigma)(\mathrm{mm})\end{array}$ & $\begin{array}{l}95 \% \\
(\mathrm{~mm})\end{array}$ & $\begin{array}{l}99 \% \\
(\mathrm{~mm})\end{array}$ \\
\hline IRES Tracker & 6 & 2.09 & 0.41 & 0.84 & 6.21 & 7.37 \\
\hline $\begin{array}{l}\text { IRES Tracker (with } \\
\text { interference) }\end{array}$ & 7 & 2.30 & 0.75 & 1.07 & 6.40 & 8.27 \\
\hline $\begin{array}{l}\text { microBIRD 6-DOF } \\
\text { Magnetic Tracker }\end{array}$ & 2 & 3.29 & 0.97 & 0.75 & 8.47 & 9.25 \\
\hline
\end{tabular}
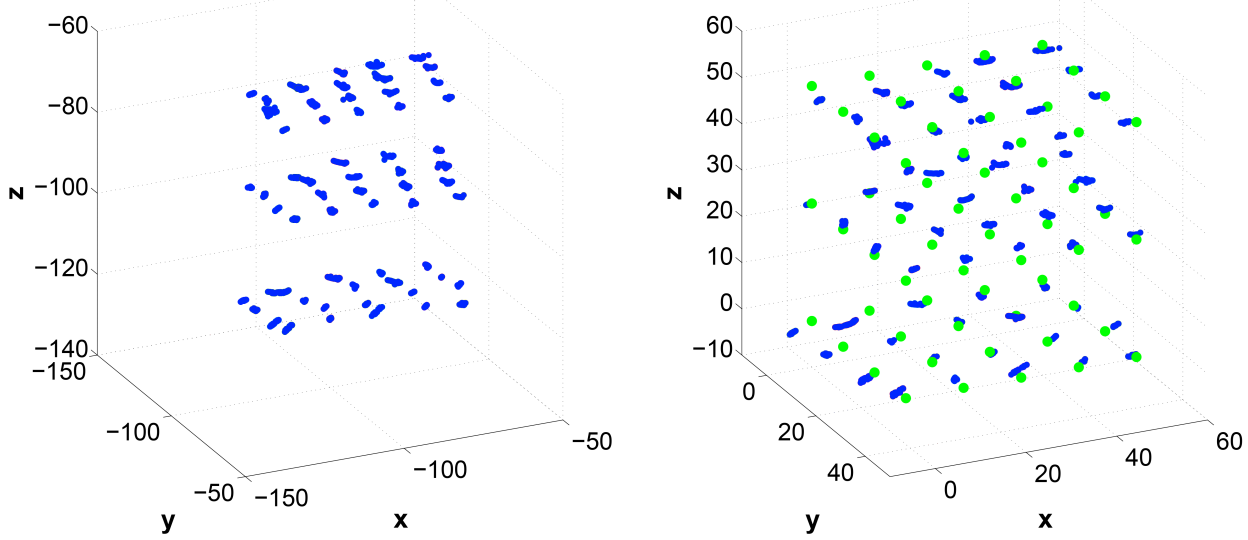

True Position

Measured

Position

Figure 4.4: Raw IRES tracker position information (left) and registered IRES tracker position information (right). 
the working volume. Calibration of both systems within a small working volume results in higher accuracy than can be achieved by calibration across a larger volume with more points.

Figure 4.5 shows position estimates for the IRES tracker in the XY-plane of a 20-point volume and Figure 4.6 shows an equivalent view of 20 points captured by the magnetic tracker. From these two figures it can be seen that the IRES tracker is able to generate more precise position estimates though both systems perform with the same accuracy. The increase in precision makes position error more visible.

From Table 4.1, it can be seen that accuracy of the electromagnetic tracker within a 60point volume is significantly worse than any other case. To investigate this loss of accuracy, a comparison of IRES and electromagnetic tracking data is provided in Figures 4.7 and 4.8. These figures present raw position estimates on the left and a side profile of calibrated position estimates in the XZ-plane on the right. From Figure 4.8, it is clear that the electromagnetic tracker is not providing good position estimates at $\mathrm{Z}=2^{\prime \prime}(\mathrm{Z}=50.8 \mathrm{~mm})$ as this plane of data appears shifted. A thorough investigation of this behaviour led to the conclusion that the steel blocks used to raise the reference grid were causing magnetic field distortions and degrading electromagnetic tracking performance. Steps were taken to mount the electromagnetic tracking probe as far away from these blocks as possible (380 $\mathrm{mm}$ from the instrument tip) and since this behaviour is not readily apparent with a single steel block, it must be assumed that the distortion effect is cumulative. Since registration of the electromagnetic tracking system included these shifted points, the bestfit solution resulted in a rotation of the volume and these results are not truly representative of electromagnetic tracking performance across a 60-point volume. Since the goal of this research is to test and validate the IRES tracker, it was decided that modification of the experimental design to improve electromagnetic tracking accuracy for this specific case was not necessary.

The behaviour of the electromagnetic tracker with the introduction of ferromagnetic material highlights a very important distinction between this system and the IRES tracker. The IRES tracker used the same steel blocks to capture points at $\mathrm{Z}=1^{\prime \prime}(\mathrm{Z}=25.4 \mathrm{~mm})$ and $\mathrm{Z}=2^{\prime \prime}$ ( $\mathrm{Z}$ $=50.8 \mathrm{~mm}$ ) and the IMU was mounted on the instrument $360 \mathrm{~mm}$ from the tip, yet unlike the electromagnetic tracker, the IRES tracker did not experience noticeable performance degradation. Since the IRES tracker derives a second orientation vector from magnetic field direction and this 


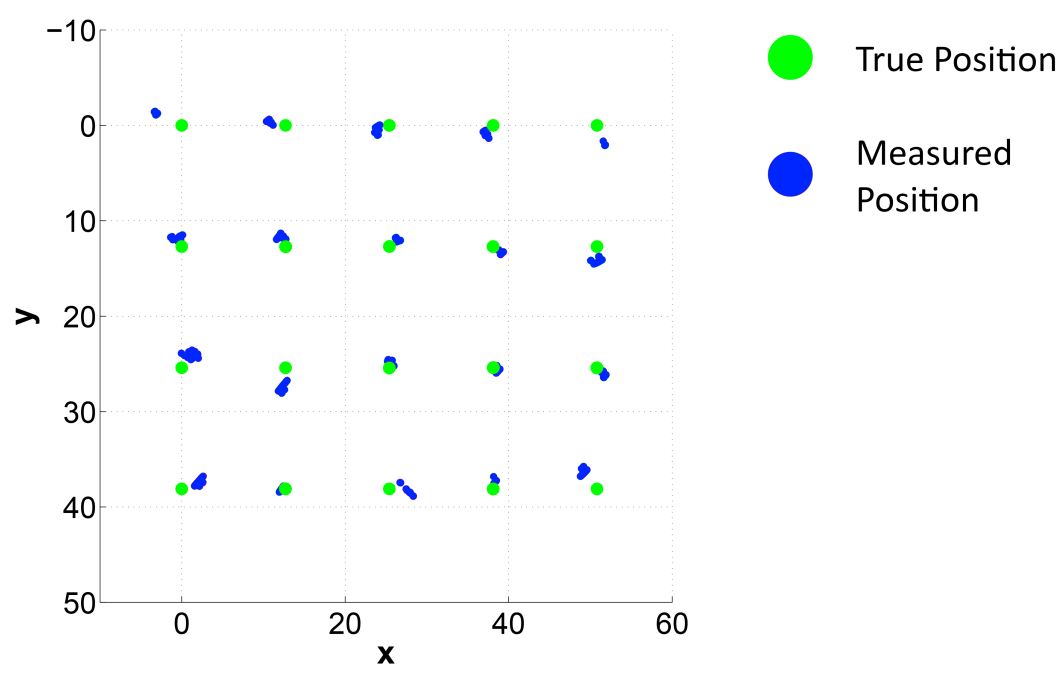

Figure 4.5: IRES tracker position estimates for a typical 20-point volume.

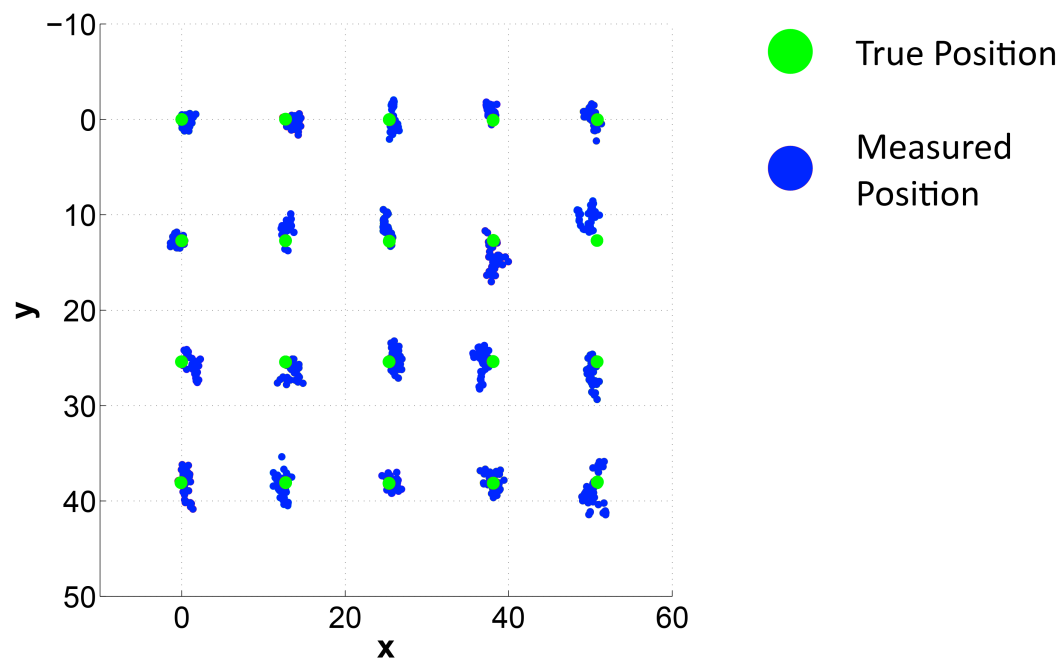

Figure 4.6: Electromagnetic tracker position estimates for a typical 20-point volume.

magnetic field is the sum of all local field effects, the introduction of small amounts of ferromagnetic material do not appear to perturb the local field to a significant degree. By contrast, the electromagnetic tracker creates a very specific field from which 6-DOF pose is derived and perturbations of this field will have a much greater effect on system accuracy. Since tip location estimates for the electromagnetic tracker rely on both orientation and position information, field perturbations will introduce error into both estimates and have a compound effect on tracker accuracy. The IRES tracker appears to exhibit greater immunity to ferromagnetic material introduced into the working 

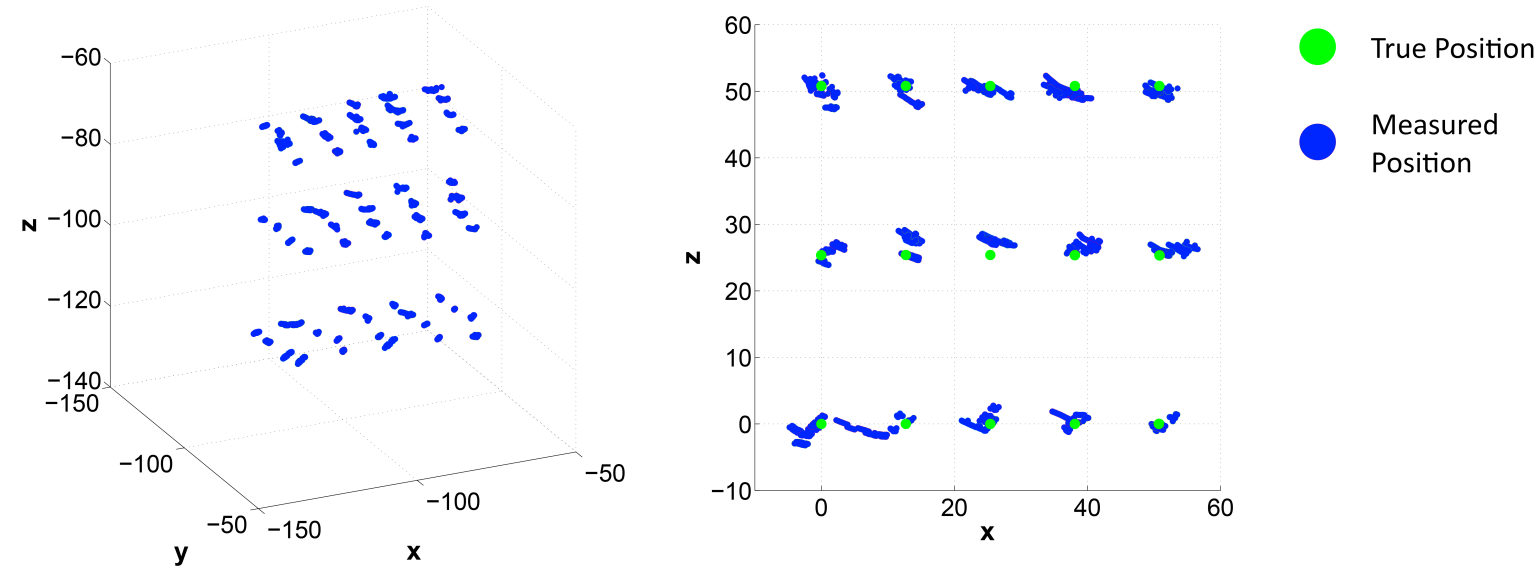

Figure 4.7: IRES tracker position estimates for a 60-point volume.
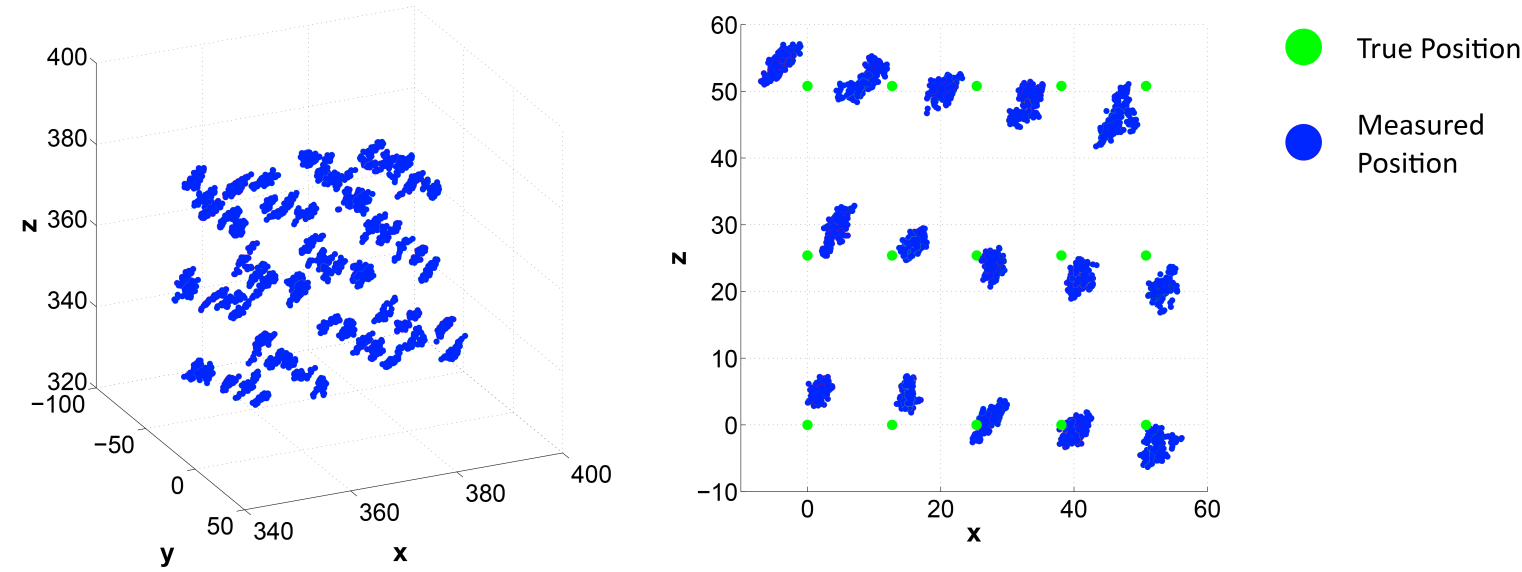

Figure 4.8: Electromagnetic tracker position estimates for a 60-point volume.

environment.

Continuing the investigation of magnetic interference on IRES tracking accuracy, Table 4.1 shows that performance degrades when the system is influenced by the time-varying magnetic field of an electromagnetic tracker. Figure 4.9 shows the effect of this magnetic interference on position estimates. In this figure, it can be seen that the point distributions trace out arcs, with a radial centre about the pivot point of the instrument. Since position information is projected from this datum, noisy orientation estimates along any single axis manifest as arc-like distributions. In this case, compassing performance has degraded, resulting in noisy yaw angle estimates. As expected, accuracy in the Z-direction remains unaffected since the magnetically-derived heading 
vector only affects estimates in the XY-plane.

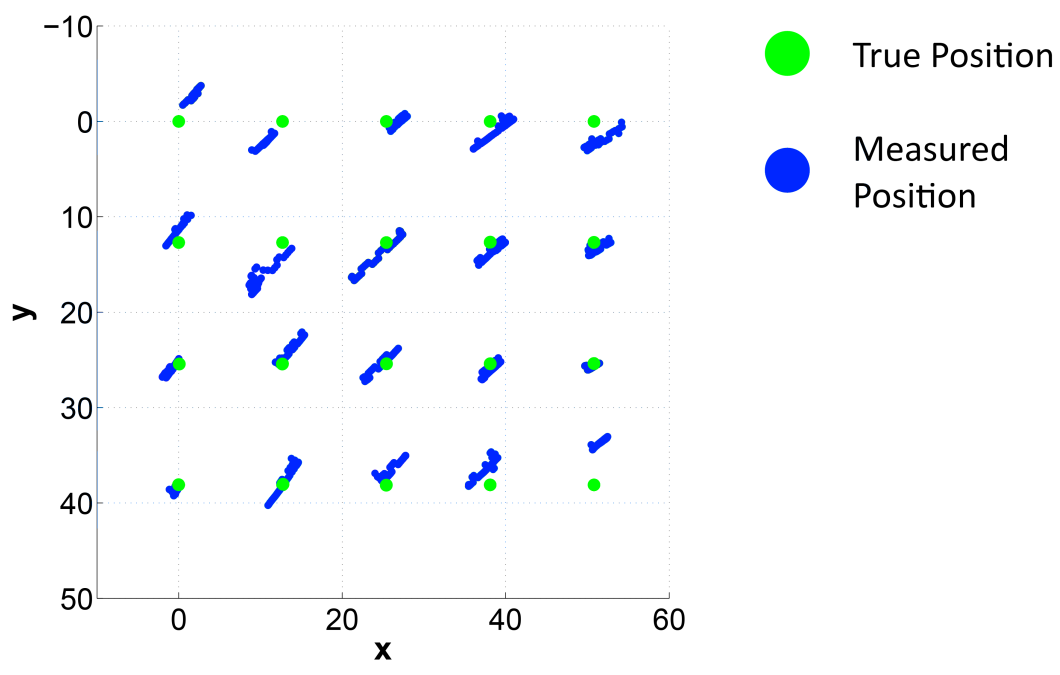

Figure 4.9: IRES tracker position estimates for a 20-point volume with time-varying electromagnetic interference.

While the IRES tracker is less accurate when operating within the working volume of an electromagnetic tracker, the system remains functional and continues to provide useful tracking information. This is largely the result of a well-tuned Kalman filter and proper definition of the $w_{\mathrm{R}}$ parameter used to scale the observation covariance matrix, as shown in Equation 3.32. Increasing $w_{\mathrm{R}}$ improves interference rejection, but overestimation will slow filter convergence and recovery from errors introduced by the process model. A well-tuned filter balances these two requirements for performance and robustness.

\subsubsection{Orientation Accuracy}

Table 4.2 presents orientation accuracy for the IRES tracker with and without electromagnetic tracker interference. Since roll and pitch errors are mainly due to offsets within the accelerometer, it can be expected that both axes will share similar performance. Furthermore, roll is not required for generating position estimates and need not be independently validated.

Following the test protocol outlined in Section 4.1.2.2 to ensure that no error was introduced by pivot deformation, it was found that exterior and corner points within the test volume exerted force on the instrument, with the greatest force being applied across the $\mathrm{Z}=2^{\prime \prime}(\mathrm{Z}=50.8 \mathrm{~mm})$ test 
Table 4.2: IRES Orientation Accuracy.

\begin{tabular}{|l|l|l|l|l|l|l|}
\hline & \multicolumn{3}{|c|}{ Pitch $\left(^{\circ}\right)$} & \multicolumn{3}{c|}{ Yaw $\left(^{\circ}\right)$} \\
\hline & RMSE & $95 \%$ & $99 \%$ & RMSE & $95 \%$ & $99 \%$ \\
\hline IRES Tracker & 0.38 & 0.74 & 0.93 & 0.58 & 1.10 & 1.52 \\
\hline $\begin{array}{l}\text { IRES Tracker (with } \\
\text { interference) }\end{array}$ & 0.38 & 0.76 & 0.94 & 0.73 & 1.42 & 1.82 \\
\hline
\end{tabular}

layer. These points were omitted, resulting in the use of 36 of 60 points for orientation analysis.

Table 4.2 shows that, as expected, yaw estimates are greatly affected by electromagnetic tracker interference, whereas pitch estimates are not. Even under the influence of electromagnetic interference, the RMSE and 99th percentile yaw errors remain well below the manufacturer-specified compass accuracy of $2^{\circ}$ from Table 3.4. This improvement in accuracy is largely due to the Kalman-based approach to sensor fusion.

\subsubsection{Precision}

Referring back to Table 4.1, the IRES tracker was considerably more precise than the electromagnetic tracker. This is due in large part to the stability and noise rejection performance of the tuned Kalman filter. As shown in Figure 4.9, variance in the orientation estimate results in a loss of precision. Table 4.3 shows IRES precision along each axis, measured as the standard deviation of each point distribution. From these results it can be seen that precision is independent of the size of the volume and that electromagnetic interference only degrades precision in the $\mathrm{X}$ and $\mathrm{Y}$ axes.

The precision of the electromagnetic tracker is not constant and tends to improve as the sensor probe is moved closer to the field generator. In these tests, the field generator was positioned directly beneath the table on which the training box was placed and, since the probe was well within the tracker's operating volume, these results can be considered to reflect a typical usage scenario. Even so, it is interesting to note that for those tests performed under the influence of electromagnetic interference, the IRES tracker retained a level of precision that is better than that seen by the electromagnetic tracker. 
Table 4.3: Detailed Precision Results.

\begin{tabular}{|l|l|l|l|}
\hline 20-Point Volume & X-Axis $(\sigma)(\mathrm{mm})$ & Y-Axis $(\sigma)(\mathrm{mm})$ & Z-Axis $(\sigma)(\mathrm{mm})$ \\
\hline IRES Tracker & 0.49 & 0.39 & 0.24 \\
\hline $\begin{array}{l}\text { IRES Tracker (with } \\
\text { interference) }\end{array}$ & 1.01 & 0.83 & 0.27 \\
\hline $\begin{array}{l}\text { microBIRD 6-DOF } \\
\text { Magnetic Tracker }\end{array}$ & 0.61 & 1.03 & 0.98 \\
\hline \hline 40-Point Volume & X-Axis $(\sigma)(\mathrm{mm})$ & Y-Axis $(\sigma)(\mathrm{mm})$ & Z-Axis $(\sigma)(\mathrm{mm})$ \\
\hline IRES Tracker & 0.51 & 0.37 & 0.27 \\
\hline $\begin{array}{l}\text { IRES Tracker (with } \\
\text { interference) }\end{array}$ & 1.02 & 0.82 & 0.38 \\
\hline $\begin{array}{l}\text { microBIRD 6-DOF } \\
\text { Magnetic Tracker }\end{array}$ & 0.66 & 1.10 & 1.04 \\
\hline \hline 60-Point Volume & $\mathrm{X}-\mathrm{Axis}(\sigma)(\mathrm{mm})$ & Y-Axis $(\sigma)(\mathrm{mm})$ & $\mathrm{Z}-\mathrm{Axis}(\sigma)(\mathrm{mm})$ \\
\hline IRES Tracker & 0.55 & 0.40 & 0.29 \\
\hline $\begin{array}{l}\text { IRES Tracker (with } \\
\text { interference) }\end{array}$ & 1.02 & 0.82 & 0.41 \\
\hline $\begin{array}{l}\text { microBIRD 6-DOF } \\
\text { Magnetic Tracker }\end{array}$ & 0.76 & 1.14 & 1.01 \\
\hline
\end{tabular}

\subsubsection{Repeatability}

System repeatability is the variation in repeated measurements from the tracking system under the same conditions. In a true repeatability test, system calibration and registration should not be changed, meaning that samples captured for the precision tests also provide a measure of repeatability. A more meaningful measure of repeatability known as reproducibility involves testing the system across several days under different conditions and calibrations. The trials presented in Table 4.1 spanned several months with each trial representing a complete test procedure involving initialization, instrument calibration and registration before data capture could be performed. As a result, the repeatability data presented herein is a combination of repeatability and reproducibility criteria, designed to gauge system reliability under real world conditions.

The 20-point tests of the IRES tracker display the highest level of repeatability, compared to all other test cases. Since the calibration process can more accurately register a smaller volume with fewer points, it was expected that the variation between successive runs within this volume would be lower than for larger volumes. Repeatability of the IRES tracker in the presence of 
electromagnetic interference was also degraded since the system is both less accurate and less precise.

By contrast, the electromagnetic tracking system displays the same repeatability performance regardless of the size of the test volume. This indicates that the loss of accuracy as the volume size increases to 60 points is a systematic error due to the introduction of the steel blocks. Since the electromagnetic tracker was only used as a reference system for comparison to the IRES tracker, only two trials were performed for the 40- and 60-point volumes. With two trials, only two mean positions were available to assess repeatability at any single location within the volume. The average of 60 repeatability values from these two sets is not a very strong measure of system repeatability and these results should be interpreted accordingly.

\subsubsection{95th and 99th Percentile}

The percentile values presented in Table 4.1 indicate the maximum distance error from the absolute position within which $95 \%$ and $99 \%$ of the captured data lies. For the IRES tracker in a 20-point volume, 1 in 20 samples may have a distance error greater than $3.18 \mathrm{~mm}$ but only 1 in 100 samples will have a distance error greater than $3.90 \mathrm{~mm}$. Presenting both percentile values shows that the system is not prone to spurious outliers and position estimates are well contained within a local region about each test point.

The electromagnetic tracker displays consistently tighter absolute data spread compared with the IRES tracker, if results for the 60-point tests are ignored for the reasons discussed in Section 4.2.1.1. Since the IRES tracker is more precise than the electromagnetic tracker, this distance spread must be due to shifting of the point distribution at each location. A distribution offset will result in higher data spread since each point is compared to the absolute positional reference. This shifting is likely due to orientation error and/or deformation of the silicone pivot point.

The 100th percentile was not reported as this value is not a good measure of system performance. The longer either system is left capturing data, the greater the probability of recording a more extreme outlying value. Power supply spikes and rogue EM interference are some sources of this random error and cannot be controlled. 


\subsubsection{Computational Performance}

MATLAB only natively supports single-threaded execution, limiting performance to a single CPU core and, though MATLAB is a powerful mathematical language for rapid code generation, studies have shown that $\mathrm{C}++$ equivalent approaches are capable of algorithm execution speeds in excess of 500 times faster [46]. MATLAB was selected due to the iterative nature of algorithm development and specialized functions not natively supported in other programming languages. Performance figures presented in this discussion represent final MATLAB algorithm implementation.

Table 4.4 shows computational load for the IRES tracker running on two different systems. In each case, only a single core could be tasked and though the IMU is capable of sampling at up to 250 samples per second, limitations inherent to using serial communication prevented rates from exceeding 58.8 SPS. Even at these sampling rates, the IRES tracker provided very fluid, real-time position and orientation estimates with minimal computational load.

Table 4.4: Computational Requirements for the MATLAB-based IRES Tracker.

\begin{tabular}{|l|l|l|}
\hline & $\begin{array}{l}\text { Intel Core i5 750 } \\
\text { @ 2.66 GHz }\end{array}$ & $\begin{array}{l}\text { Intel Core 2 Duo } \\
\text { SU7300 @ 1.3 GHz }\end{array}$ \\
\hline CPU Cores Available & 4 & 2 \\
\hline Cores Used & 1 & 1 \\
\hline Sampling Rate (SPS) & 58.8 & 58.8 \\
\hline Load Per Core & $4.40 \%$ & $6.92 \%$ \\
\hline
\end{tabular}

By eliminating the need for serial communication through the use of a faster protocol and by switching to a more efficient language, significant performance improvement can be expected. This opens up the potential for algorithm implementation on embedded devices.

\subsection{Summary}

In this chapter, performance of the IRES tracking system was assessed and compared to an electromagnetic tracking system. The testing methodology was presented and metrics such as accuracy, precision, repeatability and data spread were quantified and discussed.

Overall, performance of the IRES tracker was very similar to the electromagnetic tracking 
system with both trackers displaying similar accuracy and repeatability. Precision of the IRES tracker was twice that of the electromagnetic system though the latter was capable of a slightly narrower data spread. By subjecting the IRES tracker to time-varying electromagnetic fields and introducing ferromagnetic material into the sensing environment, this system was found to display superior magnetic interference rejection capability compared with the electromagnetic tracking system. Based on these results, the IRES tracking system provides a viable alternative to EM technology.

In the following chapter, a summary of the work and contributions made by this thesis is presented. The thesis is concluded and recommendations are made discussing areas of improvement and directions for future work. 


\section{Chapter 5}

\section{Conclusions and Recommendations}

This thesis presented a novel laparoscopic instrument tracking system based on the fusion of inertial, magnetic and distance sensing modalities. Designed to provide an alternate approach to popular optical and EM tracking technologies, the following research and design objectives were considered:

1. To develop a novel, noncontact laparoscopic instrument tracking system based on the fusion of existing technologies.

2. To provide real-time instrument position and orientation estimates.

3. To demonstrate interference rejection capabilities and/or provide continuous motion tracking under conditions that cause alternative technologies to fail.

4. To design an adaptable solution that can be used with a wide range of laparoscopic equipment.

5. To develop efficient algorithms that can be implemented on embedded processors.

6. To provide a cost-effective approach to laparoscopic motion tracking. This will reduce the barrier to entry over standard tracking systems. 
Through the development of the IRES tracker, each objective was considered and implemented, resulting in a tracking solution that largely achieved the project goals. A complete 6-DOF tracking solution was created through the development of lightweight algorithms to handle the fusion of multiple sensing modalities. The system demonstrated real-time operation with tracking performance that often met and exceeded the capabilities of an EM tracker under ideal and non-ideal conditions. The hardware package was designed with the ability to swap between a range of laparoscopic instruments at a cost of several hundred dollars compared with the cost for competing systems that often extends into five figures.

\subsection{Summary and Conclusions}

The IRES tracking system is a complete 6-DOF tracking solution for MIS laparoscopic instruments. The system uses inertial, magnetic and distance sensing modalities to compute orientation and instrument tip location based on established geometric relationships. Operation of the IRES tracker relies on the assumption that a point exists at the trocar-tissue interface where the instrument may pivot but where translatory motion is minimized. Any deflection of the trocar pivot point will cause elastic tissue deformation that tends to return the trocar to a location of minimal energy. This assumption was tested through the use of an MIS training box with silicone inserts that simulate elastic tissue. While a positive correlation was observed between pivot deformation and position error, these effects only became apparent at the outermost regions of larger test volumes that far exceed the field of view of a laparoscopic camera. Within typical working volumes for MIS procedures, pivot deformation was negligible and tracking performance was found to be similar to commercially available EM tracking solutions.

The IRES tracker is built upon the concept of an attitude-heading reference system (AHRS), where orientation angles can be determined from the fusion of accelerometer, gyroscope and magnetometer data. The sensor fusion algorithm employs an adaptation of the linear Kalman filter for tracking quaternion-based orientation. Since the Kalman filter is able to adapt to changes in signal variance, it can generate a more accurate solution compared with fixed-gain filters such as the complementary filter. The use of quaternions ensures continuous orientation tracking and 
avoids gimbal lock inherent to Euler-based methods.

With an accurate and robust orientation estimate, the trocar pivot point can be used as a datum from which positional information can be generated. An IR sensor measures the axial distance along the instrument from the sensor package to trocar allowing in-patient depth to be determined. Finally, orientation and depth information can be used to project the instrument tip location into Cartesian coordinates and provide position data.

The IRES tracker was evaluated using a set of calibration blocks to provide an absolute position reference and was also compared to the microBIRD 6-DOF EM tracking system by Ascension Technologies. Key metrics such as accuracy, precision, repeatability and data spread were used to gauge the performance of both systems and several special-case situations involving magnetic and ferromagnetic interference were investigated. It was found that position error for both systems was nearly identical, with the IRES tracker capable of a precision up to twice that of the EM tracker. Both systems proved to be highly repeatable with the IRES tracker showing greater immunity to ferromagnetic material introduced into the sensing environment. Although accuracy was slightly reduced, the IRES tracker was even capable of operating within the fluctuating magnetic environment of an EM tracker.

In conclusion, the IRES tracker is capable of excellent 6-DOF tracking with performance that is competitive with current-generation MIS laparoscopic tracking techniques at a small fraction of the cost. An IRES tracking system would be a good candidate for use in surgical training or a clinical environment.

\subsection{Recommendations}

The current version of the IRES tracker is a prototype, and as such, there are many ways in which the design can be improved for performance and functionality.

Embedded Processing: To ease algorithm development and refinement, all signal processing for the IRES system is currently performed on a desktop lab computer. Since the final algorithms are not computationally demanding, they could be ported to an embedded microcontroller such as Atmel AVR or PIC. This would alleviate much of the communication bandwidth between 
the IRES sensors and computer as only orientation and position would need to be relayed. Furthermore, a protocol could be developed to allow modification of the tracking algorithm with new filter tuning parameters, updated instrument specifications or even expanded functionality in future releases. A simple API could allow the IRES tracker to be easily interfaced with a wide range of development and commercial software.

Size and Packaging: Though the sensor package has been mounted to minimize interference to the surgeon and procedure, the IMU and IR sensors are relatively bulky. These devices can be replaced by smaller components to minimize the visual profile provided care is taken to maintain the required IMU sensor specifications. It would also be advisable to enclose the sensors in a case to protect exposed circuitry and make the system easier to clean.

Mount and Configuration: The current sensor package has been glued to the laparoscopic instrument but a mounting system could be designed to allow the IRES tracker to be quickly and securely fastened to handles of varying size and contour. As a result, one IRES device could provide tracking for a wide range of laparoscopic instruments. Since the initial calibration procedure for a new IRES device automatically detects instrument length and IMU offset, the need for custom software profiles can be avoided.

Care must be taken to design a mount that aligns the distance sensor with the head of the trocar. Initial tests showed that the IR sensor could often track an unmodified trocar and it may be possible to develop a reliable method of distance sensing without the need for an optically reflective disc. Further testing should be conducted to determine whether reliable axial distance sensing can be achieved for existing trocar designs. A different range sensing modality may also provide superior performance.

Wireless: In its current form, the IRES tracker is not well suited for use in a clinical environment. Two cables for power and high bandwidth communication must be run between the sensors and a lab computer. This restricts the free range of motion of the instrument. With a power requirement of $75 \mathrm{~mA}$ at $5 \mathrm{~V}$, the IRES tracker could be well suited for conversion to wireless operation. An $850 \mathrm{mAh}$ lithium polymer battery with voltage converter and charging circuit would occupy the same footprint as the Phidgets 9-DOF IMU providing over 8 hours 
of continuous operation. By switching to a Class 2 Bluetooth wireless radio and removing the on-board USB controller, power consumption would increase to $85 \mathrm{~mA}$ allowing over 7 hours of continuous operation. When switching to a wireless communication protocol such as Bluetooth, care must be taken to ensure that no appreciable lag develops between the tracker and host computer.

Future Validation: Beyond technical design improvements, recommendations can be made for future work involving a more rigorous validation procedure. Since this tracking method relies so heavily on the assumption that tissue deformation only results in negligible translation of the trocar pivot point, this assertion must be more thoroughly examined. The validation provided in this thesis made use of soft silicone rubber to simulate elastic tissue properties but not all MIS surgical sites will react the same way. The current version of the IRES tracker would be well-suited for motion capture in a surgical training environment. Before this system can be considered for use in an operating environment, proper tissue deformation studies must be conducted. Validation of this system for use in surgical procedures is a far more involved process that could not be adequately covered within the scope of this work.

A more thorough validation of the IRES tracker for use in surgical training could also be conducted. By using a training box to simulate various MIS procedures, the operator could determine whether reliance on IRES pose information could be trusted to produce the desired result. A variety of silicone stiffnesses could also be molded and tested, corresponding to a broader range of tissue properties. Ultimately, the best validation procedure will involve the use of the IRES tracker in real-world procedures such as in-vivo trials. 


\section{References}

[1] C. Dougan, "US markets for laparoscopic devices 2013," Millennium Research Group, December, 2012. Available from: http://mrg.net/Products-and-Services/Reports.aspx.

[2] S. Yamaguchi, D. Yoshida, H. Kenmotsu, T. Yasunaga, K. Konishi, S. Ieiri, H. Nakashima, K. Tanoue, and M. Hashizume, "Objective assessment of laparoscopic suturing skills using a motion-tracking system," Surgical Endoscopy, vol. 25, no. 3, pp. 771-775, 2011.

[3] M. Chmarra, C. Grimbergen, and J. Dankelman, "Systems for tracking minimally invasive surgical instruments," Minimally Invasive Therapy \& Allied Technologies, vol. 16, no. 6, pp. 328 $340,2007$.

[4] E. Watanabe, T. Watanabe, S. Manaka, Y. Mayanagi, and K. Takakura, "Three-dimensional digitizer (Neuronavigator): New equipment for computed tomography-guided stereotaxic surgery," Surgical Neurology, vol. 27, pp. 543-547, July 1987.

[5] H. Reinhardt and H. Landolt, "CT-guided real time stereotaxy," in Advances in Stereotactic and Functional Neurosurgery 8, vol. 46 of Acta Neurochirurgica Supplementum, pp. 107-108, Springer Vienna, 1989.

[6] P. Doshi, L. Lemmieux, D. Fish, S. Shorvon, W. Harkness, and D. Thomas, "Frameless stereotaxy and interactive neurosurgery with the ISG Viewing Wand," in Advances in Stereotactic and Functional Neurosurgery 11, vol. 64 of Acta Neurochirurgica Supplementum, pp. 49-53, Springer Vienna, 1995.

[7] E. P. Sipos, S. A. Tebo, S. J. Zinreich, D. M. Long, and H. Brem, "In vivo accuracy testing and clinical experience with the ISG Viewing Wand," Neurosurgery, vol. 39, pp. 194-202, July 1996.

[8] F. Corazza, R. Stagni, V. Parenti Castelli, and A. Leardini, "Articular contact at the tibiotalar joint in passive flexion," Journal of Biomechanics, vol. 38, pp. 1205-1212, June 2005.

[9] Faro Technologies Inc., "Faro arm accuracy." Customer Service Department Technical Guides, January, 2013. Available from: ftp://ftp.faro.com/FastFaxes/06REF176-ArmFastFaxes/ 06REF176-003-FaroArmAccuracy. doc.

[10] Faro Technologies Inc., "FaroArm Fusion." Online, 2013. Available from: http://www.faro. com/products/metrology/faroarm/.

[11] Ascension Technology Corporation, "microBIRD," 2013. Available from: http://www . ascension-tech.com. 
[12] Polhemus, "FASTRAK," 2013. Available from: http://www.polhemus.com/?page=motion_ fastrak.

[13] NDI, "Aurora," 2013. Available from: http://www.ndigital.com/medical/aurora.php.

[14] Claron Technology Inc., "MicronTracker," 2013. Available from: http://www.clarontech. com/microntracker.php.

[15] NDI, "Polaris," 2013. Available from: http://www.ndigital.com/medical/ polarisfamily.php.

[16] O. Tonet, R. U. Thoranaghatte, G. Megali, and P. Dario, "Tracking endoscopic instruments without a localizer: A shape-analysis-based approach," Computer Aided Surgery, vol. 12, no. 1 , pp. 35-42, 2007.

[17] A. Trejos, A. Lyle, A. Escoto, M. Naish, and R. Patel, "Force/position-based modular system for minimally invasive surgery," in IEEE International Conference on Robotics and Automation (ICRA), (Anchorage, Alaska), pp. 3660-3665, May 3-8, 2010.

[18] D. Tarrant, C. Roberts, D. Jones, C. Yang, and C. Lin, "Rapid and robust transfer alignment," in IEEE 1st Regional Conference on Aerospace Control Systems, (Westlake Village, California), pp. 758-762, May 25-27, 1993.

[19] J. Collin, O. Mezentsev, and G. Lachapelle, "Indoor positioning system using accelerometry and high accuracy heading sensors," in Proceedings of the 16th International Technical Meeting of the Satellite Division of The Institute of Navigation (ION GPS/GNSS), (Portland, Oregon), pp. 1164-1170, Sept. 9-12, 2003.

[20] E. Nebot and H. Durrant-Whyte, "Initial calibration and alignment of low-cost inertial navigation units for land vehicle applications," Journal of Robotic Systems, vol. 16, pp. 81-92, Feb. 1999 .

[21] H. Ren and P. Kazanzides, "Hybrid attitude estimation for laparoscopic surgical tools: A preliminary study," in IEEE Annual International Conference of the Engineering in Medicine and Biology Society (EMBS), vol. 31, (Minneapolis, Minnesota), pp. 5583-5586, Sept. 2-6, 2009 .

[22] H. Ren, D. Rank, M. Merdes, J. Stallkamp, and P. Kazanzides, "Multisensor data fusion in an integrated tracking system for endoscopic surgery," IEEE Transactions on Information Technology in Biomedicine (T-ITB), vol. 16, pp. 106-111, Jan. 2012.

[23] H. Ren and P. Kazanzides, "Investigation of attitude tracking using an integrated inertial and magnetic navigation system for hand-held surgical instruments," IEEE/ASME Transactions on Mechatronics, vol. 17, pp. 210-217, Apr. 2012.

[24] J. Farrell and M. Barth, The Global Positioning System 83 Inertial Navigation. McGraw-Hill Companies, Incorporated, 1999.

[25] E. Asadi and M. Bozorg, "A decentralized architecture for simultaneous localization and mapping," IEEE/ASME Transactions on Mechatronics, vol. 14, pp. 64-71, Feb. 2009. 
[26] VectorNav, "VN-200 Rugged INS/GPS," 2012. Available from: http://www.vectornav . com/products/vn200-rug.

[27] SBG Systems, "IG-500N inertial navigation system with embedded GPS," March 2013. Available from: http://www.sbg-systems.com/products/ig500n-miniature-ins-gps.

[28] Sensonor, "STIM300 inertial measurement unit," May 2013. Available from: http://www . sensonor.com/gyro-products/inertial-measurement-units/stim300.aspx.

[29] Honeywell, "Integrated compass sensor HMC6052," 2013. Available from: http: //www51.honeywell.com/aero/common/documents/myaerospacecatalog-documents/ Missiles-Munitions/HMC6052.pdf.

[30] SHARP, "GP2D120 optoelectronic device," 2006. Available from: http://sharp-world. com/products/device/lineup/selection/index.html.

[31] S. O. Madgwick, "An efficient orientation filter for inertial and inertial/magnetic sensor arrays," Report, x-io Technologies Limited, April 30, 2010. Available from: http://www.x-io. co.uk/open-source-imu-and-ahrs-algorithms/.

[32] S. Madgwick, A. J. L. Harrison, and R. Vaidyanathan, "Estimation of IMU and MARG orientation using a gradient descent algorithm," in IEEE International Conference on Rehabilitation Robotics (ICORR), (Zurich, Switzerland), pp. 155-161, June 29-July 1, 2011.

[33] M. Cheguini and F. Ruiz, "Real-time attitude estimation based on gradient descent algorithm," in IEEE 4th Columbian Workshop on Circuits and Systems (CWCAS), (Barranquilla, Colombia), pp. 136-141, Nov. 1-2, 2012.

[34] J. Marins, X. Yun, E. Bachmann, R. Mcghee, and M. Zyda, "An extended Kalman filter for quaternion-based orientation estimation using MARG sensors," in IEEE/RSJ International Conference on Intelligent Robots and Systems (IROS), vol. 4, (Maui, Hawaii), pp. 2003-2011, Oct. 29-Nov. 3, 2001.

[35] X. Yun, E. Bachmann, and R. McGhee, "A simplified quaternion-based algorithm for orientation estimation from earth gravity and magnetic field measurements," IEEE Transactions on Instrumentation and Measurement, vol. 57, no. 3, pp. 638-650, 2008.

[36] D. Comotti and M. Ermidoro, "Report of the course "Progetto di microelettronica" - English reduced version," 2011. Available from: https://code.google.com/p/ 9dof-orientation-estimation/.

[37] R. E. Kalman, "A new approach to linear filtering and prediction problems," Transactions of the ASME - Journal of Basic Engineering, vol. 82, no. 1, pp. 35-45, 1960.

[38] S. Julier and J. Uhlmann, "Unscented filtering and nonlinear estimation," Proceedings of the IEEE, vol. 92, no. 3, pp. 401-422, 2004.

[39] N. El-Sheimy, E.-H. Shin, and X. Niu, "Kalman filter face-off: Extended vs. unscented Kalman filters for integrated GPS and MEMS inertial," Inside GNSS, pp. 48-54, March 2006. 
[40] E.-H. Shin and N. El-Sheimy, "An unscented Kalman filter for in-motion alignment of low-cost IMUs," in IEEE/ION Position Location and Navigation Symposium (PLANS), (Monterey, California), pp. 273-279, Apr. 26-29, 2004.

[41] M. S. Grewal, V. D. Henderson, and R. S. Miyasako, "Application of Kalman filtering to the calibration and alignment of inertial navigation systems," IEEE Transactions on Automatic Control, vol. 36, no. 1, pp. 3-13, 1991.

[42] J. L. Crassidis, K.-L. Lai, and R. R. Harman, "Real-time attitude-independent three-axis magnetometer calibration," Journal of Guidance, Control, and Dynamics, vol. 28, no. 1, pp. $115-120,2005$.

[43] S. Julier, "The spherical Simplex unscented transformation," in Proceedings of the American Control Conference (ACC), vol. 3, (Denver, Colorado), pp. 2430-2434, June 4-6, 2003.

[44] B. K. P. Horn, "Closed-form solution of absolute orientation using unit quaternions," Journal of the Optical Society of America A (JOSA A), vol. 4, no. 4, pp. 629-642, 1987.

[45] M. J, "Absolute orientation - Horn's method," September, 2010. Available from: http://www.mathworks.com/matlabcentral/fileexchange/ 26186-absolute-orientation-horns-method.

[46] T. Andrews, "Computation time comparison between MATLAB and C++ using launch windows," Aerospace Engineering, California Polytechnic State University, June, 2012. 


\section{VITA}

Name: $\quad$ Tara A. Bracken

Post-secondary The University of Western Ontario

Education and London, Ontario, Canada, 2009-2011, 2013

Degrees: $\quad$ M.E.Sc., Mechanical and Materials Engineering

The University of Western Ontario

London, Ontario, Canada, 2005-2009

B.E.Sc., Mechanical and Materials Engineering

Awards and Computer-Assisted Medical Intervention (CAMI) Scholarship

Scholarships: $\quad 2010-2011$

Related Work Teaching Assistant

Experience: (Instructor: Dr. Michael D. Naish)

MME 4452 - Robotics and Manufacturing Automation

MME 9610 - Applied Measurement and Sensing Systems

The University of Western Ontario

London, Ontario, Canada, 2010-2011

Graduate Research Assistant

(Supervisor: Dr. Michael D. Naish)

Canadian Surgical Technologies and Advanced Robotics (CSTAR)

The University of Western Ontario

London, Ontario, Canada, 2009-2011, 2013 